\title{
BISON TRISO Modeling Advancements and Validation to AGR-1 Data
}

September 2020

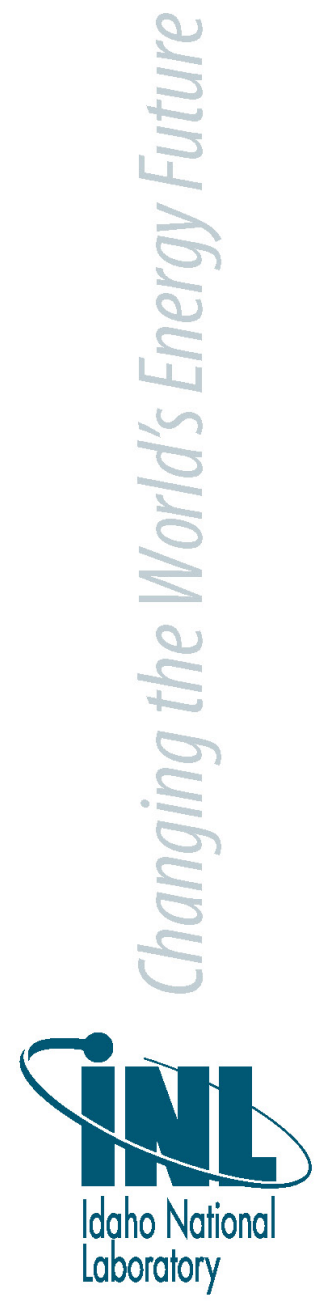

Jason D. Hales

Idaho National Laboratory

Wen Jiang

Idaho National Laboratory

Aysenur Toptan

Idaho National Laboratory

Kyle Gamble

Idaho National Laboratory 


\section{DISCLAIMER}

This information was prepared as an account of work sponsored by an agency of the U.S. Government. Neither the U.S. Government nor any agency thereof, nor any of their employees, makes any warranty, expressed or implied, or assumes any legal liability or responsibility for the accuracy, completeness, or usefulness, of any information, apparatus, product, or process disclosed, or represents that its use would not infringe privately owned rights. References herein to any specific commercial product, process, or service by trade name, trade mark, manufacturer, or otherwise, does not necessarily constitute or imply its endorsement, recommendation, or favoring by the U.S. Government or any agency thereof. The views and opinions of authors expressed herein do not necessarily state or reflect those of the U.S. Government or any agency thereof. 


\title{
BISON TRISO Modeling Advancements and Validation to AGR-1 Data
}

\author{
Jason D. Hales \\ Wen Jiang \\ Aysenur Toptan \\ Kyle Gamble
}

September 2020

Idaho National Laboratory

Computational Mechanics and Materials Department

Idaho Falls, Idaho 83415

http://www.inl.gov

Prepared for the

U.S. Department of Energy

Office of Nuclear Energy

Under DOE Idaho Operations Office

Contract DE-AC07-05ID14517 
Page intentionally left blank 


\begin{abstract}
BISON is a finite element-based nuclear fuel performance code. Among its unique characteristics are its ability to model 1D, 2D, and 3D geometries and its applicability to a wide variety of nuclear fuels. For the last eight years, BISON has included a beginning capability to model tri-structural isotropic (TRISO) fuel. Recently, interest in TRISO fuel has grown, and a significant effort has been made to improve BISON's capabilities in this area. Capability development has occurred for each material present in TRISO fuel particles: the buffer, inner pyrolytic carbon, silicon carbide, and outer pyrolytic carbon layers, as well as the fuel kernel. New elastic, creep, swelling, thermal expansion, thermal conductivity, and fission gas release (FGR) models are available. New models for the graphite matrix are also now available.
\end{abstract}

Another important addition is the ability to perform statistical failure analysis of large samples of fuel particles. This new capability, which continues to grow, enables evaluation of failure due to pressure or crack formation by analyzing many thousands of particles. This enables realistic calculations of fission product release from the many particles in a TRISO-fueled reactor.

These capabilities were checked via regression and verification tests. A large number of code benchmarking problems were also run, showing that BISON's results closely match those of other software tools. Finally, a significant validation effort was completed in which fission product release, measured as part of the AGR-1 capsule experiments, was compared to BISON outputs. BISON outputs compared very well to the experimental data and to PARFUME results.

Interest in BISON's TRISO capabilities is growing, with the U.S. Nuclear Regulatory Commission (NRC) and Westinghouse Electric Company receiving training during the past year. Multiple other entities have expressed interest in or are actively using BISON. Kairos Power, LLC, has a strong partnership with Idaho National Laboratory (INL) regarding the use of BISON for TRISO analysis.

While its capabilities still continue to grow, BISON has already become a powerful tool for TRISO analysis. 
Page intentionally left blank 


\section{Acknowledgments}

The partnership of Kairos Power, LLC, including contributions to the source code and documentation for TRISO capabilities, is gratefully acknowledged.

This work was funded by the U.S. Department of Energy (DOE)'s Nuclear Energy Advanced Modeling and Simulation (NEAMS) and Consortium for Advanced Simulation of Light Water Reactors (CASL) programs. The submitted manuscript has been authored by a contractor of the U.S. Government under Contract DE-AC07-05ID14517. Accordingly, the U.S. Government retains a non-exclusive, royalty-free license to publish or reproduce the published form of this contribution, or allow others to do so, for U.S. Government purposes. 
Page intentionally left blank 


\section{Contents}

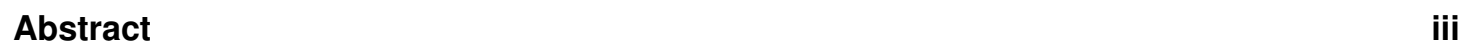

Acknowledgments $\quad$ v

List of Figures . . . . . . . . . . . . . . . . . . . . . . . . . . . . . . . .

List of Tables ......................... . . . . $\mathrm{x}$

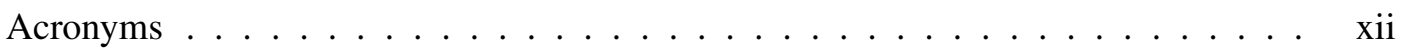

1 Introduction $\quad 1$

2 Collaboration with Kairos Power, LLC $\quad 4$

3 Development in BISON $\quad 6$

3.1 Mesh Generation . . . . . . . . . . . . . . . . . . . . . . 6

3.2 Material Models . . . . . . . . . . . . . . . . . 7

3.2.1 Thermal Properties ................... . . . 8

3.2.2 Mechanical Properties .................. 14

3.2.3 Fission Product Diffusion . . . . . . . . . . . . . . . . 25

3.2 .4 Fission Gas . . . . . . . . . . . . . . . . . 25

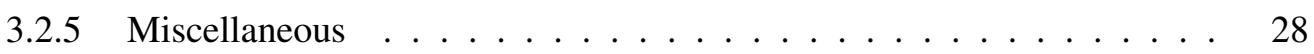

3.2.6 BISON Benchmarked against PARFUME . . . . . . . . . . . 31

3.3 Monte Carlo Evaluation and Statistical Failure Capabilities . . . . . . . . . . . 33

3.3.1 Failure Modes ................... 34

3.3.2 Weibull Failure Theory . . . . . . . . . . . . . . 35

3.3.3 High-Fidelity Analysis .................. 37

3.3.4 Preliminary Failure Probability Calculation . . . . . . . . . . 39

4 Verification $\quad \mathbf{4 1}$

4.1 Code Comparison: CRP-6 ..................... . . 41

4.1.1 Previously Run Fuel Performance Cases: 1-8, 10, 11 . . . . . . . 41

4.1.2 New Fuel Performance Cases: 9, 12,13 . . . . . . . . . . . . . . 44

4.1.3 New Diffusion Cases: 1-5 . . . . . . . . . . . . . . . . . 45

4.2 Code Comparison: AGR-5/6/7 Irradiation Test Predictions . . . . . . . . . . 48

4.3 Code Verification . . . . . . . . . . . . . . . . . . . 49 
5 Validation $\quad 59$

5.1 AGR-1 ........................ 59

5.1 Silver Release . . . . . . . . . . . . . . . . . 60

5.1.2 Cesium and Strontium Release . . . . . . . . . . . . . 61

6 Documentation and Training 66

6.1 Tutorial on TRISO Analysis in BISON . . . . . . . . . . . . . . . . . 67

6.2 TRISO Workshop Material . . . . . . . . . . . . . . 67

6.3 Recent Training Workshops . . . . . . . . . . . . . . . . 67

$\begin{array}{lll}7 & \text { Conclusions and Future Work } & 71\end{array}$

$\begin{array}{ll}\text { Bibliography } & 76\end{array}$ 


\section{List of Figures}

3.1 A simple one-dimensional mesh for a TRISO particle. Each material is represented by three finite elements. . . . . . . . . . . . . . . . . . .

3.2 A close-up view of a portion of a TRISO mesh where the kernel ends and the buffer begins. . . . . . . . . . . . . . . . . .

A two-dimensional mesh of a TRISO particle. This mesh may be created in BISON according to user preferences. . . . . . . . . . . . . . 8

3.4 The uranium oxicarbide (UCO) thermal properties. . . . . . . . . . . . . . 9

3.5 The buffer thermal conductivity. . . . . . . . . . . . . . . . 10

3.6 Monolithic silicon carbide $(\mathrm{SiC})$ thermal properties. . . . . . . . . . . . . . . 11

3.7 The graphite matrix thermal conductivity. . . . . . . . . . . . . . . 13

3.8 The graphite matrix specific heat capacity. . . . . . . . . . . . . . . . . . . . . . 14

3.9 UCO kernel elastic properties. . . . . . . . . . . . . . . . . . 15

3.10 The buffer mechanical properties. . . . . . . . . . . . . . . . 17

3.11 pyrolytic carbon $(\mathrm{PyC})$ elastic modulus. . . . . . . . . . . . . . . . . . 18

$3.12 \mathrm{PyC}$ creep coefficient. . . . . . . . . . . . . . . . . . . . . . . . . . 19

$3.13 \mathrm{PyC}$ thermal expansion coefficients. . . . . . . . . . . . . 20

3.14 Buffer and PyC isotropic irradiation strain at $\rho_{0}=1.96 \mathrm{~g} / \mathrm{cm}^{3} \ldots \ldots \ldots 21$

$3.15 \mathrm{PyC}$ radial irradiation strain at $\rho_{0}=1.96 \mathrm{~g} / \mathrm{cm}^{3} \ldots \ldots \ldots 22$

3.16 PyC tangential irradiation strain at $\rho_{0}=1.96 \mathrm{~g} / \mathrm{cm}^{3} \ldots \ldots \ldots 23$

3.17 Comparison of code results. . . . . . . . . . . . . . . . 32

3.18 Tangential stress at the inner surface of the $\mathrm{SiC}$ layer. . . . . . . . . . . . 34

3.19 Cracking of inner $\mathrm{PyC}(\mathrm{IPyC}) \ldots \ldots \ldots \ldots \ldots$

3.20 Aspect ratio of an aspherical particle. . . . . . . . . . . . . 36

3.21 Aspherical Particle. . . . . . . . . . . . . . . . . . 36

3.22 High-fidelity analysis and stress correlation. . . . . . . . . . . . . . 37

3.23 Stress correlation for IPyC cracking. . . . . . . . . . . . . . . . . . . . . 38

3.24 Stress correlation for an aspherical particle. . . . . . . . . . . . . . . . 39

4.1 Comparison of code results for inner-wall tangential stress in IPyC and SiC . . . 43

4.2 Results for Cases 10 and 11. Both the original (BISON13) and current BISON results are within the range of results from other codes. . . . . . . . . . . . . 43

4.3 Results for Case 9. Results from other codes extracted from [1]. . . . . . . . . 44

4.4 Results for Case 12. Results from other codes extracted from [1]. . . . . . . . . 45

4.5 Results for Case 13. Results from other codes extracted from [1]. . . . . . . . . 45

4.6 Comparison of BISON and Particle Fuel Model (PARFUME) results for innerwall tangential stress in SiC . . . . . . . . . . . . . . . . 50 
4.7 Buffer outer and IPyC inner radii during irradiation. Gap is computed as their difference. . . . . . . . . . . . . . . . . 51

4.8 Temperature from the kernel centerline to the outer surface of the outer PyC

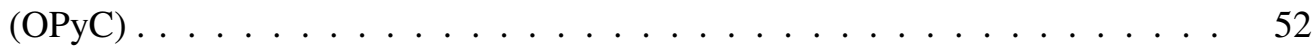

4.9 Evolution of $\mathrm{SiC}$ failure probability. . . . . . . . . . . . . . . 53

4.10 A pictorial representation of expected convergence behavior. . . . . . . . . . 56

4.11 BISON results for Problem 4.3.1 using one-dimensional finite elements. . . . . 57

4.12 BISON results for the Problem 4.3.2 using one-dimensional finite elements. . . 58

5.1 Comparison of measured and computed silver release fractions for seventeen

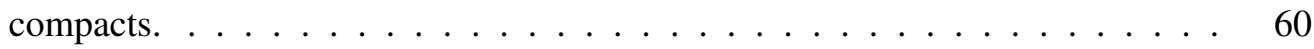

5.2 Concentration of silver in Compact 6-1-1 . . . . . . . . . . . . 61

5.3 Concentration of silver, cesium, and strontium in Compact 6-1-1. . . . . . . . 62

5.4 Comparison of measured and computed cesium release fractions for six compacts with no failed particles. . . . . . . . . . . . . . . . . . 63

5.5 Comparison of measured and computed cesium release fractions for three compacts with one or two failed particles. . . . . . . . . . . . . . 63

5.6 Comparison of measured and computed strontium release fractions for six compacts with no failed particles. . . . . . . . . . . . . . . . . . . 64

5.7 Comparison of measured and computed strontium release fractions for three compacts with one or two failed particles. . . . . . . . . . . . . 65

5.8 Concentrations of cesium and strontium for an intact and a failed particle in Compact 6-3-2. . . . . . . . . . . . . . . . . 65

6.1 BISON homepage. . . . . . . . . . . . . . . . . . . . 66

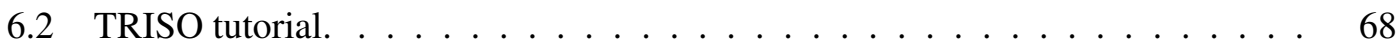

6.3 Discussion of the kernel and buffer layer at the TRISO tutorial page. . . . . . . 69

6.4 A working input file is part of the TRISO tutorial page. . . . . . . . . . . . 69

6.5 Representative slides from the TRISO workshop at https : / moosef ramework . org/bison_triso_workshop. . . . . . . . . . . . . . . . 70 


\section{List of Tables}

3.1 Coefficients for unirradiated thermal conductivity [2] . . . . . . . . . . . . 11

3.2 Isotropic strain at irradiation temperature of $1100^{\circ} \mathrm{C}$ and fast fluence of $3.7 \times 10^{25}$

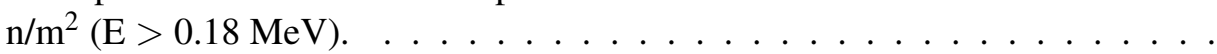

3.3 Isotropic strain and difference between radial and tangential strain at an irradiation temperature of $1100^{\circ} \mathrm{C}$ and fast fluence of $3.7 \times 10^{25} \mathrm{n} / \mathrm{m}^{2}(\mathrm{E}>0.18$

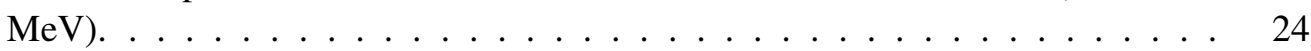

3.4 Elastic modulus of the $\mathrm{SiC}$ as a function of temperature. . . . . . . . . . . . 25

3.5 Mass diffusion coefficients. . . . . . . . . . . . . . . . 25

3.6 Ranges of $\mathrm{Kr}$ and $\mathrm{Xe}$ in $\mathrm{U}, \mathrm{C}$, and $\mathrm{O}$ [3] . . . . . . . . . . . . . . 26

3.7 The theoretical densities of the UCO mixture components. . . . . . . . . . . . . 29

3.8 The theoretical densities of the UCO mixture components. . . . . . . . . . 30

3.9 Bacon anisotropy factor (BAF) vs. fast neutron fluence $(\mathrm{E}>0.18 \mathrm{MeV}) \ldots \ldots$

3.10 Irradiation conditions. . . . . . . . . . . . . . . . 33

3.11 Fuel parameters. . . . . . . . . . . . . . . . . . 33

3.12 Statistical variation of fuel parameters. . . . . . . . . . . . . . . 40

3.13 Stress correlation factors and effective mean strength. . . . . . . . . . . . . 40

3.14 Failure probability. . . . . . . . . . . . . . . . . . . . 40

4.1 Comparison of analytical solution values for maximum tangential stress (MPa) to original (BISON13) and current BISON results for Cases 1-3 . . . . . . . .

4.2 Comparison of the range of values reported by Coordinated Research Program (CRP-6) participants to original (BISON13) and current BISON results for Cases $4 \mathrm{a}-7 \ldots \ldots \ldots \ldots \ldots \ldots \ldots \ldots \ldots \ldots \ldots$

4.3 Comparison of the range of values reported by CRP-6 participants to BISON results for Case 1, fractional release of cesium from a bare kernel. . . . . . . .

4.4 Comparison of the range of values reported by CRP-6 participants to BISON results for Case 2, fractional release of cesium from the kernel, buffer, and inner PyC . . . . . . . . . . . . . . . . . . .

4.5 Comparison of the range of values reported by CRP-6 participants to BISON results for Case 3, fractional release of cesium from the TRISO particle. . . . .

4.6 Comparison of the range of values reported by CRP-6 participants to BISON results for Case 4, fractional release of cesium and silver from the TRISO particle with 500 days of irradiation.

4.7 Comparison of the range of values reported by CRP-6 participants to BISON results for Case 5, fractional release of cesium and silver from TRISO particle with 10 cycles of irradiation. 
4.8 Fuel parameters. . . . . . . . . . . . . . . . . . . . . . . . . . . . . . 49

4.9 Irradiation conditions. . . . . . . . . . . . . . . . . . . . 54

4.10 Stress correlation factors and effective mean strength . . . . . . . . . . . . . 54

4.11 Failure probability. . . . . . . . . . . . . . . . . . . 54 


\section{Acronyms}

AGR

BAF

CASL

CRP-6

DOE

FE

FGR

FIMA

HTGR

IAEA

INL

IPyC

LTE

LWR

MES

MMS

MOOSE

NEAMS

NRC

OPyC

PARFUME

PDE

PIE

PyC

SiC

TRISO

UCO
Advanced Gas Reactor

Bacon anisotropy factor

Consortium for Advanced Simulation of Light Water Reactors

Coordinated Research Program

U.S. Department of Energy

finite element

fission gas release

fissions per initial metal atom

high-temperature gas reactor

International Atomic Energy Agency

Idaho National Laboratory

inner PyC

linear truncation error

light-water reactor

method of exact solutions

method of manufactured solutions

Multiphysics Object-Oriented Simulation Environment

Nuclear Energy Advanced Modeling and Simulation

U.S. Nuclear Regulatory Commission

outer PyC

Particle Fuel Model

partial differential equation

post-irradiation examination

pyrolytic carbon

silicon carbide

tri-structural isotropic

uranium oxicarbide 
Page intentionally left blank 


\section{Introduction}

BISON is a nuclear fuel performance tool. It is based on the finite element (FE) method and relies on the Multiphysics Object-Oriented Simulation Environment (MOOSE) [4]. One of the advantages of BISON is its ability to model a variety of fuel forms. Historically, fuel performance codes were built to model a specific fuel type. FRAPCON [5], FRAPTRAN [6], FALCON [7], and TRANSURANUS [8] are fuel performance codes for light-water reactors (LWRs). LIFEMETAL [9], SESAME [10], and MACSIS [11] are for metallic nuclear fuel. PARFUME [12], TIMCOAT [13], STRESS3 [14], and ATLAS [15] are fuel performance codes for tri-structural isotropic (TRISO) fuels. BISON is applicable to all three fuel forms, as well as plate fuels and others.

Hand in hand with BISON's applicability to model multiple fuel forms is its ability to accept a wide variety of geometries. Spherically symmetric geometry is most common for TRISO fuels, while axisymmetric geometry is most often used when modeling LWR and metallic fuel. BISON handles these common configurations but is also able to accept any geometry that can be represented by FEs. In the specific case of TRISO fuel, spherically symmetric models, axisymmetric models, and models with symmetry across Cartesian planes all have their place, for example when modeling defects or failures.

Another of BISON's advantages is its ability to run either very small or very large analyses. Very small problems might run in a few seconds on a single processor. Very large problems might run for a few hours on several processors on a cluster or supercomputer. Due to the capabilities BISON inherits from MOOSE, it is also possible to run many small problems on a cluster simultaneously, taking advantage of its many processors. This capability has proven to be essential for the statistical evaluation of failure of TRISO fuels in BISON.

BISON is built to adapt and grow. Distinct interfaces for material models, boundary conditions, output options, etc., have allowed many dozens of scientists to contribute to the code. These researchers come from U.S. Department of Energy (DOE) national laboratories, academia, and industry. Flexibility in fuel type, geometry, and problem size, in combination with the ease with which it may be customized and enhanced, has made BISON an integral part of the Advanced Fuels Campaign, Consortium for Advanced Simulation of Light Water Reactors (CASL), Nuclear Energy Advanced Modeling and Simulation (NEAMS), and multiple DOE-sponsored and university-led research projects. Increasingly, BISON is being used by industry for LWR and advanced reactor fuel analyses.

Given the relative abundance of available experimental data on LWR fuel rods, early development in BISON focused on LWR fuel [16]. However, even in its first few years of existence, BISON's potential for modeling TRISO fuel in a novel way was clear. 
Nominally, TRISO fuel is spherically symmetric. Using this symmetry, the governing equations of heat transfer, fission product transfer, and stress may be simplified greatly. This fact is leveraged by tools such as PARFUME and enables quick analyses of standard particles. Although TRISO particles fail infrequently, they do fail, and the failures occur in ways that are not spherically symmetric. In order for a code restricted to spherical symmetry to calculate failure information, another tool is needed. In the case of PARFUME, multidimensional, FE calculations from Abaqus [17] are required to give insight into failure behavior [12, 18].

In contrast, BISON's inherent ability to model a variety of geometries gives it the potential to model TRISO fuel in all situations, including particles with manufacturing defects.

As early as 2010, near the beginning of BISON development, the BISON team at INL began modeling TRISO fuel. In 2011 and 2012, the team pushed forward a set of TRISO models sufficient to evaluate a broad set of standard and customized scenarios [19]. The TRISO capabilities were based on the three partial differential equations at the center of all fuel performance modeling activities: energy balance (Eq. 1.1), conservation of momentum or static equilibrium (Eq. 1.2), and mass diffusion (Eq. 1.3).

The heat equation is:

$$
\rho C_{p} \frac{\partial T}{\partial t}+\nabla \cdot \mathbf{q}-E_{f} \dot{F}=0
$$

where $T$ is temperature, the primary unknown. $\rho$ and $C_{p}$ are density and specific heat, $\mathbf{q}$ is the heat flux, and $E_{f}$ is the energy released per fission. $\dot{F}$ is the volumetric fission rate.

Conservation of momentum, or static equilibrium, is prescribed using Cauchy's equation:

$$
\nabla \cdot \boldsymbol{\sigma}+\rho \mathbf{f}=0
$$

where $\boldsymbol{\sigma}$, the Cauchy stress tensor, is a function of displacement, the primary variable. $\mathbf{f}$ is the body force per unit mass (e.g., gravity).

Fission product species conservation, or mass diffusion, is given by:

$$
\frac{\partial C}{\partial t}+\nabla \cdot \mathbf{J}+\lambda C-S=0
$$

where $C$ is concentration, the primary variable. $\lambda$ and $S$ are the radioactive decay constant and source rate of a given species, and $\mathbf{J}$ is the mass flux.

The fundamental capabilities needed to analyze TRISO fuel were already available due to work on LWR fuel. Material models specific to the buffer, pyrolytic carbon, and silicon carbide layers were added, along with a handful of other behavioral models specific to TRISO fuel. Details can be found in [19].

Considerable verification and benchmarking were undertaken in [19]. Much of that relied upon a Coordinated Research Program (CRP-6) through the International Atomic Energy Agency 
(IAEA). A handful of simple problems with analytic solutions, along with a few more complicated problems without analytic solutions but with computed solutions supplied by CRP-6 participants, were used to demonstrate the correctness of BISON's predictions. Details are found in Chapter 4, which also includes results from additional CRP-6 cases.

Very little TRISO development occurred in BISON between the publication of [19] up until 2019. The capability was presented at multiple DOE-sponsored events and included in training material during that period. However, the capability did not generate significant interest.

In 2019, as momentum grew in the advanced reactor sector, multiple institutions began to show interest in TRISO modeling. Kairos Power, LLC; General Atomics; Westinghouse Electric Company; and the NRC have all shown interest in TRISO fuel and BISON's ability to model it. This interest has driven much of the development outlined in subsequent chapters, with Chapter 2 highlighting one strong collaboration.

The remainder of this report outlines the new capabilities in BISON (see Chapter 3), the rigorous verification of those capabilities (see Chapter 4), and the validation of BISON's TRISO functionality to AGR-1 data (see Chapter 5). Chapter 6 focuses on available documentation and training specific to TRISO fuel analysis with BISON. 


\section{Collaboration with Kairos Power, LLC}

Kairos Power has been a very close collaborator on recent TRISO development in BISON, and their contributions deserve to be emphasized. Kairos's reactor concept involves fuel pebbles that house TRISO particles in a molten salt coolant. The fuel design is similar to that for hightemperature gas reactors (HTGRs), but there are important differences. For that reason, much of the experimental testing and computational evaluation of TRISO fuel done in support of HTGRs is relevant to Kairos's design. Where there are computational gaps to be filled, Kairos intends to use BISON.

One of Kairos's fuel engineers is a former INL employee. This helped foster a functioning collaboration between the two teams. In addition, NEAMS has emphasized building connections with the advanced reactor community, and NEAMS-led meetings and conversations between program and Kairos leadership were instrumental in building the collaboration.

A partitioning of responsibilities with respect to TRISO development and evaluation in BISON has helped maximize efficiency. As each group has worked on its own specific tasks, frequent status update meetings helped communicate questions and progress. Questions were freely asked back and forth between both groups. This collaboration remains strong.

Kairos personnel have, to date, provided significant contributions to the new TRISO capabilities in BISON. These include:

- Evaluating existing capabilities

- Providing a list of new features necessary for BISON to match PARFUME's feature set

- Developing and documenting a first draft of many of those features, particularly in the area of material models

- Evaluating the correctness of models through code review and execution

- Providing recommendations regarding validation activities

- Providing answers to questions of all kinds, from the original references for particular models, to typical modeling approaches using PARFUME, to sources of relevant documentation.

Contributions made by INL personnel over this period include:

- Polishing the models and documentation provided by Kairos

- Developing new internal meshing options (see Section 3.1) 
- Creating additional material models (see Section 3.2)

- Developing a capability for statistical failure analysis (see Section 3.3)

- Refreshing the previously-run CRP-6 cases (see Section 4.1.1)

- Performing additional verification studies (see Sections 4.1.2, 4.1.3, and 4.3)

- Performing validation (see Chapter 5)

- Creating documentation, training, and example problems (see Chapter 6).

In addition to the existing collaboration already mentioned, Kairos Power, INL, Los Alamos National Laboratory, and Argonne National Laboratory were awarded $\$ 10 \mathrm{M}$ by DOE to advance Kairos's reactor design. Kairos received $\$ 5 \mathrm{M}$, and INL received $\$ 2 \mathrm{M}$-of which half is for TRISO work in BISON. This works is expected to be concluded in FY22.

Major tasks to be undertaken at INL for this project include:

- Reviewing the literature to learn what approaches the U.S. and foreign groups have used to model TRISO particles and pebbles

- Determining the availability and quality of diffusion coefficients for significant fission products

- Developing efficient approaches for modeling fission product diffusion, including diffusion in pebbles

- Developing a 3D fission product diffusion capability that incorporates the effects of defective particles

- Exercising statistical evaluation capabilities with the aim of computing failure probability estimates for Kairos Power's fuel

- Validating BISON results against CRP-6 cases and AGR-1 and AGR-2 data.

Clearly, Kairos Power and the BISON team are involved in a close collaboration. Kairos's needs are driving INL BISON development, and the capabilities in BISON are a central part of Kairos's strategy. It is anticipated that this mutually advantageous relationship will continue many years into the future. 


\section{Development in BISON}

Considerable BISON TRISO capability development has occurred recently in response to the needs of the advanced reactor community. This chapter reviews that development and is partitioned into three major sections: mesh generation; fundamental material models, such as those needed for heat conduction and stress analysis; and statistical capabilities, including models that play an integral role in determining failure probabilities.

More complete descriptions of these capabilities may be found in the documentation contained in the BISON repository. See also Chapter 6.

\subsection{Mesh Generation}

Finite element (FE) software such as BISON solve partial differential equations over some physical domain. That domain is represented by finite elements. Each FE represents a piece of the overall physical domain and is defined by the location of nodes associated with it. Some elements share nodes with other elements, tying the FEs together in to a single representation of the domain.

Analysis of TRISO fuel particles typically assumes perfect spherical geometry. With that assumption, the domain can be represented by a line of one-dimensional FEs. Each material layer in the TRISO fuel particle is represented by a set of FEs grouped together and labeled so BISON can apply the appropriate material properties to the different layers. The schematic in Fig. 3.1 shows elements grouped according to the material they represent and the nodes that define them.

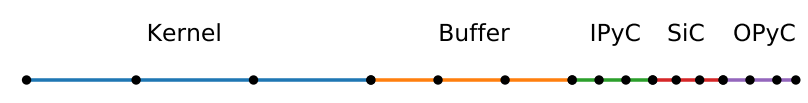

Figure 3.1: A simple one-dimensional mesh for a TRISO particle. Each material is represented by three finite elements.

Specialized software is often used to generate FE meshes. However, MOOSE has the ability to generate simple FE meshes, and that capability was extended and specialized for TRISO analysis. To use this capability, three main inputs are required for each of the kernel, buffer, inner 
pyrolytic carbon, silicon carbide, and outer pyrolytic carbon materials. These are the thickness (radius, for the kernel), the number of elements for each material, and a meshing bias.

The meshing bias allows elements to be concentrated toward one end of the material. The bias parameter $b$ represents the length of an element based on the length of the element to its left. For example, if $b=0.8$, the elements will get smaller from left to right, concentrating more elements at the right side of the material. If $b=1.5$, the elements will grow in size from left to right, with more, smaller elements at the left side of the material. An example of this bias feature is shown in Fig. 3.2. As can be seen, the FEs decrease in size in the kernel portion of the mesh and increase in size in the buffer portion. Biasing the mesh in this way is helpful when there are dramatic changes in diffusivity, as is the case with strontium diffusivity in the kernel and buffer. See Section 5.1.2 for more information.

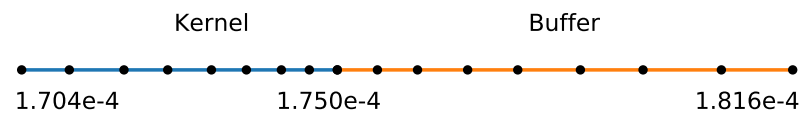

Figure 3.2: A close-up view of a portion of a TRISO mesh where the kernel ends and the buffer begins. The mesh becomes more refined toward the interface due to biasing. The coordinates on the figure are for the left-most node, the node at the interface of the kernel and the buffer, and the right-most node.

For failure analysis, two-dimensional and three-dimensional meshes are needed. Simple twodimensional meshes of TRISO particles can be built by BISON. For example, Fig. 3.3 shows a two-dimensional mesh of a quarter of a TRISO particle sectioned through its center. This mesh would be used in an axisymmetric analysis.

More complex two- or three-dimensional meshes require specialized software such as CUBIT [20].

\subsection{Material Models}

Thermal, mechanical, and mass diffusion models must be defined for each material, with some materials requiring more models than others. For example, some materials, though not all, require a creep model. Other models, such as those for FGR and burnup, are only defined for the fuel kernel. The material models described below are available in BISON as $\mathrm{C}++$ classes and are invoked through appropriate statements in the input file. Where the material model requires special code, the name of the code class is given in parentheses. Where the material model consists of simple constants for use in a standard model, no model name is given. 


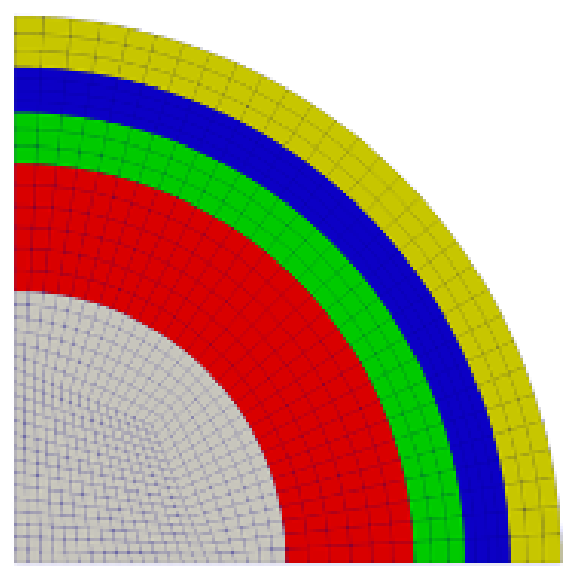

Figure 3.3: A two-dimensional mesh of a TRISO particle. This mesh may be created in BISON according to user preferences.

\subsubsection{Thermal Properties}

\subsubsection{UCO (UCOThermal)}

The thermal conductivity of UCO is given by [21]. Due to the lack of relevant UCO data, the functional relationship is derived from experimental data on $\mathrm{UO}_{2}$ and assumed valid for UCO. This relationship is assumed valid at all temperatures.

The thermal conductivity of the kernel, $k$ (W/m-K) (see Fig. 3.4), is given by:

$$
k=0.0132 \exp \left(0.00188 T_{C}\right)+ \begin{cases}\frac{4040}{464+T_{C}} & \text { for } T_{C}<1650^{\circ} \mathrm{C} \\ 1.9 & \text { for } T_{C} \geq 1650^{\circ} \mathrm{C}\end{cases}
$$

where $T_{C}\left({ }^{\circ} \mathrm{C}\right)$ is the temperature of the kernel. BISON uses and reports temperature in Kelvin. Conversions for this calculation are handled in the code.

The specific heat capacity of UCO is calculated from its molar heat capacity [22] and molar mass. Due to the lack of relevant UCO data, the functional relationship is derived from experimental data on $\mathrm{UO}_{2}$ and assumed valid for UCO.

$$
c_{P}=\frac{1}{M}\left(52.1743+87.951 t_{K}-84.2411 t_{K}^{2}+31.542 t_{K}^{3}-2.6334 t_{K}^{4}-\frac{0.71391}{t_{K}^{2}}\right)
$$

where $c_{P}(\mathrm{~J} / \mathrm{kg}-\mathrm{K})$ is the specific heat capacity, $t_{K}=T_{K} / 1000(\mathrm{~K})$ is the reduced temperature, and $M(\mathrm{~kg} / \mathrm{mol})$ is the molar mass (see Section 3.2.5). 


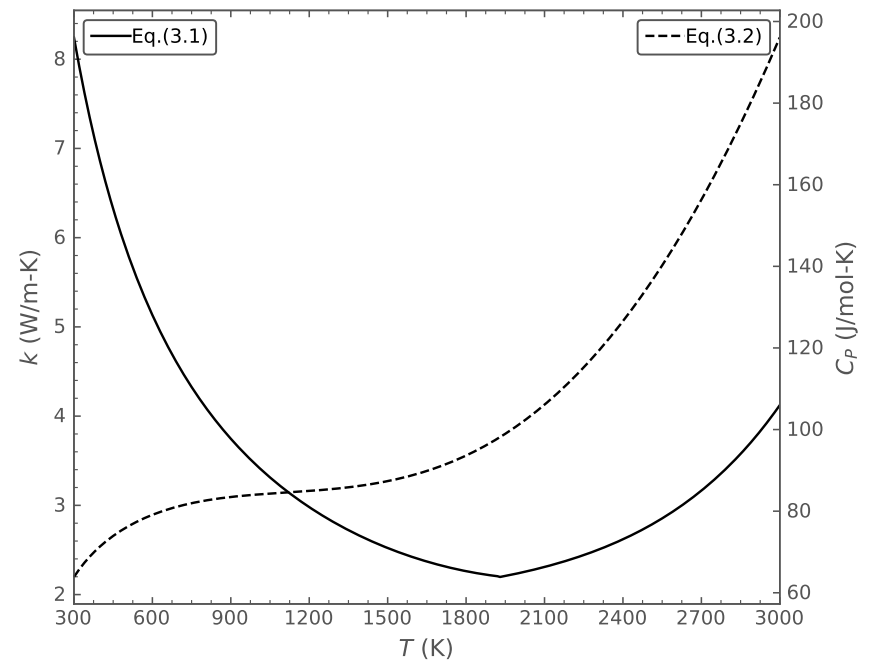

Figure 3.4: The UCO thermal properties.

\subsubsection{Buffer (BufferThermal)}

The thermal conductivity of the buffer is given by [23]:

$$
k=\frac{k_{\text {init }} k_{\text {theo }} \rho_{\text {theo }}\left(\rho_{\text {theo }}-\rho_{\text {init }}\right)}{k_{\text {theo }} \rho_{\text {theo }}\left(\rho_{\text {theo }}-\rho\right)+k_{\text {init }} \rho\left(\rho-\rho_{\text {init }}\right)},
$$

where $k(\mathrm{~W} / \mathrm{m}-\mathrm{K})$ is the thermal conductivity of the buffer and $\rho\left(\mathrm{kg} / \mathrm{m}^{3}\right)$ is the density of the buffer.

The values at initial $\left(\rho_{\text {init }}\right)$ and theoretical $\left(\rho_{\text {theo }}=2250 \mathrm{~kg} / \mathrm{m}^{3}\right)$ densities are taken from [24].

The initial thermal conductivity $\left(k_{\text {init }}\right)$ of the buffer is set to $0.5 \mathrm{~W} / \mathrm{m}-\mathrm{K}$. Though intended for an initial buffer density of $1000 \mathrm{~kg} / \mathrm{m}^{3}$, it is also used for slightly different densities $( \pm 10 \%)$. The thermal conductivity of the buffer at its theoretical density $\left(k_{\text {theo }}\right)$ is set to $4.0 \mathrm{~W} / \mathrm{m}-\mathrm{K}$.

The specific heat capacity of the buffer is given by [25] as $720 \mathrm{~J} / \mathrm{kg}-\mathrm{K}$.

\subsubsection{PyC}

The thermal conductivity of the PyC layers is $4 \mathrm{~W} / \mathrm{m}-\mathrm{K}$ [21].

The specific heat capacity is $720 \mathrm{~J} / \mathrm{kg}-\mathrm{K}$ [25]. 


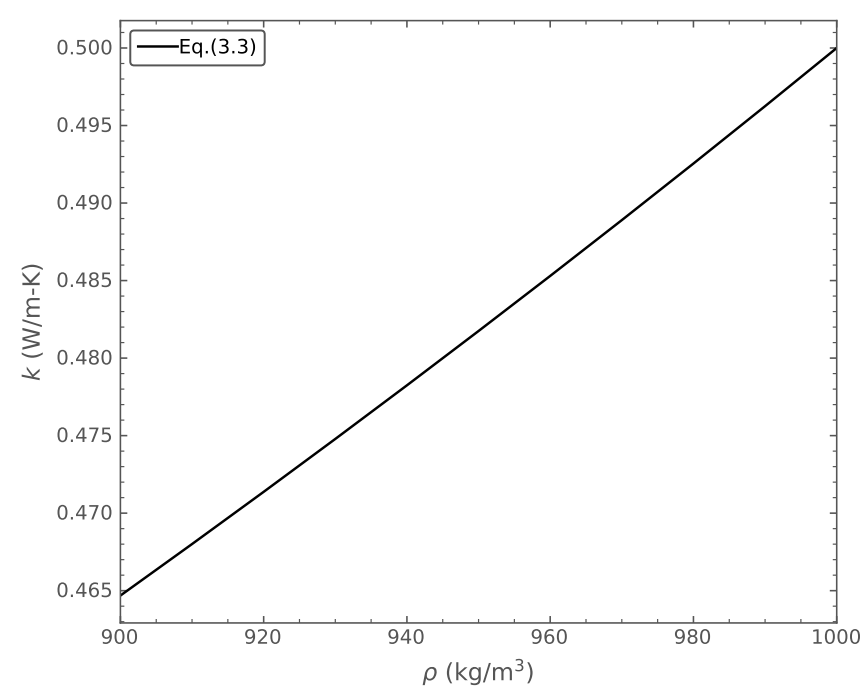

Figure 3.5: The buffer thermal conductivity.

\subsubsection{SiC (MonolithicSiCThermal)}

Three models exist for the thermal conductivity of monolithic SiC: (1) Snead [26], (2) Stone [27], and (3) Miller [23]. The model used in PARFUME as well as for TRISO modeling in BISON is the model by Miller:

$$
k=\frac{17885}{T_{K}}+2.0
$$

where $k(\mathrm{~W} / \mathrm{m}-\mathrm{K})$ is the thermal conductivity and $T_{K}(\mathrm{~K})$ is the temperature.

The correlation for specific heat capacity, $c_{P}(\mathrm{~J} / \mathrm{kg}-\mathrm{K})$, is given by [26]:

$$
c_{P}=925.65+0.3772 T-7.9259 \times 10^{-5} T^{2}-\frac{3.1946 \times 10^{7}}{T^{2}}
$$

where $T(\mathrm{~K})$ is the temperature.

\subsubsection{Graphite matrix (GraphiteMatrixThermal)}

The thermal conductivity of the matrix material is given by $[2,23]$. This correlation of the effective thermal conductivity applies to both the fueled (non-zero packing fraction) and fuelfree regions (zero packing fraction). The thermal conductivity of the fueled zone is based on homogenization of TRISO particles and the matrix to produce an effective thermal conductivity [28]. The correlation is given for fast fluence with a neutron energy threshold of $E>0.18$ $\mathrm{MeV}$. 


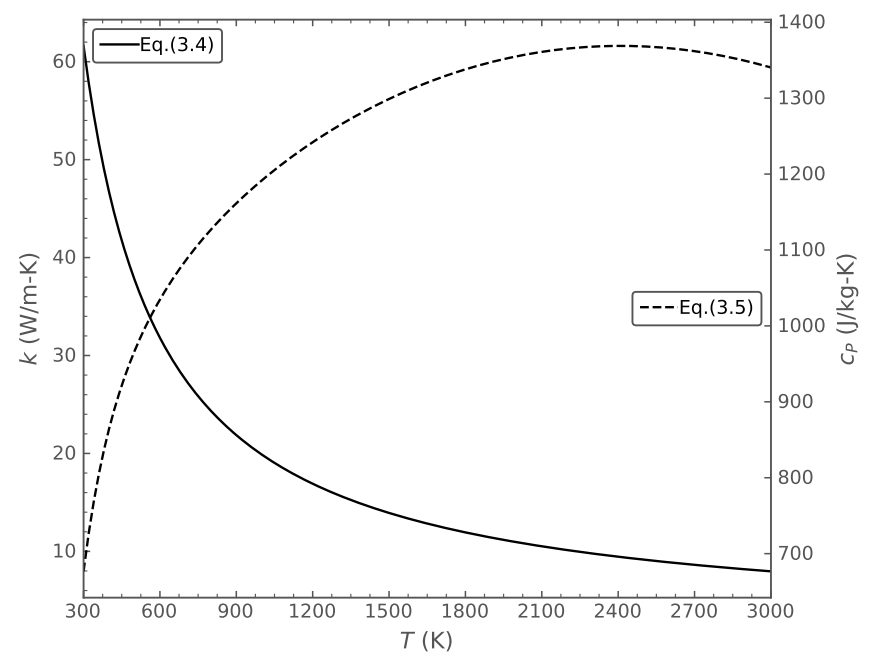

Figure 3.6: Monolithic SiC thermal properties.

The thermal conductivity $k(\mathrm{~W} / \mathrm{m}-\mathrm{K})$ of the matrix is:

$$
k=k_{\text {unirr }} \kappa_{\phi} \kappa_{P F} \kappa_{\rho}
$$

where $k_{\text {unirr }}(\mathrm{W} / \mathrm{m}-\mathrm{K})$ is the temperature-dependent thermal conductivity of the unirradiated matrix material, $\kappa_{\phi}(-)$ is a correction for irradiation damage, $\kappa_{P F}(-)$ is an adjustment for volume loading of TRISO particles (packing fraction), and $\kappa_{\rho}(-)$ is a correction factor for densities other than that of the reference material $\left(1.7 \mathrm{~g} / \mathrm{cm}^{3}\right)$.

The thermal conductivity $k_{\text {unirr }}(\mathrm{W} / \mathrm{m}-\mathrm{K})$ of the unirradiated matrix depends on the nature of the matrix material (A3-3 or A3-27) and temperature of the final heat treatment during fabrication $\left(1800\right.$ or $\left.1950^{\circ} \mathrm{C}\right)[2]$ :

$$
k_{\text {unirr }}=k_{100}(1-\alpha[T-100] \exp (\delta T))
$$

where $k_{100}(\mathrm{~W} / \mathrm{m}-\mathrm{K})$ is the thermal conductivity of non-porous material; the empirical coefficients, $\alpha(-)$ and $\delta(-)$, are tabulated in Table 3.1 ; and $T\left({ }^{\circ} \mathrm{C}\right)$ is the temperature of the matrix. A3-3, heat-treated at $1800^{\circ} \mathrm{C}$, is used by default.

Table 3.1: Coefficients for unirradiated thermal conductivity [2].

\begin{tabular}{ccccc}
\hline Material & $\mathbf{T}\left({ }^{\circ} \mathbf{C}\right)$ & $\mathbf{k}_{\mathbf{1 0 0}}(\mathbf{W} / \mathbf{m}-\mathbf{K})$ & $\alpha$ & $\delta$ \\
\hline A3-3 & 1800 & 50.8 & $1.1810 \times 10^{-3}$ & $-7.8453 \times 10^{-4}$ \\
A3-3 & 1950 & 64.6 & $1.4079 \times 10^{-3}$ & $-9.0739 \times 10^{-4}$ \\
A3-27 & 1800 & 47.4 & $9.7556 \times 10^{-4}$ & $-6.0360 \times 10^{-4}$ \\
A3-27 & 1950 & 62.2 & $1.4621 \times 10^{-3}$ & $-9.6050 \times 10^{-4}$ \\
\hline
\end{tabular}

Neutron fluence decreases the thermal conductivity of the matrix. A correction factor, $\kappa_{\phi}(-)$, 
based on experimental data is applied to take the effect of irradiation damage into account [2], given by:

$$
\kappa_{\phi}=1.0-\left(0.94-0.604 t_{C}\right)\left(1-e^{\left[-\left(2.96-1.955 t_{C}\right) \frac{\phi}{1.52}\right]}\right)-\left(0.043 t_{C}-0.008 t_{C}^{8}\right) \frac{\phi}{1.52}
$$

where $t_{C}=T / 1000, T$ is temperature $\left({ }^{\circ} \mathrm{C}\right)$, and $\phi\left(10^{25} \mathrm{n} / \mathrm{m}^{2}, \mathrm{E}>0.18 \mathrm{MeV}\right)$ is the fast neutron fluence. Note that in the original reference [2], $T$ is mistakenly used instead of $\Gamma$ in the first instance of $\phi$ in Eq. 3.8.

The presence of TRISO particles in the matrix modifies its thermal conductivity. A correction factor, $\kappa_{P F}(-)$, based on the packing fraction, $P F(-)$, is used to take that into account [28]:

$$
\kappa_{P F}=\frac{1+2 \beta P F+\left(2 \beta^{3}-0.1 \beta\right) P F^{2}+0.05 P F^{3} \exp (4.5 \beta)}{1-\beta P F}
$$

with

$$
\beta=\frac{k_{p}-k_{\text {unirr }}}{k_{p}+2 k_{\text {unirr }}}
$$

where $\beta$ depends on the thermal conductivity of the TRISO particles $\left(k_{p}\right)$ and the unirradiated matrix $\left(k_{\text {unirr }}\right)$.

The thermal conductivity of a TRISO particle was estimated based on its geometry and the thermal conductivities of all its constituent layers [28]. A value of $4.13 \mathrm{~W} / \mathrm{m}-\mathrm{K}$ was adopted by [28].

The packing fraction is defined as the ratio of the volume of the TRISO particles to the volume of the embedding matrix:

$$
P F=\frac{N_{p} V_{p}}{V_{f r}},
$$

where $N_{p}(-)$ is the number of TRISO particles embedded in the matrix's fueled region, $V_{p}\left(\mathrm{~cm}^{3}\right)$ is the average volume of a TRISO particle, and $V_{f r}\left(\mathrm{~cm}^{3}\right)$ is the volume of the matrix's fueled region (i.e., the volume containing TRISO particles). If the fuel element (compact or pebble) contains a fuel-free region, the volume of that region is not included in the calculation of the packing fraction. The packing fraction is a fuel specification (i.e., a user input). The packing fraction correction factor differs from the factor presented in [2], which does not apply to packing fractions higher than $10 \%$.

Finally, the correction factor $\kappa_{\rho}(-)$ is applied for densities other than that the reference material $\left(1.7 \mathrm{~g} / \mathrm{cm}^{3}\right)$ used to obtain the experimental data on unirradiated material. It is given by [23]:

$$
\kappa_{\rho}=\frac{\rho}{1700}
$$

where $\rho\left(\mathrm{kg} / \mathrm{m}^{3}\right)$ is the density of the matrix. 


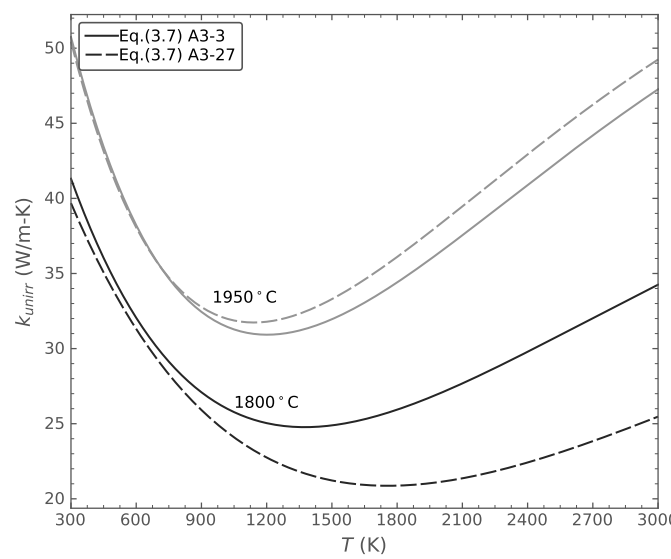

(a)

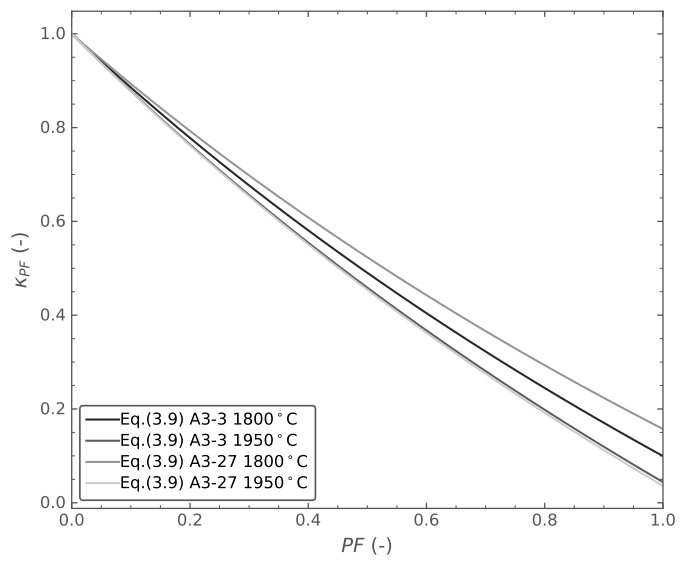

(c)

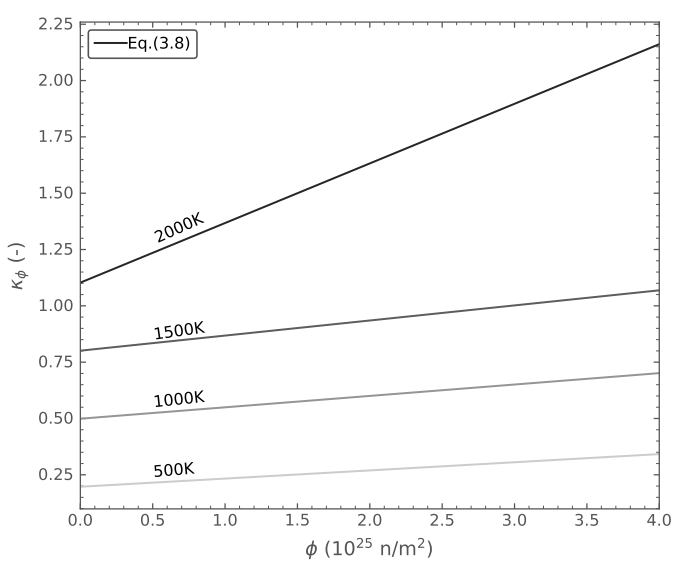

(b)

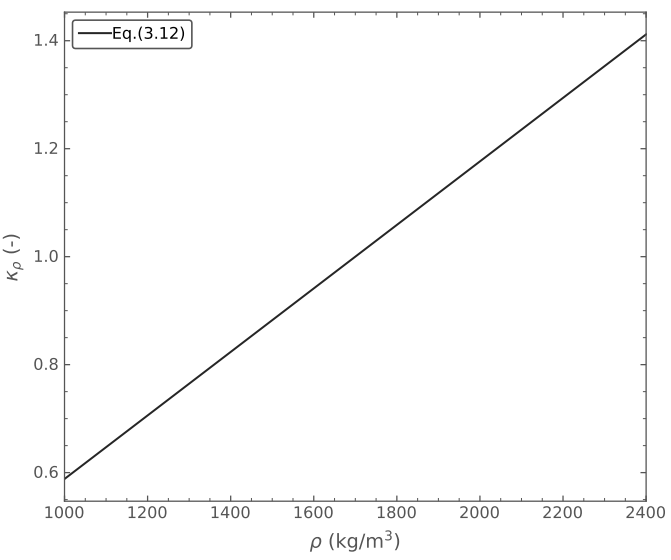

(d)

Figure 3.7: Components of the graphite matrix thermal conductivity: (a) The unirradiated graphite matrix thermal conductivity. (b) The correction factor for the irradiation damage. (c) The correction factor for the particle packing. (d) The correction factor for the density. 
The specific heat capacity of the matrix is given by [29] for carbon brick as:

$$
c_{P}=\frac{1.75 \times 10^{6}}{\rho}\left(0.645+3.14 t_{C}-2.809 t_{C}^{2}+0.959 t_{C}^{3}\right)
$$

where $c_{P}(\mathrm{~J} / \mathrm{kg}-\mathrm{K})$ is the specific heat of the matrix, $\rho\left(\mathrm{kg} / \mathrm{m}^{3}\right)$ is the density of the matrix, $t_{C}=T / 1000$, and $T$ is temperature $\left({ }^{\circ} \mathrm{C}\right)$. If Eq. 3.13 is greater than the maximum allowed value $\left(3.54 \times 10^{3} \mathrm{~J} / \mathrm{kg}-\mathrm{K}\right)$ defined by [2], the maximum value is applied.

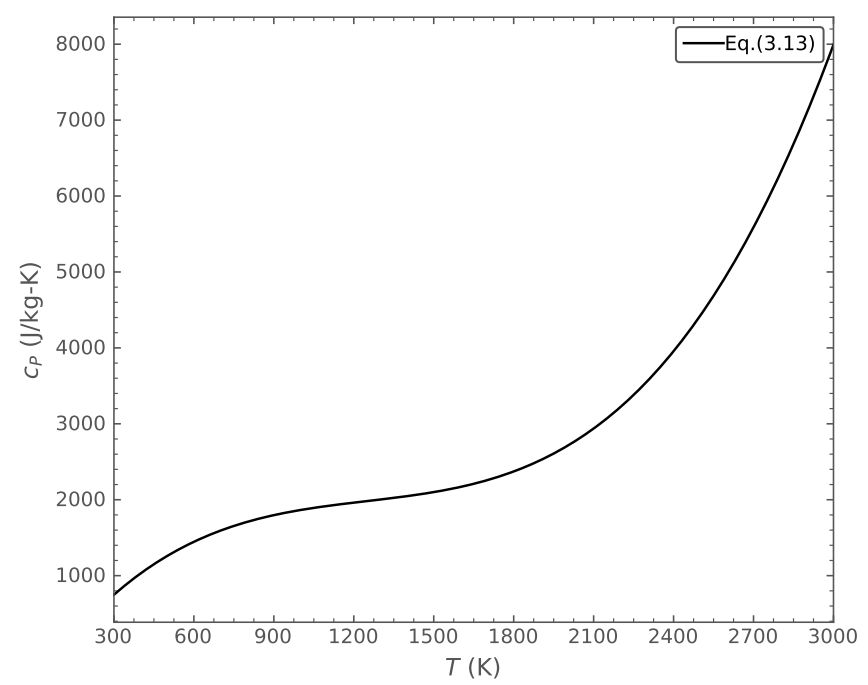

Figure 3.8: The graphite matrix specific heat capacity, computed using an arbitrarily chosen density of $1.7 \mathrm{~g} / \mathrm{cm}^{3}$.

\subsubsection{Mechanical Properties}

\subsubsection{Kernel (UCOElasticityTensor, UCOVolumetricSwellingEigenstrain)}

Elasticity tensor Elastic properties of UCO are given by [30]. These correlations are based on $\mathrm{UO}_{2}$ and used for $\mathrm{UCO}$ due to the lack of experimental data.

$$
\begin{aligned}
E=219\left(1.0-1.1 \times 10^{-4} T_{C}-2.1 \times 10^{-7} T_{C}^{2}+\right. & 3.1 \times 10^{-10} T_{C}^{3} \\
& \left.-1.6 \times 10^{-13} T_{C}^{4}\right)\left(\frac{1.92 \rho-0.92 \rho_{t h}}{\rho_{t h}}\right)
\end{aligned}
$$

where $E(\mathrm{GPa})$ is the elastic modulus of the kernel, $T_{C}\left({ }^{\circ} \mathrm{C}\right)$ is the temperature of the kernel, $\rho\left(\mathrm{g} / \mathrm{cm}^{3}\right)$ is the density of the kernel, and $\rho_{t h}\left(\mathrm{~g} / \mathrm{cm}^{3}\right)$ is the theoretical density of UCO. See Section 3.2.5 for information about the theoretical density of UCO.

Poisson's ratio of the kernel, $v(-)$, is given by: 


$$
v=1.35\left(\frac{1.92 \rho-0.92 \rho_{t h}}{1.66 \rho-0.66 \rho_{t h}}\right)-1
$$

where $\rho\left(\mathrm{g} / \mathrm{cm}^{3}\right)$ is the density of the kernel and $\rho_{t h}\left(\mathrm{~g} / \mathrm{cm}^{3}\right)$ is the theoretical density of UCO.

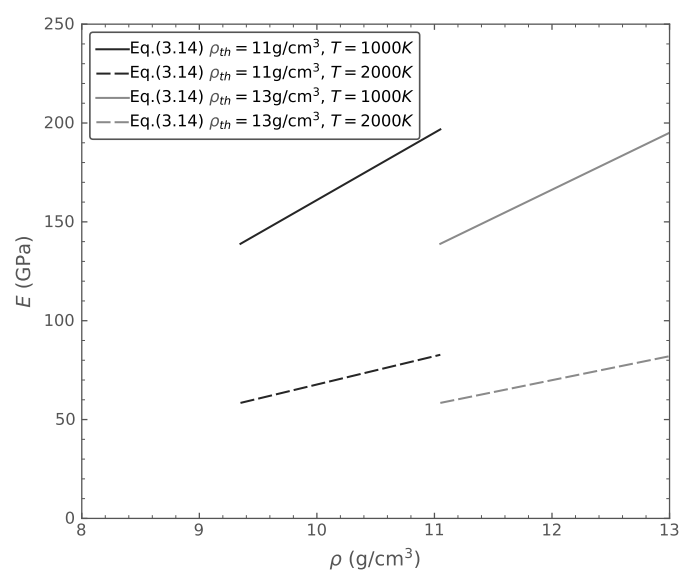

(a) Elastic modulus

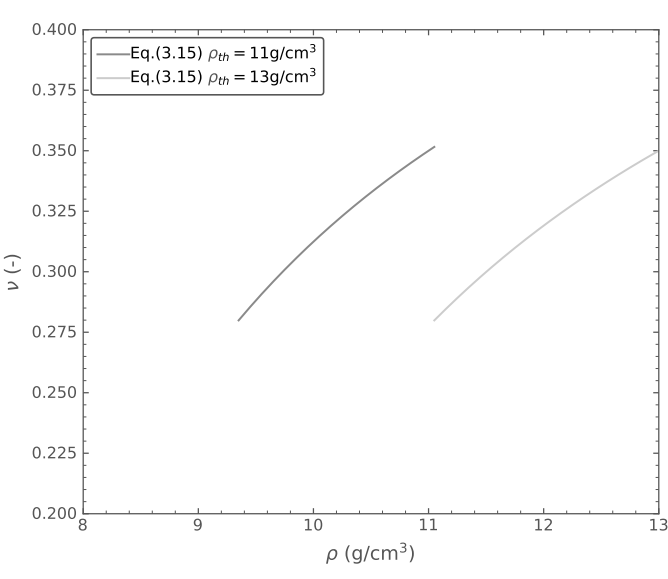

(b) Poisson's ratio

Figure 3.9: UCO kernel elastic properties. Note that temperature and theoretical density values are arbitrarily chosen.

Thermal expansion The thermal expansion coefficient is $10 \times 10^{-6}(1 / \mathrm{K})[30]$.

Volumetric fission-induced swelling The volumetric fission-induced swelling of UCO is given by [23]. The model assumes a constant rate of volume increase due to fission. The functional relationship is derived from experimental data on $\mathrm{UO}_{2}$ and assumed valid for UCO due to the lack of relevant UCO data.

$$
\Delta \varepsilon_{s w}=0.8 \Delta B u
$$

where $\Delta \varepsilon_{s w}(-)$ is the volumetric swelling increment of the kernel and $\Delta B u$ (fissions/atoms-U) is the burnup increment.

\subsubsection{Buffer (BufferElasticityTensor, BufferCEGACreep, BufferThermalExpansionEigenstrain, BufferCEGAIrradiationEigenstrain)}

Elasticity tensor The elastic modulus and Poisson's ratio of the buffer are given by [23, 24].

$$
E=25.5\left(0.384+0.32410^{-3} \rho\right)(1.0+0.23 \phi)\left(1.0+1.510^{-4}\left[T_{C}-20\right]\right)
$$


where $E(\mathrm{GPa})$ is the elastic modulus of the buffer, $\rho\left(\mathrm{kg} / \mathrm{m}^{3}\right)$ is the density of the buffer, $\phi\left(10^{25}\right.$ $\left.\mathrm{n} / \mathrm{m}^{2}, \mathrm{E}>0.18 \mathrm{MeV}\right)$ is the fast neutron fluence, and $T_{C}\left({ }^{\circ} \mathrm{C}\right)$ is the temperature of the buffer.

Poisson's ratio of the buffer is set to a constant value of 0.33 .

Irradiation-induced creep The irradiation-induced creep coefficient for creep of the buffer is given by [23, 24]:

$$
K_{S}=C_{a m p}(1+2.38[1.9-\rho])\left(2.193 \times 10^{-29}-4.85 \times 10^{-32} T_{C}+4.0147 \times 10^{-35} T_{C}^{2}\right)
$$

Here, $K_{S}\left(\mathrm{~m}^{2} / \mathrm{MPa}-\mathrm{n}\right)$ is the irradiation-induced creep coefficient of the buffer, assumed to be independent of fast fluence; $C_{a m p}$ is a creep amplification coefficient used to vary the amplitude of the irradiation-induced creep to account for uncertainty in its value and is set to a default value of 2.0 to better match experimental data from the New Production Reactor program; $\rho\left(\mathrm{g} / \mathrm{cm}^{3}\right)$ is the density of the buffer; and $T_{C}\left({ }^{\circ} \mathrm{C}\right)$ is the temperature of the buffer.

Poisson's ratio for creep of the buffer is set to a constant value of 0.5 [23, 24].

For more information about creep in the buffer, see [19].

Thermal expansion The isotropic coefficient of thermal expansion of the buffer is given by $[23,24]$ :

$$
\alpha=5\left(1+0.11\left[\frac{T_{C}-400}{700}\right]\right)
$$

where $\alpha\left(10^{-6} / \mathrm{K}\right)$ is the coefficient of thermal expansion of the buffer (see Fig. 3.10b) and $T_{C}$ $\left({ }^{\circ} \mathrm{C}\right)$ is the temperature of the buffer. The thermal expansion strain is derived from the isotropic coefficient of thermal expansion using a total formulation based on the difference between the current and stress-free temperatures:

$$
\varepsilon_{t e}=\alpha\left(T_{K}-T_{\text {StressFree }}\right)
$$

where $\varepsilon_{t e}(-)$ is the thermal expansion strain of the buffer, $\alpha\left(10^{-6} / \mathrm{K}\right)$ is the coefficient of thermal expansion of the buffer, $T_{K}(\mathrm{~K})$ is the temperature of the buffer, and $T_{\text {StressFree }}(\mathrm{K})$ is the stressfree temperature.

Irradiation eigenstrain The irradiation eigenstrain of the buffer is given by [23, 24]. First, an isotropic strain $\varepsilon_{i s o}(\%)$ is computed:

$$
\varepsilon_{i s o}=a_{1} \phi+a_{2} \phi^{2}+a_{3} \phi^{3}+a_{4} \phi^{4}
$$

where $\phi\left(\times 10^{25} \mathrm{n} / \mathrm{m}^{2}, \mathrm{E}>0.18 \mathrm{MeV}\right)$ is the fast neutron fluence and $a_{1}$ through $a_{4}$ are temperaturedependent polynomial coefficients, as given in Fig. 3.14. The polynomial coefficients at other temperatures are interpolated or extrapolated from Fig. 3.14. 


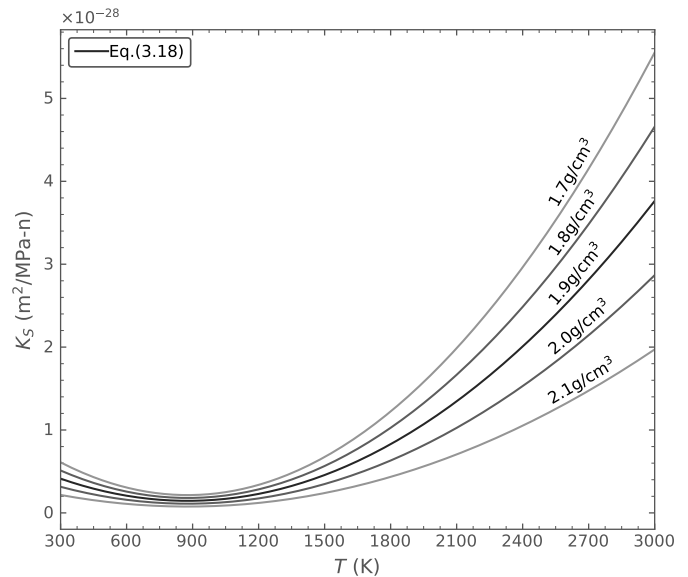

(a) Irradiation-induced creep coefficient.

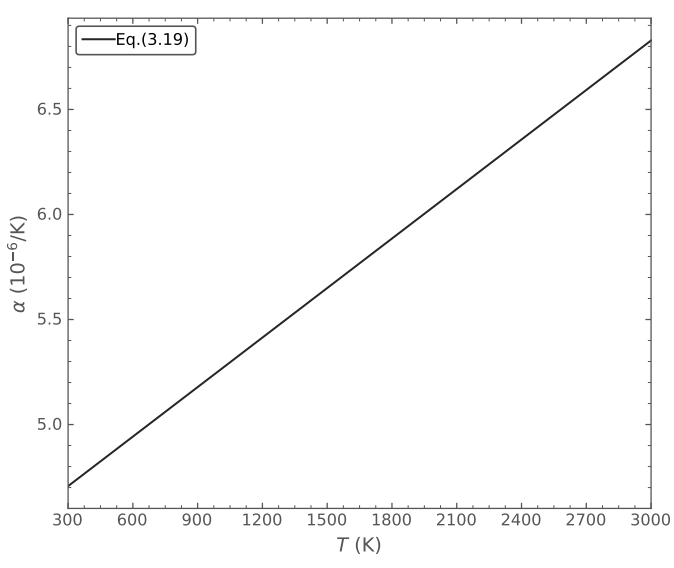

(b) Thermal expansion coefficient.

Figure 3.10: The buffer mechanical properties. Note that temperature and theoretical density values are arbitrarily chosen.

$\varepsilon_{i s o}$ is then adjusted by applying the multiplier $\mu$ :

$$
\mu=\frac{f(\rho)}{f\left(\rho_{0}=1.96\right)}
$$

where $\rho\left(\mathrm{g} / \mathrm{cm}^{3}\right)$ is the density of the buffer.

The isotropic density scaling factor is calculated using the values in Table 3.2, which shows the relationship between the isotropic strain and density at an irradiation temperature of $1100^{\circ} \mathrm{C}$ and a fast fluence of $3.7 \times 10^{25} \mathrm{n} / \mathrm{m}^{2}(\mathrm{E}>0.18 \mathrm{MeV})$.

Table 3.2: Isotropic strain at irradiation temperature of $1100^{\circ} \mathrm{C}$ and fast fluence of $3.7 \times 10^{25}$ $\mathrm{n} / \mathrm{m}^{2}(\mathrm{E}>0.18 \mathrm{MeV})$.

\begin{tabular}{lccccccccc}
\hline$\rho\left(\mathrm{g} / \mathbf{c m}^{3}\right)$ & $\mathbf{1 . 0}$ & $\mathbf{1 . 2}$ & $\mathbf{1 . 4}$ & $\mathbf{1 . 5}$ & $\mathbf{1 . 6}$ & $\mathbf{1 . 8}$ & $\mathbf{1 . 9}$ & $\mathbf{1 . 9 6}$ & $\mathbf{2 . 0}$ \\
\hline$f(\rho)$ & -16.15 & -13.11 & -9.98 & -8.93 & -6.97 & -4.42 & -3.41 & -2.75 & -2.33 \\
\hline
\end{tabular}




\subsubsection{PyC (PyCElasticityTensor, PyCCEGACreep, PyCThermalExpansionEigenstrain, PyCCEGAIrradiationEigenstrain)}

Elasticity tensor The elastic modulus and Poisson's ratio of the PyC layers are given by [23, 24]. The elastic modulus, $E$ (GPa), is computed as:

$$
E=\frac{E_{r}+E_{t}}{2},
$$

with its radial and tangential components, $E_{r}$ and $E_{t}$, as:

$$
\begin{gathered}
E_{r}=25.5\left(0.384+0.324 \times 10^{-3} \rho\right)\left(1.463-0.463 B A F_{0}\right)\left(2.985-0.0662 L_{c}\right) \\
(1+0.23 \phi)[1+0.00015(T-20)] \\
E_{t}=25.5\left(0.384+0.324 \times 10^{-3} \rho\right)\left(0.481+0.519 B A F_{0}\right)\left(2.985-0.0662 L_{c}\right) \\
(1+0.23 \phi)[1+0.00015(T-20)]
\end{gathered}
$$

where $\rho\left(\mathrm{kg} / \mathrm{m}^{3}\right)$ is the density, $B A F_{0}(-)$ is the as-fabricated Bacon anisotropy factor (BAF), $L_{c}$ $(\AA)$ is the crystallite diameter, $\phi\left(\times 10^{25} \mathrm{n} / \mathrm{m}^{2}, \mathrm{E}>0.18 \mathrm{MeV}\right)$ is the fast neutron fluence, and $T$ $\left({ }^{\circ} \mathrm{C}\right)$ is the temperature. The default value of $L_{c}$ is set to be $30 \AA$, such that the factor of that term takes on a value of 1 .

Poisson's ratio is set to a constant value of 0.33 .

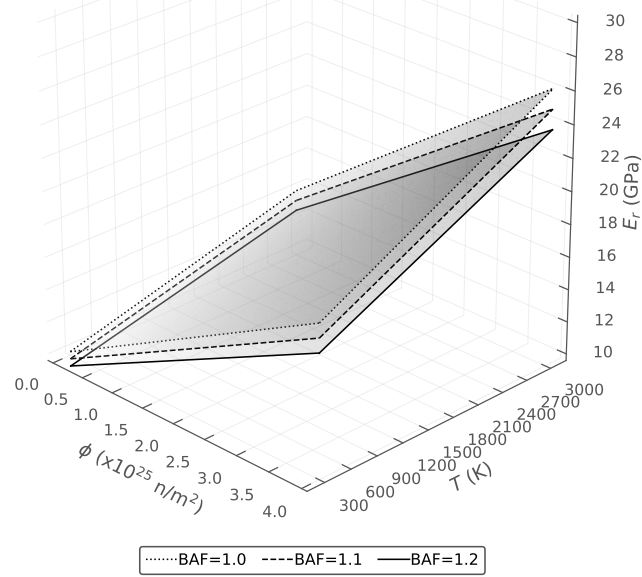

(a) Radial.

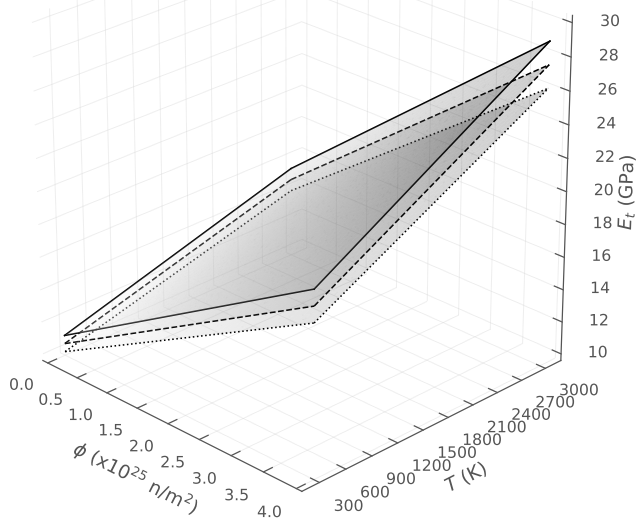

(b) Tangential.

Figure 3.11: PyC elastic modulus. 
Irradiation-induced creep The irradiation creep correlation for PyC is taken from [18, 31]. With $K\left(\mathrm{~m}^{2} / \mathrm{MPa}-\mathrm{n}\right)$ as the creep constant, $\sigma_{i}$ as one component of the principal stress, $\mathrm{v}_{c}$ as Poisson's ratio for creep, and $\phi\left(\times 10^{25} \mathrm{n} / \mathrm{m}^{2}, \mathrm{E}>0.18 \mathrm{MeV}\right)$ as the fast neutron fluence, the creep rate is computed as:

$$
\dot{\varepsilon}_{1}=K\left[\sigma_{1}+v_{c}\left(\sigma_{2}+\sigma_{3}\right)\right] \dot{\phi},
$$

where

$$
K=K_{0}[1+2.38(1.9-\rho)] M_{\text {Irr,Creep }}
$$

The steady-state creep coefficient, $K_{0}$, is given by [23] as:

$$
K_{0}=2.193 \times 10^{-29}-4.85 \times 10^{-32} T+4.0147 \times 10^{-35} T^{2} .
$$

Here, $M_{\text {Irr,Creep }}=2, \rho$ is in $\mathrm{g} / \mathrm{cm}^{3}$, and $T$ is in ${ }^{\circ} \mathrm{C}$. At the expense of inverting a $3 \times 3$ matrix, it is possible to determine the creep strain increment implicitly, allowing arbitrarily large time steps without any unstable creep response.

Poisson's ratio for creep of the PyC layers is set to a constant value of 0.5.

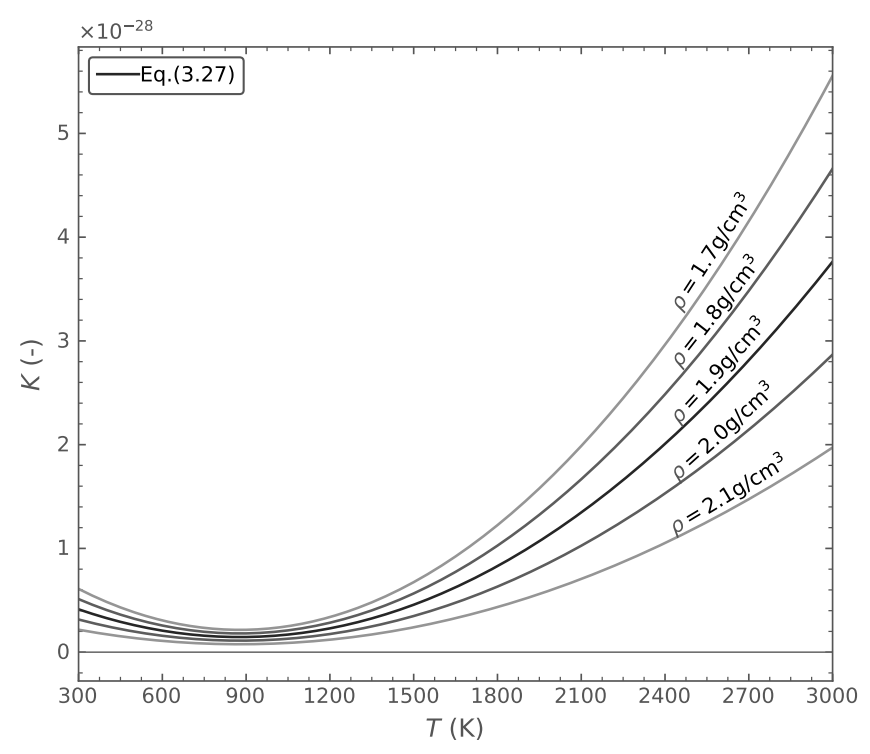

Figure 3.12: PyC creep coefficient.

Thermal expansion The radial and tangential coefficients of thermal expansion of PyC are given by [23]. The thermal expansion strains, $\varepsilon_{i}(-)$, for $i=$ radial,tangential are:

$$
\varepsilon_{i}=\alpha_{i}\left(T-T_{\text {StressFree }}\right),
$$

where $\alpha_{i}$ is the thermal expansion coefficient $(1 / \mathrm{K}), T(\mathrm{~K})$ is the temperature, and $T_{\text {StressFree }}(\mathrm{K})$ is the stress-free temperature. 
Thermal expansion coefficients, $\alpha_{i}\left(10^{-6} / \mathrm{K}\right)$, for $i=$ radial,tangential are:

$$
\begin{gathered}
\alpha_{r}=\left(30-37.5 R_{r}\right)\left(1+0.11\left[\frac{T-673}{700}\right]\right), \\
\alpha_{t}=\left(36\left[R_{t}-1\right]^{2}+1\right)\left(1+0.11\left[\frac{T-673}{700}\right]\right),
\end{gathered}
$$

with

$$
R_{r}=\frac{2}{2+B A F}, \quad R_{t}=\frac{1+B A F}{2+B A F},
$$

where $R_{i}(-)$ is the orientation parameter in the $i$-th direction, $T(\mathrm{~K})$ is the temperature, and $B A F$ is the BAF (see Section 3.2.5).

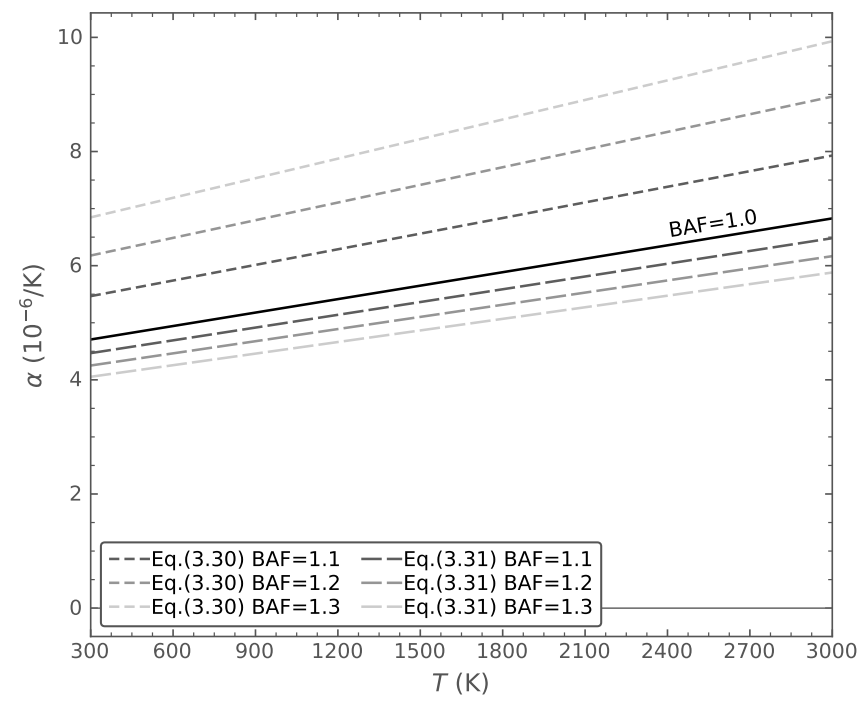

Figure 3.13: PyC thermal expansion coefficients.

Irradiation eigenstrain The irradiation eigenstrain of the PyC layers is given by [23, 24]. The strains, $\varepsilon_{i}(\%)$, for $i=$ radial,tangential,isotropic are given by:

$$
\varepsilon_{i}=a_{1} \phi+a_{2} \phi^{2}+a_{3} \phi^{3}+a_{4} \phi^{4},
$$

where $\phi\left(10^{25} \mathrm{n} / \mathrm{m}^{2}, \mathrm{E}>0.18 \mathrm{MeV}\right)$ is the fast neutron fluence and $a_{1}$ through $a_{4}$ are BAF- and temperature-dependent polynomial coefficients $\left(\rho_{0}=1.96 \mathrm{~g} / \mathrm{cm}^{3}\right)$ at varied temperatures. These are tabulated below.

The polynomial coefficients at other BAFs and temperatures are interpolated or extrapolated from these tables. The isotropic strain $(\mathrm{BAF}=1.0)$ is calculated at the temperature of the PyC 


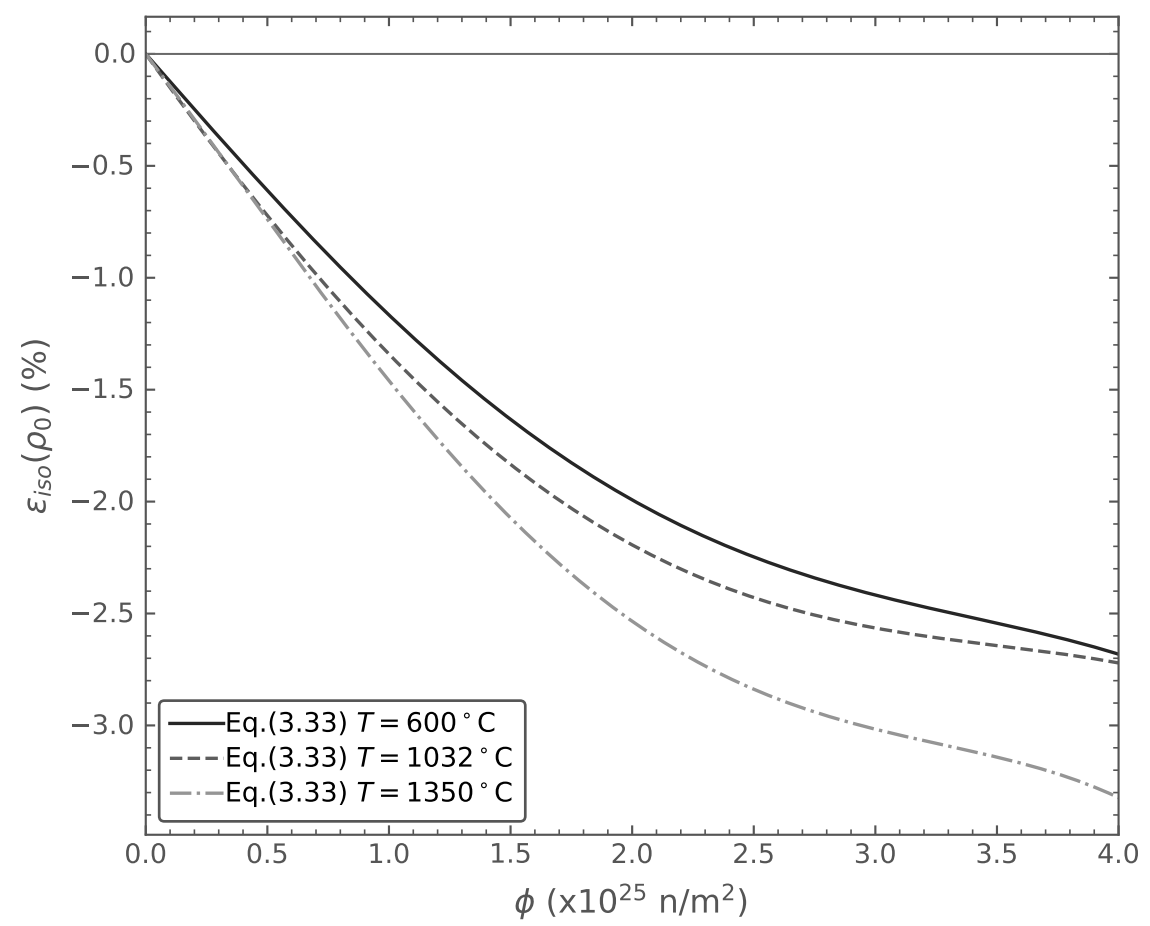

Figure 3.14: Buffer and PyC isotropic irradiation strain at $\rho_{0}=1.96 \mathrm{~g} / \mathrm{cm}^{3}$. Polynomial coefficients for isotropic strain components are tabulated as follows:

\begin{tabular}{cccccc}
\hline $\mathbf{T}\left({ }^{\circ} \mathbf{C}\right)$ & $\mathbf{B A F}$ & $\mathbf{a}_{\mathbf{1}}$ & $\mathbf{a}_{\mathbf{2}}$ & $\mathbf{a}_{3}$ & $\mathbf{a}_{\mathbf{4}}$ \\
\hline 600 & 1.0000 & -1.24080 & 0.00175 & 0.08533 & -0.01253 \\
1032 & 1.0000 & -1.52390 & 0.13048 & 0.06299 & -0.01072 \\
1350 & 1.0000 & -1.42840 & -0.19563 & 0.18991 & -0.02591 \\
\hline
\end{tabular}




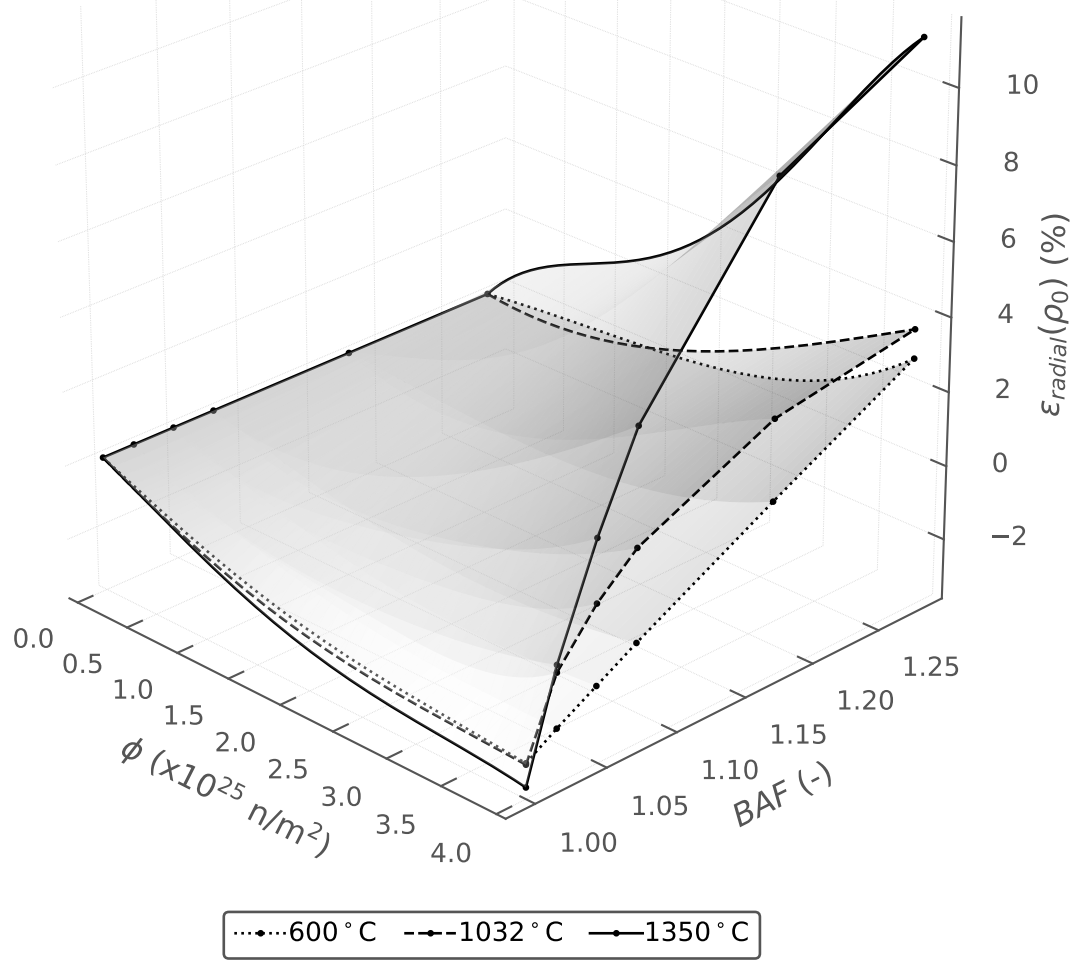

Figure 3.15: PyC radial irradiation strain at $\rho_{0}=1.96 \mathrm{~g} / \mathrm{cm}^{3}$. Polynomial coefficients for radial strain components are tabulated as follows:

\begin{tabular}{cccccccc}
\hline & $\mathbf{B A F}=$ & $\mathbf{1 . 0 0 0 0}$ & $\mathbf{1 . 0 2 1 2}$ & $\mathbf{1 . 0 4 8 8}$ & $\mathbf{1 . 0 7 6 9}$ & $\mathbf{1 . 1 7 4 6}$ & $\mathbf{1 . 2 7 8 7}$ \\
\hline $600^{\circ} \mathrm{C}$ & $a_{1}$ & -1.24080 & -1.10640 & -0.94333 & -0.78045 & -0.15714 & 0.40265 \\
& $a_{2}$ & 0.00175 & -0.03128 & -0.03589 & -0.02975 & -0.14889 & -0.16501 \\
& $a_{3}$ & 0.08533 & 0.09184 & 0.08184 & 0.06655 & 0.07546 & 0.03676 \\
& $a_{4}$ & -0.01253 & -0.01220 & -0.00958 & -0.00626 & -0.00293 & 0.00706 \\
\hline $1032^{\circ} \mathrm{C}$ & $a_{1}$ & -1.52390 & -2.07520 & -2.00470 & -1.81690 & -1.18540 & -0.45900 \\
& $a_{2}$ & 0.13048 & 1.37845 & 1.30380 & 1.10850 & 0.64995 & 0.51172 \\
& $a_{3}$ & 0.06299 & -0.48993 & -0.37280 & -0.23868 & 0.01380 & -0.03245 \\
& $a_{4}$ & -0.01072 & 0.06602 & 0.04538 & 0.02484 & -0.01284 & -0.00142 \\
\hline $1350^{\circ} \mathrm{C}$ & $a_{1}$ & -1.42840 & -1.54330 & -1.49640 & -0.89522 & 1.20930 & 3.71620 \\
& $a_{2}$ & -0.19563 & 0.59804 & 1.16621 & 0.80331 & -0.53861 & -2.70420 \\
& $a_{3}$ & 0.18991 & -0.09997 & -0.30106 & -0.09009 & 0.43114 & 1.17990 \\
& $a_{4}$ & -0.02591 & 0.00978 & 0.03475 & 0.00467 & -0.05590 & -0.13910 \\
\hline
\end{tabular}




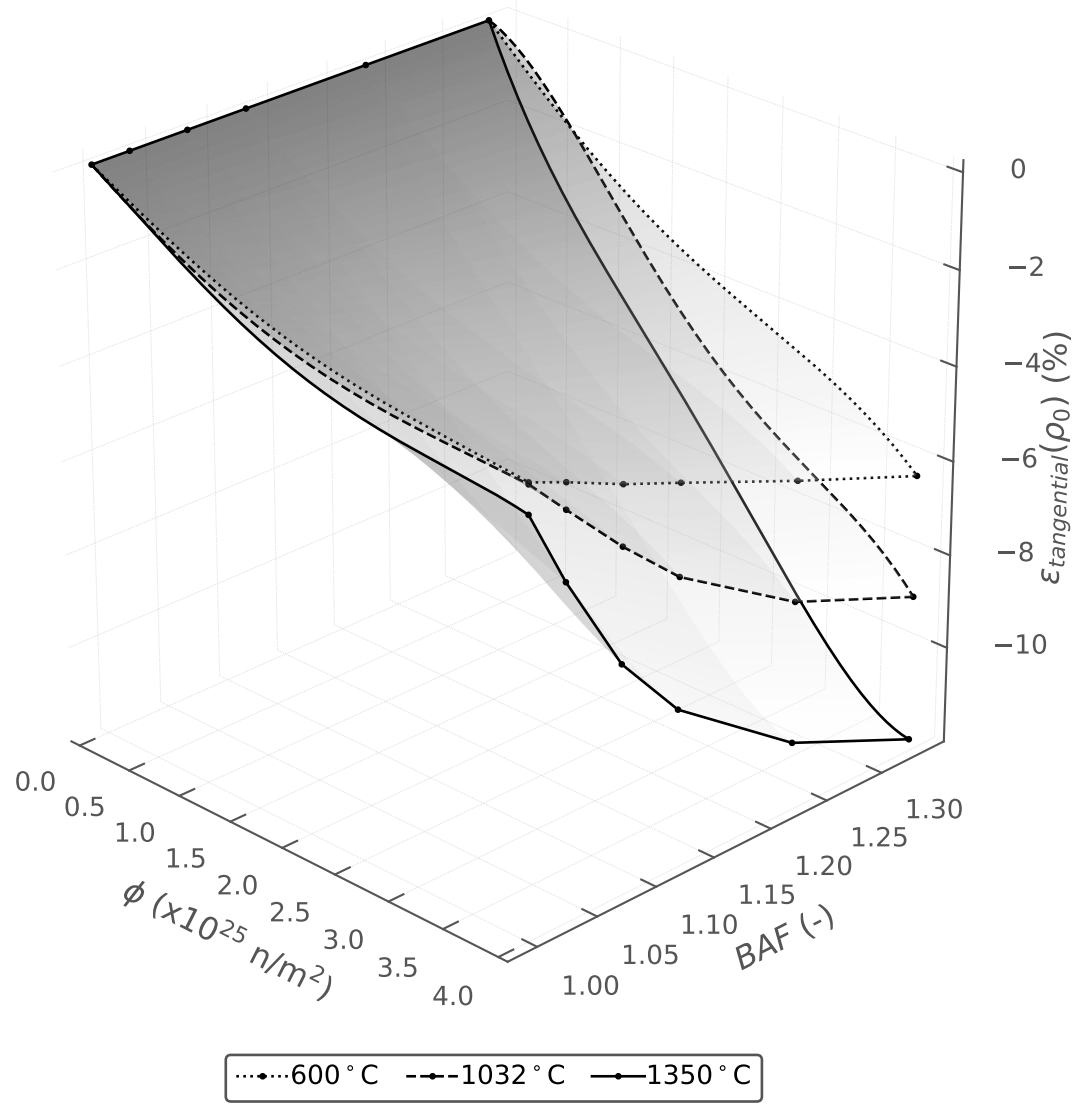

Figure 3.16: PyC tangential irradiation strain at $\rho_{0}=1.96 \mathrm{~g} / \mathrm{cm}^{3}$. Polynomial coefficients for tangential strain components are tabulated as follows:

\begin{tabular}{cccccccc}
\hline & $\mathbf{B A F}=$ & $\mathbf{1 . 0 0 0 0}$ & $\mathbf{1 . 0 3 0 3}$ & $\mathbf{1 . 0 7 6 9}$ & $\mathbf{1 . 1 2 5 0}$ & $\mathbf{1 . 2 2 5 8}$ & $\mathbf{1 . 3 3 3 3}$ \\
\hline \multirow{2}{6}{$600^{\circ} \mathrm{C}$} & $a_{1}$ & -1.24080 & -1.38550 & -1.46790 & -1.64660 & -1.84990 & -2.19190 \\
& $a_{2}$ & 0.00175 & 0.05307 & -0.02836 & 0.03928 & -0.09358 & 0.02675 \\
& $a_{3}$ & 0.08533 & 0.07620 & 0.12139 & 0.10067 & 0.18119 & 0.15352 \\
& $a_{4}$ & -0.01253 & -0.01245 & -0.01948 & -0.01764 & -0.03036 & -0.02972 \\
\hline $1032^{\circ} \mathrm{C}$ & $a_{1}$ & -1.52390 & -1.57590 & -1.32200 & -1.18700 & -0.96963 & -0.81239 \\
& $a_{2}$ & 0.13048 & 0.09019 & -0.51928 & -0.90635 & -1.59110 & -2.20760 \\
& $a_{3}$ & 0.06299 & 0.05306 & 0.27603 & 0.41046 & 0.64689 & 0.88496 \\
& $a_{4}$ & -0.01072 & -0.00815 & -0.03465 & -0.05067 & -0.07682 & -0.10457 \\
\hline \multirow{2}{*}{$1350^{\circ} \mathrm{C}$} & $a_{1}$ & -1.42840 & -2.24680 & -2.82930 & -3.25550 & -4.44780 & -5.67140 \\
& $a_{2}$ & -0.19563 & 0.48243 & 0.76088 & 0.90423 & 1.60320 & 2.41920 \\
& $a_{3}$ & 0.18991 & -0.07687 & -0.22314 & -0.33175 & -0.58683 & -0.86155 \\
& $a_{4}$ & -0.02591 & 0.00464 & 0.02431 & 0.04329 & 0.07458 & 0.10668 \\
\hline
\end{tabular}


Table 3.3: Isotropic strain and difference between radial and tangential strain at an irradiation temperature of $1100^{\circ} \mathrm{C}$ and fast fluence of $3.7 \times 10^{25} \mathrm{n} / \mathrm{m}^{2}(\mathrm{E}>0.18 \mathrm{MeV})$.

\begin{tabular}{lccccccccc}
\hline$\rho\left(\mathbf{g} / \mathbf{c m}^{\mathbf{3}}\right)$ & $\mathbf{1 . 0 0}$ & $\mathbf{1 . 2 0}$ & $\mathbf{1 . 4 0}$ & $\mathbf{1 . 5 0}$ & $\mathbf{1 . 6 0}$ & $\mathbf{1 . 8 0}$ & $\mathbf{1 . 9 0}$ & $\mathbf{1 . 9 6}$ & $\mathbf{2 . 0 0}$ \\
\hline$\varepsilon_{i s o}(\%)$ & -16.15 & -13.11 & -9.98 & -8.93 & -6.97 & -4.42 & -3.41 & -2.75 & -2.33 \\
$\varepsilon_{r}-\varepsilon_{t}(\%)$ & 0.000 & 0.500 & 1.100 & 1.650 & 2.450 & 6.305 & 7.900 & 9.600 & 11.100 \\
\hline
\end{tabular}

and adjusted for density by applying an isotropic scaling factor, $\mu$. The isotropic scaling factorthe ratio of the isotropic strain at a given density to the isotropic strain at $\rho_{0}$-is computed as:

$$
\mu=\frac{\varepsilon_{i s o}(\rho)}{\varepsilon_{i s o}\left(\rho_{0}\right)} .
$$

The isotropic scaling factor is calculated using the values in Table 3.3, which shows the relationship between the isotropic strain and density at a specified irradiation temperature and fast fluence. Adjustments for density at other BAF values are made by applying an anisotropic scaling factor, $v$. This anisotropic scaling factor - the ratio of the difference between the radial and tangential strain components at a given density to the difference between the radial and tangential strain components at $\rho_{0}$-is computed as:

$$
v=\frac{\varepsilon_{r}(\rho)-\varepsilon_{t}(\rho)}{\varepsilon_{r}\left(\rho_{0}\right)-\varepsilon_{t}\left(\rho_{0}\right)} .
$$

The anisotropic scaling factor is calculated using the values in Table 3.3, which shows the relationship between the difference in the radial and tangential strain components and density at an irradiation temperature and a fast fluence. Thus, the adjusted radial and tangential strains become:

$$
\begin{aligned}
& \varepsilon_{r}(\rho)=\mu \varepsilon_{i s o}\left(\rho_{0}\right)+v\left[\varepsilon_{r}\left(\rho_{0}\right)-\varepsilon_{i s o}\left(\rho_{0}\right)\right] \\
& \varepsilon_{t}(\rho)=\mu \varepsilon_{i s o}\left(\rho_{0}\right)+v\left[\varepsilon_{t}\left(\rho_{0}\right)-\varepsilon_{i s o}\left(\rho_{0}\right)\right]
\end{aligned}
$$

\subsubsection{SiC (MonolithicSiCElasticityTensor)}

The elastic modulus and Poisson's ratio for $\mathrm{SiC}$ is given by [23].

The elastic modulus (E) is temperature-dependent and given according to Table 3.4. For values between the tabulated values, linear interpolation is used. For temperatures below $25.0^{\circ} \mathrm{C}$ Young's modulus is taken as $428.0 \mathrm{GPa}$. For temperatures above $1600^{\circ} \mathrm{C}$, a value of $198.0 \mathrm{GPa}$ is used.

Poisson's ratio is set to a constant value of 0.13 .

The thermal expansion coefficient is $4.9 \times 10^{-6}(1 / \mathrm{K})[23,24]$. 
Table 3.4: Elastic modulus of the $\mathrm{SiC}$ as a function of temperature.

\begin{tabular}{lcccc}
\hline T $\left({ }^{\circ} \mathbf{C}\right)$ & 25.0 & 940.0 & 1215.0 & 1600.0 \\
$\mathbf{E}(\mathbf{G P a})$ & 428.0 & 375.0 & 340.0 & 198.0 \\
\hline
\end{tabular}

\subsubsection{Graphite matrix}

The elastic modulus of the matrix is set to $10 \mathrm{GPa}$, an average value of the radial and tangential elastic moduli for A3-3 and A3-27 matrix materials [32].

Poisson's ratio of the matrix is 0.25 [33], a typical value for nuclear-grade graphite.

\subsubsection{Fission Product Diffusion}

Fission product diffusion follows Eq. 1.3. The mass flux $\mathbf{J}$ is:

$$
\mathbf{J}=-D \nabla C
$$

where the diffusion coefficient $D\left(\mathrm{~m}^{2} / \mathrm{s}\right)$ is defined in Arrhenius form as:

$$
D=D_{0} \exp \left(\frac{-Q}{R T}\right)
$$

where $R$ is the universal gas constant. Values of $D_{0}$ and $Q$ for silver, cesium, and strontium are given in Table $3.5[34,35]$.

Table 3.5: Mass diffusion coefficients.

\begin{tabular}{clccc}
\hline & & Kernel $\left(\mathbf{U O}_{2}\right)$ & PyC & SiC \\
\hline \multirow{2}{*}{ Ag } & $D_{0}\left(\mathrm{~m}^{2} / \mathrm{s}\right)$ & $6.7 \times 10^{-9}$ & $5.3 \times 10^{-9}$ & $3.6 \times 10^{-9}$ \\
& $Q_{0}(\mathrm{~kJ} / \mathrm{mol})$ & 165 & 154 & 215 \\
\hline \multirow{2}{*}{$\mathrm{Cs}$} & $D_{0}\left(\mathrm{~m}^{2} / \mathrm{s}\right)$ & $5.6 \times 10^{-8}$ & $6.3 \times 10^{-8}$ & $5.5 \times 10^{-14}$ \\
& $Q_{0}(\mathrm{~kJ} / \mathrm{mol})$ & 209 & 222 & 125 \\
\hline \multirow{2}{*}{$\mathrm{Sr}$} & $D_{0}\left(\mathrm{~m}^{2} / \mathrm{s}\right)$ & $2.2 \times 10^{-3}$ & $2.3 \times 10^{-6}$ & $1.2 \times 10^{-9}$ \\
& $Q_{0}(\mathrm{~kJ} / \mathrm{mol})$ & 488 & 197 & 205 \\
\hline
\end{tabular}

\subsubsection{Fission Gas}

Fission gas release (FGR) represents the amount of fission gas released by the UCO kernel into the void volume of the TRISO particle (kernel and buffer porosities and buffer-IPyC gap). The 
relationships are partly derived from experimental data on $\mathrm{UO}_{2}$ and assumed valid for $\mathrm{UCO}$ due to the lack of relevant UCO data.

The FGR (mol), is calculated from both direct recoil release and diffusive release as:

$$
F G R=\left(R F_{\text {recoil }}+\left[1.0-R F_{\text {recoil }}\right] R F_{\text {Booth }}\right) F G P
$$

where $R F_{\text {recoil }}(-)$ and $R F_{\text {Booth }}(-)$ are the release fractions of fission gas by direct recoil and diffusion, respectively, and $F G P(\mathrm{~mol})$ is the amount of fission gas produced in the kernel.

The model assumes that $100 \%$ of the fission gas is released to the void volume.

\subsubsection{Direct recoil}

Direct kinetic release of fission gases from the kernel to the buffer is accounted for by geometric considerations and fission fragment ranges derived from compiled experimental data [3].

The fission gas mixture is essentially composed of krypton and xenon, with relative fractions of 18.5 and $81.5 \%$, respectively [23]. Hence, the recoil fraction is given by:

$$
R F_{\text {recoil }}=0.185 R F_{\text {recoil }, K r}+0.815 R F_{\text {recoil }, X e}
$$

where $R F_{\text {recoil }, K r}(-)$ and $R F_{\text {recoil }, X e}(-)$ are the respective release fractions of krypton and xenon due to recoil. They depend on the ranges of krypton and xenon in UCO (i.e., $r_{i}$ for $i=\mathrm{Kr}, \mathrm{Xe}$ ) and on the radius of the kernel, $r_{k}(\mathrm{~m})$ :

$$
R F_{\text {recoil }, i}=0.25\left(\frac{r_{k}^{3}-\left[r_{k}-r_{i}\right]^{3}}{r_{k}^{3}}\right) .
$$

The ranges of krypton and xenon in UCO (i.e., $r_{i}$ for $i=\mathrm{Kr}, \mathrm{Xe}$ ) are obtained from their individual ranges in uranium, carbon, and oxygen:

$$
r_{i}=10.0\left(\frac{r_{i, U}+O U r_{i, O}+C U r_{i, C}}{\rho_{k}[1.0+O U+C U]}\right)
$$

where $O U(-)$ and $C U(-)$ are the initial oxygen-to-uranium and carbon-to-uranium ratios and $\rho_{k}$ $\left(\mathrm{g} / \mathrm{cm}^{3}\right)$ is the density of the kernel. Individual ranges are given in Table 3.6.

Table 3.6: Ranges of $\mathrm{Kr}$ and $\mathrm{Xe}$ in $\mathrm{U}, \mathrm{C}$, and $\mathrm{O}$ [3].

\begin{tabular}{lcccccc}
\hline $\begin{array}{l}\text { Fission Product } \\
\text { Medium }\end{array}$ & $\begin{array}{c}\text { krypton } \\
\text { uranium }\end{array}$ & $\begin{array}{c}\text { krypton } \\
\text { carbon }\end{array}$ & $\begin{array}{c}\text { krypton } \\
\text { oxygen }\end{array}$ & $\begin{array}{c}\text { xenon } \\
\text { uranium }\end{array}$ & $\begin{array}{c}\text { xenon } \\
\text { carbon }\end{array}$ & $\begin{array}{c}\text { xenon } \\
\text { oxygen }\end{array}$ \\
\hline Range $\left(\mathbf{m g} / \mathbf{c m}^{\mathbf{2}}\right)$ & 11.7 & 3.3 & 3.1 & 8.3 & 2.3 & 2.2 \\
\hline
\end{tabular}




\subsubsection{Booth model}

Diffusive release through kernel grains to the grain boundaries and subsequent transport through the interconnected porosity is estimated using the Booth equivalent sphere diffusion model [36].

The Booth release fraction is given by:

$$
R F_{\text {Booth }}=1.0-\frac{6.0 r_{\text {grain }}^{2}}{D t} \sum_{n=1}^{\infty} \frac{1.0-\exp \left(\frac{-n^{2} \pi^{2} D t}{r_{\text {grain }}^{2}}\right)}{n^{4} \pi^{4}}
$$

where $r_{\text {grain }}(\mathrm{m})$ is the radius of the diffusing sphere (i.e., the average grain radius), $D\left(\mathrm{~m}^{2} / \mathrm{s}\right)$ is the diffusivity of the fission gas in the grain, and $t(\mathrm{~s})$ is the diffusion time.

The UCO model utilizes an effective diffusion coefficient, $D_{\text {eff }}\left(\mathrm{m}^{2} / \mathrm{s}\right)$, formulated for $\mathrm{UO}_{2}$ fuel by [37] as:

$$
D_{\text {eff }}=\sum_{i=1}^{3} D_{i}=D_{1}+D_{2}+D_{3}
$$

where $D_{1}, D_{2}$, and $D_{3}$ are the coefficients of the mechanisms controlling diffusion at different temperature ranges.

- At the highest temperatures, diffusion proceeds through the cation lattice by means of thermally activated vacancies:

$$
D_{1}=7.6 \times 10^{-10} \exp \left(-\frac{35225}{T_{K}}\right)
$$

where $T_{K}(\mathrm{~K})$ is the temperature of the kernel.

- At intermediate temperatures, diffusion is driven by means of vacancies produced by the irradiation process:

$$
D_{2}=s^{2} j_{v}\left(\frac{K^{\prime}}{Z j_{v}}\right)^{0.5}
$$

where $s(\mathrm{~m})$ is the atomic jump distance, $j_{v}\left(s^{-1}\right)$ is the cation vacancy jump rate, $K^{\prime}\left(s^{-1}\right)$ is the rate of defect production per atom, and $Z(-)$ is the number of sites around a point defect from which recombination is inevitable. They are given by:

$$
\begin{gathered}
s=3.0 \times 10^{-10} \\
j_{v}=10^{13} \exp \left(-\frac{27778}{T_{K}}\right)
\end{gathered}
$$




$$
K^{\prime}=\frac{K B_{u}}{t}
$$

$$
Z=2
$$

where $\mathrm{K}\left(10^{4}\right.$ defects/fission) is the damage rate and $B_{u}$ (fissions per initial metal atom [FIMA]) is the burnup.

- Finally, at lower temperatures, a term proportional to the fission rate density, $f^{\prime \prime \prime}$ (fission $/ \mathrm{m}^{3}$ s), is used to cause agreement with experimental results reported by [37]:

$$
D_{3}=2.0 \times 10^{-40} f^{\prime \prime \prime}
$$

\subsubsection{Fission gas production}

The amount of fission gas produced by the kernel, $F G P(\mathrm{~mol})$, during a time interval $\Delta t(\mathrm{~s})$, is calculated as:

$$
F G P=\frac{\Gamma_{F G} f^{\prime \prime \prime} V_{k} \Delta t}{N_{\text {avo }}}
$$

where $\Gamma_{F G}(-)$ is the combined fractional fission yield of krypton and xenon atoms per fission, $V_{k}\left(\mathrm{~m}^{3}\right)$ is the volume of the kernel, and $N_{\text {avo }}\left(6.022 \times 10^{23} \mathrm{at} / \mathrm{mol}\right)$ is Avogadro's number.

The combined fission yield of $\mathrm{Kr}$ and $\mathrm{Xe}$ is equal to [23]:

$$
\Gamma_{F G}=0.297
$$

See Section 3.2.5 for details about fission yields.

\subsubsection{Miscellaneous}

A handful of miscellaneous models for evaluating TRISO performance include the determination of the molar mass, the calculation of theoretical density, the determination of the fission yields, the BAF calculations, and the Weibull strength parameters.

\subsubsection{Molar mass}

The molar mass of the kernel, $M(\mathrm{~kg} / \mathrm{mol})$, is computed for $i=\mathrm{UO}_{2}, \mathrm{UC}_{2}$, and $\mathrm{UC}$ by:

$$
M=\sum_{i}^{N} a f_{i} \times a w_{i}
$$


with

$$
a f= \begin{cases}0.5 O U & \text { for } \mathrm{UO}_{2} \\ 0.5 O U+C U-1.0 & \text { for } \mathrm{UC}_{2} \\ 2.0-(O U+C U) & \text { for } \mathrm{UC}\end{cases}
$$

and

$$
a w= \begin{cases}0.23504 \varepsilon+0.23805(1.0-\varepsilon)+0.03200 & \text { for } \mathrm{UO}_{2} \\ 0.23504 \varepsilon+0.23805(1.0-\varepsilon)+0.02402 & \text { for } \mathrm{UC}_{2} \\ 0.23504 \varepsilon+0.23805(1.0-\varepsilon)+0.01201 & \text { for } \mathrm{UC}^{2}\end{cases}
$$

where $\varepsilon$ (wt.\%) is the initial U-235 enrichment, $O U(-)$ and $C U(-)$ are the initial oxygen-touranium and carbon-to-uranium ratios of $\mathrm{UCO}, a f(-)$ is the atomic fraction, and $a w(\mathrm{~kg} / \mathrm{mol})$ is the atomic weight.

$\mathrm{UCO}$ is assumed to be formed of $\mathrm{UO}_{2}, \mathrm{UC}_{2}$, and $\mathrm{UC}$. However, $\mathrm{UC}_{2}$ and $\mathrm{UC}$ are known to mix during heat treatment and form a $\mathrm{UC}_{x}$ phase, where $\mathrm{x}<2$.

Atomic weights of the UCO constituents are tabulated in Table 3.7.

Table 3.7: The theoretical densities of the UCO mixture components.

\begin{tabular}{ccccc}
\hline & uranium-235 & uranium-238 & oxygen & carbon \\
\hline$a w(\mathrm{~g} / \mathrm{mol})$ & 235.04 & 238.05 & 16.00 & 12.01 \\
\hline
\end{tabular}

\subsubsection{Theoretical density}

The theoretical density of the kernel, $\rho_{t h}\left(\mathrm{~kg} / \mathrm{m}^{3}\right)$, is calculated as:

$$
\begin{gathered}
\rho_{t h}=\frac{1}{\frac{w f_{U O_{2}}}{\rho_{t h}^{U O_{2}}}+\frac{w f_{U C}}{\rho_{t h}^{U C}}+\frac{w f_{U C_{2}}}{\rho_{t h}^{U C_{2}}}} \\
w f=\frac{a f \cdot a w}{M}
\end{gathered}
$$

where $w f(-)$ is the weight fraction. The quantities $a f$ and $a w$ come from the calculations described in the kernel molar mass section.

The theoretical densities of the UCO mixture components are tabulated in Table 3.8.

\subsubsection{Fission yields}

Fission yields, $\Gamma$ (atoms/fission), for silver, cesium, strontium, and both krypton and xenon combined are given below. 
Table 3.8: The theoretical densities of the UCO mixture components.

\begin{tabular}{cccc}
\hline & $\mathrm{UO}_{2}$ & $\mathrm{UC}$ & $\mathrm{UC}_{2}$ \\
\hline$\rho_{t h}\left(\mathrm{~g} / \mathrm{cm}^{3}\right)$ & 10.96 & 13.63 & 11.28 \\
\hline
\end{tabular}

- For silver:

$$
\Gamma_{A g}= \begin{cases}1.31625 \times 10^{-3} b^{0.55734} & \text { for } \varepsilon<17.5 \\ 8.24492 \times 10^{-4} b^{0.53853} & \text { for } \varepsilon \geq 17.5\end{cases}
$$

with $b=\max (1.0, B u)$.

- For cesium:

$$
\Gamma_{C s}= \begin{cases}0.14 & \text { for } \varepsilon<17.5 \\ 0.16 & \text { for } \varepsilon \geq 17.5\end{cases}
$$

- For strontium:

$$
\Gamma_{S r}= \begin{cases}0.11754 b^{-0.21762} & \text { for } \varepsilon<17.5 \\ 0.11819 b^{-0.15778} & \text { for } \varepsilon \geq 17.5\end{cases}
$$

with $b=\max (0.6, B u)$.

- For krypton and xenon:

$$
\Gamma_{K r+X e}=0.297
$$

where $\mathrm{Bu}(\% \mathrm{FIMA})$ is the burnup and $\varepsilon(\%)$ is the ${ }^{235} \mathrm{U}$ enrichment [23].

\subsubsection{Bacon anisotropy factor}

The BAF is defined based on an $\mathrm{x}$-ray measurement technique. BAF is a direct measure of the crystallographic anisotropy of pyrocarbon coatings deposited on spherical fuel particles. BAF

\begin{tabular}{|c|c|c|c|c|c|c|c|c|}
\hline$\phi\left(\mathbf{x} 10^{25} \mathbf{n} / \mathbf{m}^{2}\right)$ & 0.0 & 1.0 & 2.0 & 3.0 & 3.5 & 4.0 & 4.5 & $\geq \mathbf{5 . 0}$ \\
\hline $\mathbf{B A F} \mathbf{B A F}_{0}$ & 0000 & 1.0019 & 1.0114 & 1.0219 & 1.0286 & 1.0324 & 1.0362 & 1.0381 \\
\hline
\end{tabular}
increases with fast fluence, and the ratio of irradiated to unirradiated BAF values is tabulated in Table 3.9 as a function of fast fluence.

Table 3.9: BAF vs. fast neutron fluence $(\mathrm{E}>0.18 \mathrm{MeV})$. 


\subsubsection{Weibull strength parameters}

The Weibull modulus $(m)$ and characteristic strength $\left(\sigma_{0}\right)$ for the SiC layer are held constant throughout irradiation and are given as:

$$
m=6 \text { and } \sigma_{0}=9640000 \mathrm{~Pa}-\mathrm{m}^{3 / 6}
$$

The Weibull modulus for the $\mathrm{PyC}$ is assumed to be 9.5 , which corresponds to a density of 1.9 $\mathrm{g} / \mathrm{cm}^{3}$.

The Weibull characteristic strength of the $\mathrm{PyC}[23,24]$ is a function of anisotropy and is determined from the following equation for room temperature:

$$
\sigma_{0}=10^{6} \times\left(154.46 X^{2}-141.1 X\right)
$$

where $X$ is a fitting parameter with a default value of 1.02 . The characteristic strength has units of $\mathrm{Pa}-\mathrm{m}^{3 / 9.5}$ when the Weibull modulus is 9.5.

At other temperatures and nonzero fluence, Eq. 3.65 is multiplied by a factor as follows:

$$
\text { factor }=[(1+0.23 \phi)(1+0.00015 T)]^{1 / 2},
$$

where $\phi\left(10^{25} \mathrm{n} / \mathrm{m}^{2}, \mathrm{E}>0.18 \mathrm{MeV}\right)$ is the fast neutron fluence and $T\left({ }^{\circ} \mathrm{C}\right)$ is the temperature.

\subsubsection{BISON Benchmarked against PARFUME}

The material models were tested for a TRISO fuel particle under representative irradiation conditions given in Table 3.10. The temperatures of $700^{\circ} \mathrm{C}, 1000^{\circ} \mathrm{C}$ and $1300^{\circ} \mathrm{C}$ are chosen in order to fall within the validity range of the material properties. The fuel parameters given in Table 3.11 are based on the AGR-5/6/7 fuel specification.

For each of the three conditions in Table 3.10, the tangential stresses at the inner and outer surfaces of each layer are plotted in Fig. 3.17. The tangential stresses are tensile in the inner $\mathrm{PyC}(\mathrm{IPyC})$ and outer PyC (OPyC) layers while being compressive in the $\mathrm{SiC}$ layer. The extremum tensile stress in both PyC layers and the compressive stress in the SiC layer decrease as temperature increases due to irradiation creep. The preliminary results show good agreement between BISON and PARFUME. 


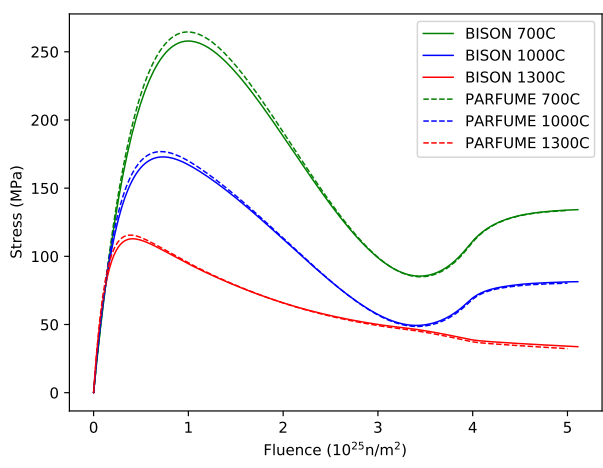

(a) Inner wall tangential stress for IPyC.

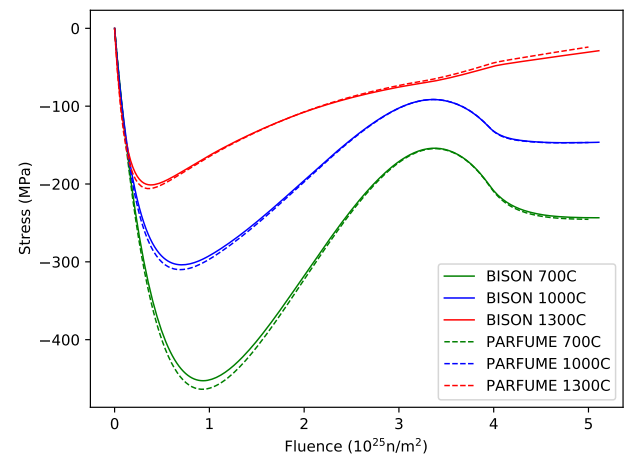

(c) Inner wall tangential stress for $\mathrm{SiC}$.

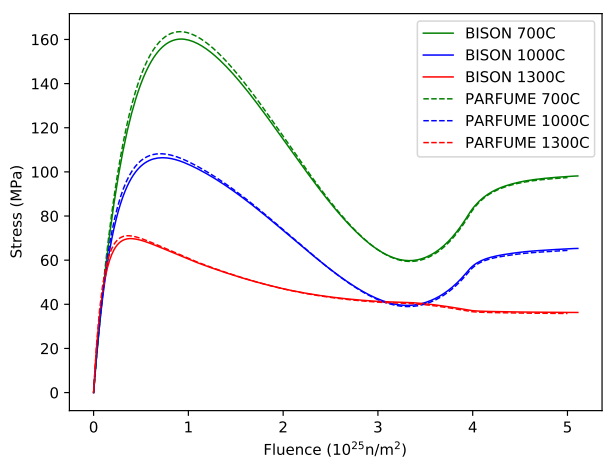

(e) Inner wall tangential stress for OPyC.

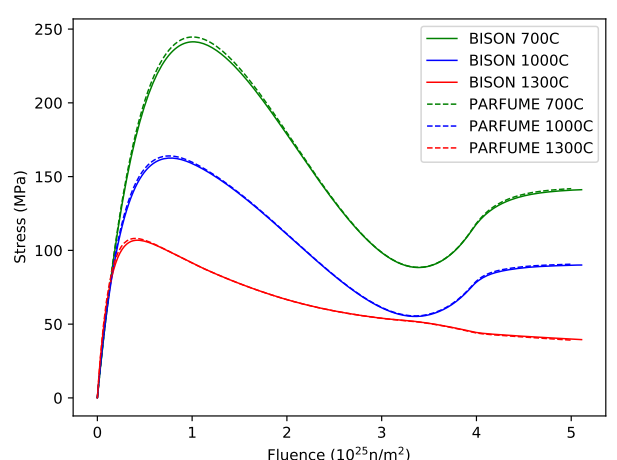

(b) Outer wall tangential stress for IPyC.

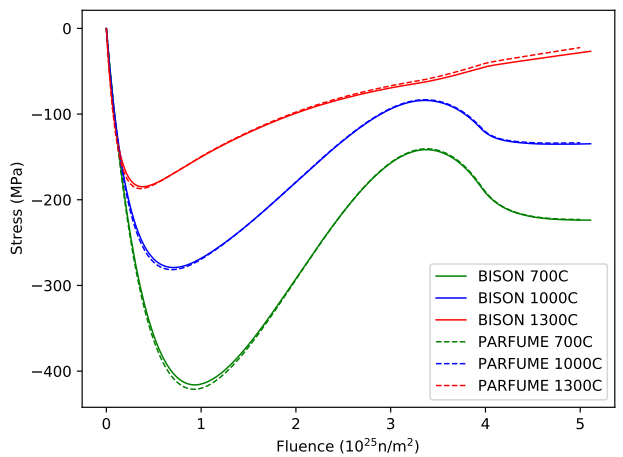

(d) Outer wall tangential stress for SiC.

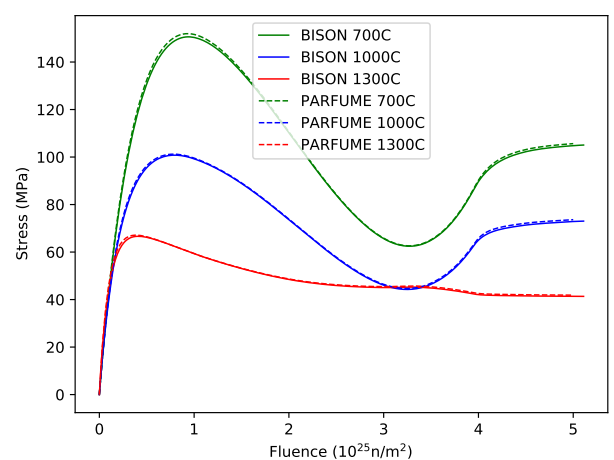

(f) Outer wall tangential stress for OPyC.

Figure 3.17: Comparison of code results. 
Table 3.10: Irradiation conditions.

\begin{tabular}{lcccc}
\hline Conditions & EFPD & $\begin{array}{c}\text { Burnup } \\
(\boldsymbol{\%} \text { FIMA })\end{array}$ & $\begin{array}{c}\text { Fast fluence } \\
\left(\times 10^{25} \mathbf{n} / \mathbf{m}^{2}\right), \mathbf{E}>\mathbf{0 . 1 8} \mathbf{M e V}\end{array}$ & $\begin{array}{c}\text { Irradiation } \\
\text { temperature }\left({ }^{\circ} \mathbf{C}\right)\end{array}$ \\
\hline 1 & 500 & 13.5 & 5 & 700 \\
2 & 500 & 13.5 & 5 & 1000 \\
3 & 500 & 13.5 & 5 & 1300 \\
\hline
\end{tabular}

Table 3.11: Fuel parameters.

\begin{tabular}{llc}
\hline Category & \multicolumn{1}{c}{ Parameter } & Nominal values \\
\hline \multirow{4}{*}{ Fuel characteristics } & ${ }^{235}$ U enrichment (wt\%) & 15.5 \\
& Carbon/uranium (atomic ratio) & 0.4 \\
& Oxygen/uranium (atomic ratio) & 1.5 \\
\hline \multirow{5}{*}{ Particle geometry } & Kernel diameter $(\mu \mathrm{m})$ & 425 \\
& Buffer thickness $(\mu \mathrm{m})$ & 100 \\
& IPyC/OPyC thickness $(\mu \mathrm{m})$ & 40 \\
& SiC thickness $(\mu \mathrm{m})$ & 35 \\
\hline \multirow{5}{*}{ Fuel properties } & Kernel density $\left(\mathrm{g} / \mathrm{cm}^{3}\right)$ & 11.0 \\
& Kernel theoretical density $\left(\mathrm{g} / \mathrm{cm}^{3}\right)$ & 11.4 \\
& Buffer density $\left(\mathrm{g} / \mathrm{cm}^{3}\right)$ & 1.05 \\
& Buffer theoretical density $\left(\mathrm{g} / \mathrm{cm}^{3}\right)$ & 2.25 \\
& IPyC density $\left(\mathrm{g} / \mathrm{cm}^{3}\right)$ & 1.90 \\
& OPyC density $\left(\mathrm{g} / \mathrm{cm}^{3}\right)$ & 1.90 \\
& IPyC/OPyC BAF & 1.05 \\
\hline
\end{tabular}

\subsection{Monte Carlo Evaluation and Statistical Failure Capabilities}

The ability of the fuel to contain fission products is largely dictated by the quality of the manufacturing process, because most fission product release is expected to occur due to failure of coating layers in a small number of particles at the locations of defects. To account for statistical variation in physical dimensions and material properties from particle to particle, a new Monte Carlo capability was developed in BISON to compute failure probability for a statistically sampled batch of particles. This enables assessment of the structural integrity of TRISO particles and its effects on the diffusion of fission products under neutron irradiation. 


\subsubsection{Failure Modes}

Outlined in the sections below are several potential TRISO fuel failure mechanisms considered in the current work.

\subsubsection{Pressure vessel failure}

Early on during irradiation, pyrocarbon layers shrink and put the SiC layer in compression. As irradiation progresses, the creep of the pyrocarbon layers tends to relax some of this compressive stress. In addition, buildup of fission gas pressure tends to put the coating layers in tension. Fig. 3.18 shows the tangential stress at the inner surface of the SiC layer under three temperature conditions. This stress value is important, as it is used to determine whether a particle fails. Since $\mathrm{CO}$ production in a UCO kernel is relatively small, gas pressure is usually not high enough to make the tangential stress in the $\mathrm{SiC}$ layer become tensile. Thus, pressure vessel failure is not likely to occur for an intact TRISO UCO kernel.

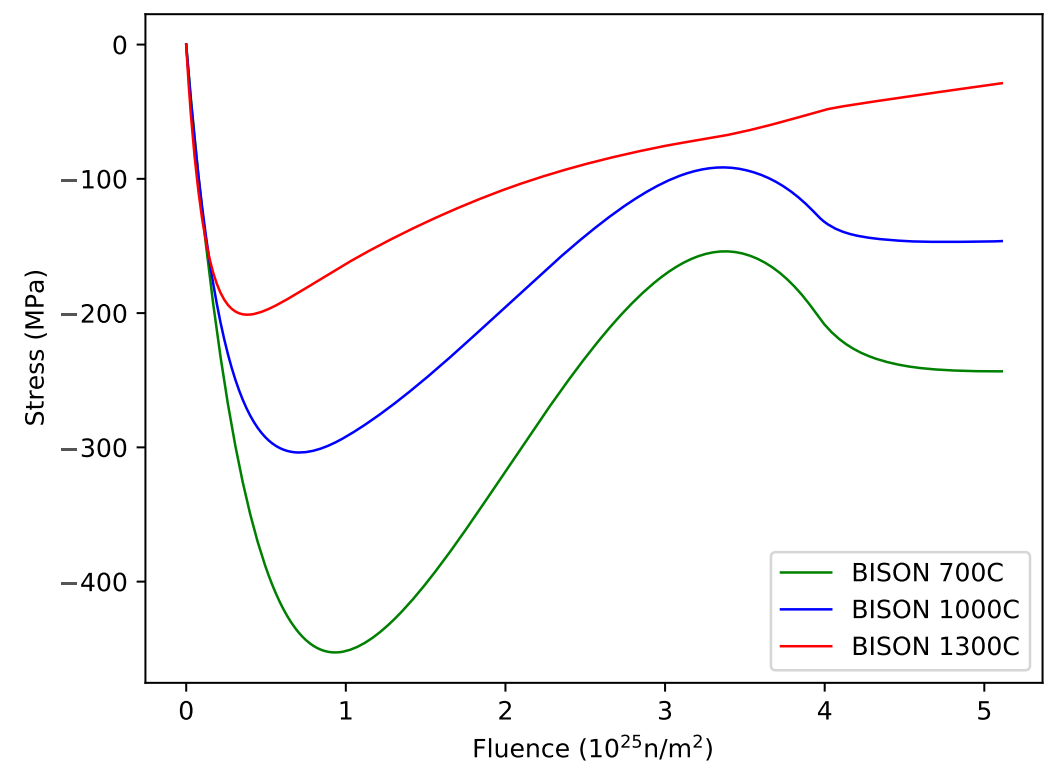

Figure 3.18: Tangential stress at the inner surface of the $\mathrm{SiC}$ layer.

\subsubsection{Cracking of IPyC}

During irradiation, shrinkage of the pyrocarbon layers causes a significant tensile stress in those layers. If stress exceeds the tensile strength of that material, a radial crack forms in that pyrocarbon layer. The radial crack leads to a high local tensile stress in the SiC layer adjacent to 
the cracked pyrocarbon layer, potentially leading to particle failure. As shown in Figs.3.19a and $3.19 \mathrm{~b}$, the stress in the $\mathrm{SiC}$ layer near the crack tip is elevated significantly due to the development of cracking in the IPyC or OPyC layer.

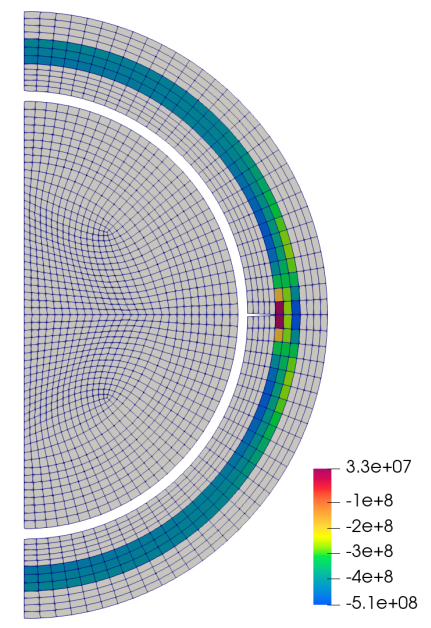

(a) Stress contour for $\mathrm{T}=700^{\circ} \mathrm{C}$ when maximum tangential stress is reached.

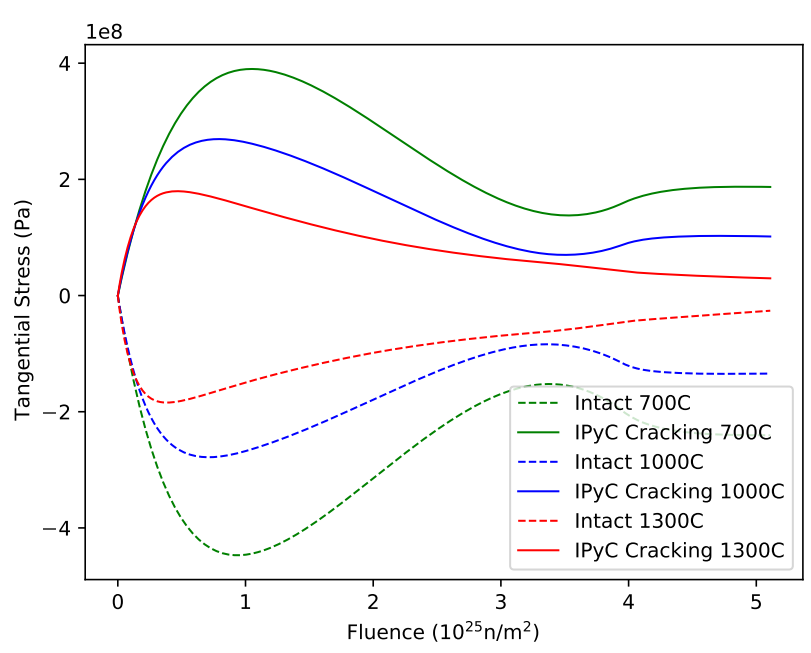

(b) Inner wall tangential stress for SiC.

Figure 3.19: Cracking of IPyC.

\subsubsection{Pressure vessel failure of an aspherical particle}

A single flat facet on one side of the particle created during fabrication is a common cause of aspherical behavior in particle fuel. The degree of asphericity for a particle is measured by the aspect ratio (see Fig. 3.20):

$$
A=\frac{2 R}{\left(R+\sqrt{R^{2}-r^{2}}\right)} .
$$

During irradiation, the faceted portion of the particle acts as a flat plate that retains the internal gas pressure. If the pressure builds up high enough, this results in a local region of tensile stress in the central portion of the plate, potentially contributing to particle failures. As shown in Fig. 3.21b, the tangential stresses in the SiC layer remain compressive through the end of irradiation because $\mathrm{CO}$ production is limited in $\mathrm{UCO}$ fuel.

\subsubsection{Weibull Failure Theory}

A Weibull failure criterion is used to determine vessel failure for the inner pyrolytic carbon (IPyC) layer, silicon carbide (SiC) layer, and outer pyrolytic carbon (OPyC) layer. The max- 


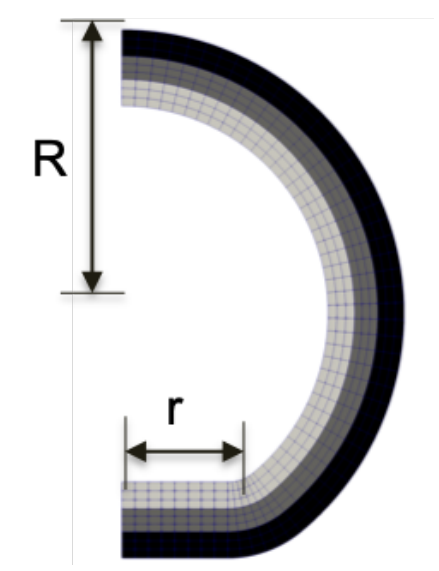

Figure 3.20: Aspect ratio of an aspherical particle.

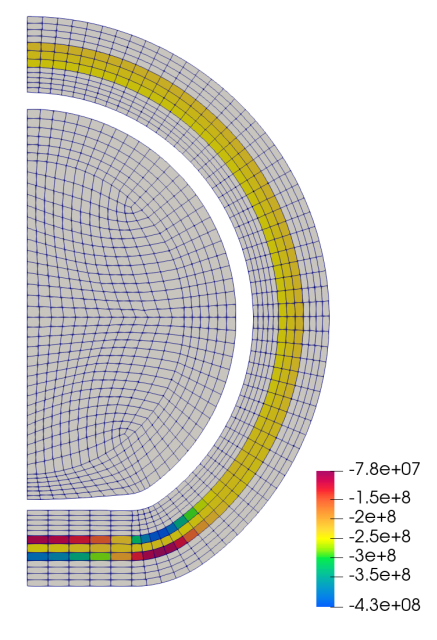

(a) Stress contour for $\mathrm{T}=700^{\circ} \mathrm{C}$ at the end of irradiation with an aspect ratio of 1.04 .

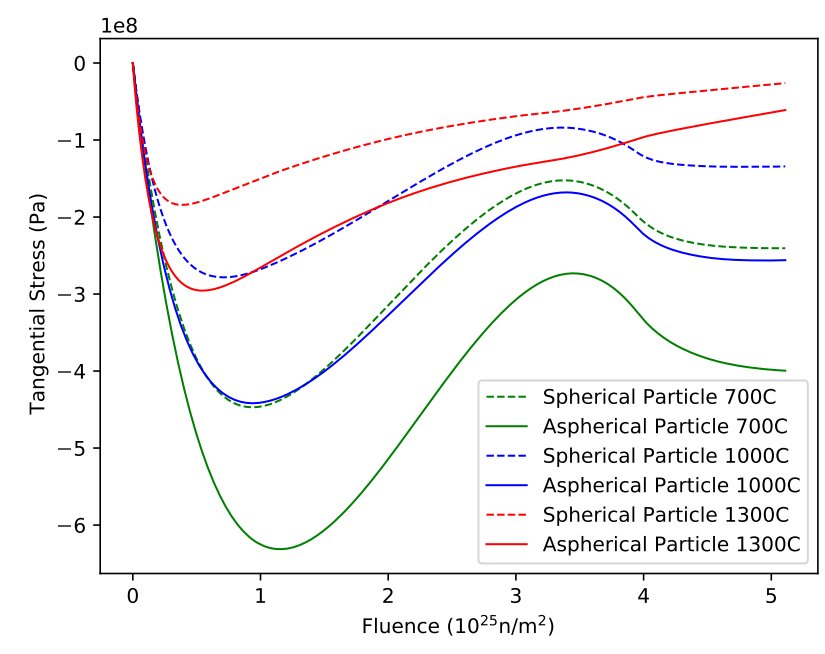

(b) $\mathrm{SiC}$ stress in the faceted portion.

Figure 3.21: Aspherical Particle.

imum stress $\left(\sigma_{c}\right)$ is compared to a strength sampled from a Weibull distribution having mean strength $\sigma_{m s}$ to determine whether failure occurs. The effective mean strength $\sigma_{m s}$ is given as:

$$
\sigma_{m s}=\frac{\sigma_{0}}{I_{n}^{\frac{1}{m}}}
$$

where $\sigma_{0}$ is the characteristic strength and $m$ is the Weibull modulus. The integral $I_{n}$ is a normalized integration of the stress distribution using the principle of independent action model as 
follows:

$$
I_{n}=\frac{\int_{V}\left(\sigma_{1}^{m}+\sigma_{2}^{m}+\sigma_{3}^{m}\right) d V}{\sigma_{c}^{m}}
$$

where $\sigma_{c}$ is the maximum value calculated for a principal stress anywhere in the volume and $\sigma_{1}$, $\sigma_{2}$, and $\sigma_{3}$ are the three principal stresses. Negative principal stresses are not included in this integral because compressive stresses do not contribute to fracture.

\subsubsection{High-Fidelity Analysis}

For computational efficiency, it is important that the fuel performance calculations are primarily conducted using inexpensive 1D models, since so many calculations are necessary for the Monte Carlo sampling procedure. However, stress concentrations due to phenomena such as cracking must be characterized using a higher-dimensionality, higher-fidelity model. To account for these multi-dimensional phenomena within a 1D fuel performance model, a high-fidelity analysis is first performed to obtain the mean strength based on the multi-dimensional stress distribution and stress correlation factor. The stress correlation factor is defined as the ratio of maximum tangential stress calculated in the multi-dimensional and one-dimensional simulations. The multidimensional strength and stress correlation factor is calculated prior to statistical modeling and used in the 1D model to determine failure. The flow diagram for this statistical approach is shown in Fig. 3.22.

\section{High-Fidelity Analysis}

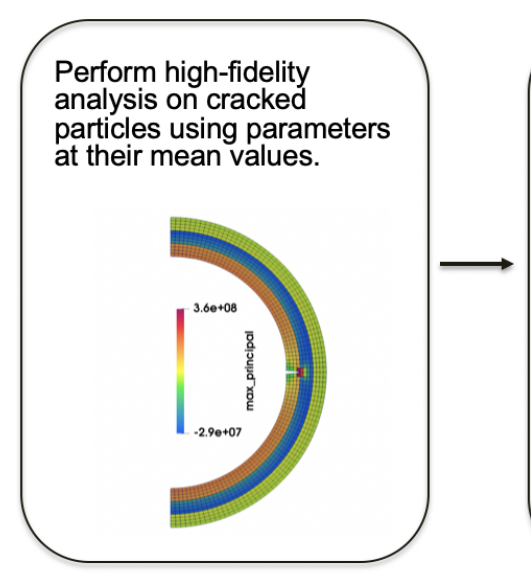

Stress Correlation

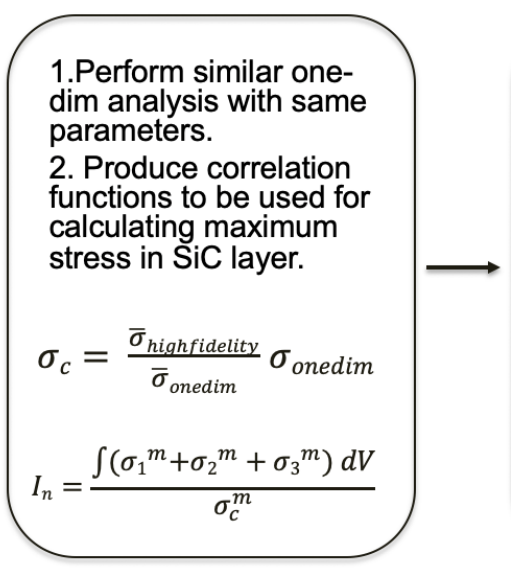

Failure Probability Determination

\section{Perform 1D} simulation to calculate stresses in Monte Carlo sampling.

2. Use Weibull statistics to determine failure probability for particles.

Figure 3.22: High-fidelity analysis and stress correlation. 


\subsubsection{Stress correlation for IPyC cracking}

The maximum tangential stress histories in the $\mathrm{SiC}$ layer for a cracked particle (near the crack tip) and an intact particle are shown in Fig. 3.23. The maximum SiC stress in a cracked particle is approximated as:

$$
\sigma_{\text {correlation }}=-\frac{\bar{\sigma}_{2 \mathrm{D}}}{\bar{\sigma}_{1 \mathrm{D}}} \sigma_{1 \mathrm{D}}
$$

where $\bar{\sigma}_{2 \mathrm{D}}$ and $\bar{\sigma}_{1 \mathrm{D}}$ are the maximum stresses calculated in the 2D and 1D analyses at the mean values for a specified batch of particles. The negative sign reflects the change of tangential stress from compressive to tensile. Upon varying statistical parameters, the maximum stress in the $\mathrm{SiC}$ layer is determined from the 1D FE solution for $\sigma_{1 \mathrm{D}}$.

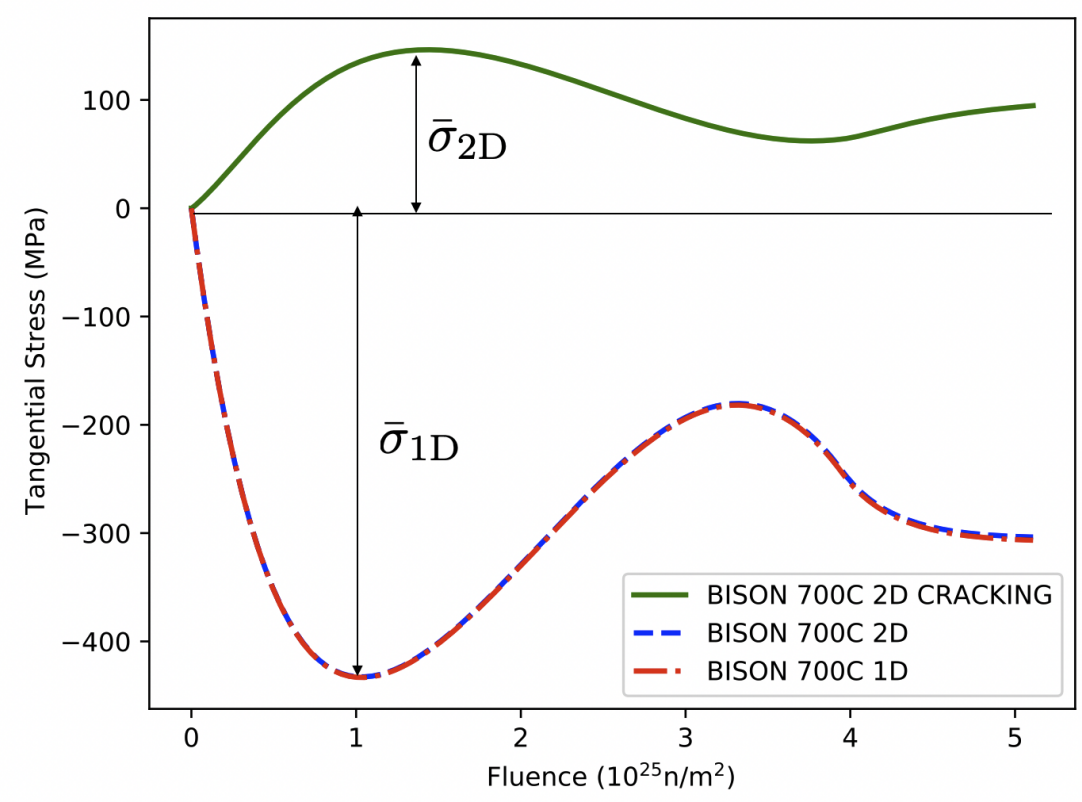

Figure 3.23: Stress correlation for IPyC cracking.

\subsubsection{Stress correlation for aspherical particle}

The tangential stress histories for a representative faceted and spherical fuel particle are shown in Fig. 3.24. In evaluating asphericity, a second term is added to correctly estimate the maximum stress $\left(\sigma_{c}\right)$ for an aspherical particle:

$$
\sigma_{\text {correlation }}=\frac{\bar{\sigma}_{2 \mathrm{D}}}{\bar{\sigma}_{1 \mathrm{D}}} \sigma_{1 \mathrm{D}-\mathrm{min}}+\frac{\Delta \bar{\sigma}_{2 \mathrm{D}}}{\Delta \bar{\sigma}_{1 \mathrm{D}}} \Delta \sigma_{1 \mathrm{D}}
$$


where $\Delta \bar{\sigma}_{2 \mathrm{D}}, \Delta \bar{\sigma}_{1 \mathrm{D}}$, and $\Delta \sigma_{1 \mathrm{D}-\mathrm{min}}$ are changes in the stresses $\bar{\sigma}_{2 \mathrm{D}}, \bar{\sigma}_{1 \mathrm{D}}$, and $\sigma_{1 \mathrm{D}-\mathrm{min}}$, respectively, going from the minimum to the end of irradiation. If a second extremum (or maximum) occurs before the end of irradiation, $\Delta \bar{\sigma}_{2 \mathrm{D}}, \Delta \bar{\sigma}_{1 \mathrm{D}}$, and $\Delta \sigma_{1 \mathrm{D}}$ are taken as changes in these stress going from the minimum to the maximum. The additional term is needed for asphericity evaluations, because failures due to asphericity occur after the first extremum for $\sigma_{1 \mathrm{D}}$ is reached, when shrinkage effects from the pyrocarbons are diminishing.

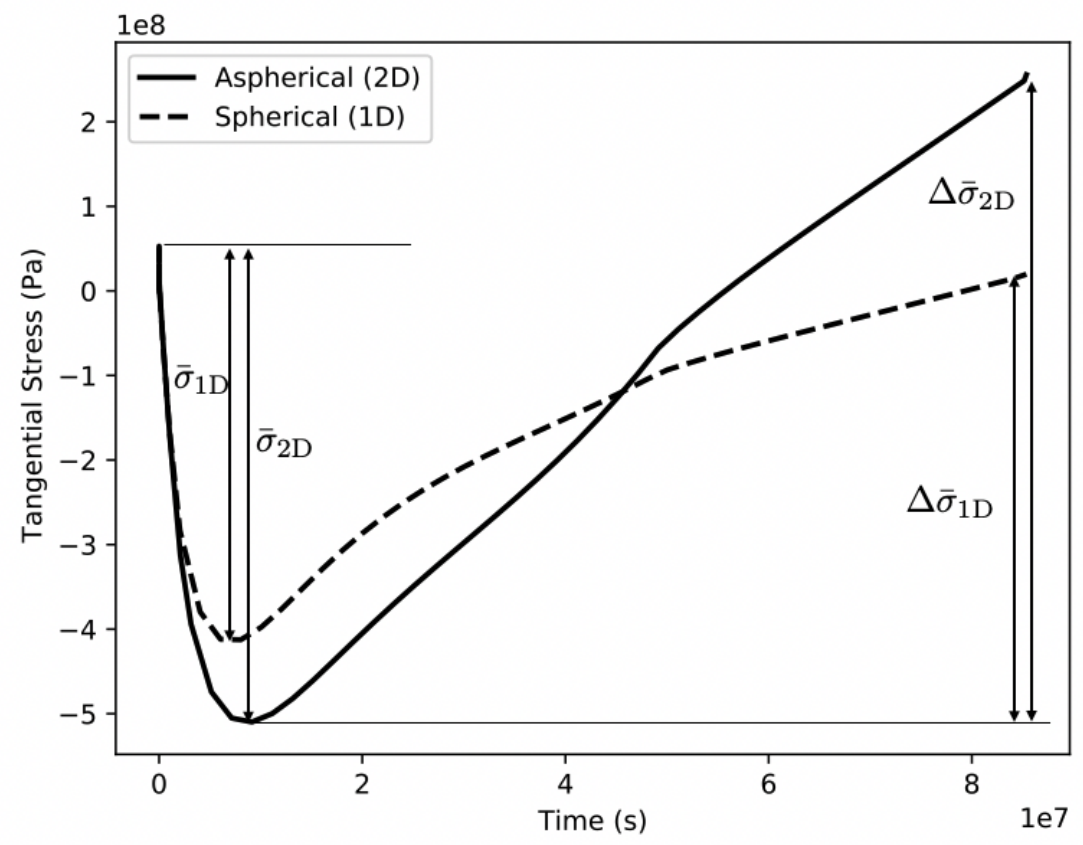

Figure 3.24: Stress correlation for an aspherical particle.

\subsubsection{Preliminary Failure Probability Calculation}

The failure probability for a TRISO UCO fuel particle under the irradiation conditions given in Table 3.10 was calculated using a Monte Carlo scheme. The statistical variation of particle dimensions and material properties is listed in Table 3.12. The stress correlation functions and effective mean strength are precomputed for each condition, as given in Table 3.13. BISON and PARFUME are used to compute the probability of IPyC layer cracking and $\mathrm{SiC}$ failure. Both codes use the same stress correlation factors and effective mean strength listed in Table 3.13. Results are reported in Table 3.14, and they show good—but not excellent—agreement. The slight discrepancy in computed failure probability are possibly due to different numerical approaches and implementation in the two codes.

It is worthwhile to mention that the $\mathrm{SiC}$ failure probability of Condition 3 is very low, such that the required number of Monte Carlo samples is beyond BISON's current computational limit. A 
more efficient approach to calculate low failure probability will be developed in the future.

Table 3.12: Statistical variation of fuel parameters.

\begin{tabular}{llc}
\hline Category & Parameter & $\begin{array}{c}\text { Nominal values } \\
\pm \text { Standard Deviation }\end{array}$ \\
\hline \multirow{5}{*}{ Particle geometry } & Kernel diameter $(\mu \mathrm{m})$ & $425 \pm 10$ \\
& Buffer thickness $(\mu \mathrm{m})$ & $100 \pm 10$ \\
& IPyC/OPyC thickness $(\mu \mathrm{m})$ & $40 \pm 3$ \\
& SiC thickness $(\mu \mathrm{m})$ & $35 \pm 2$ \\
& Particle asphericity $(\mathrm{SiC}$ aspect ratio) & 1.04 \\
\hline \multirow{3}{*}{ Fuel properties } & IPyC density $\left(\mathrm{g} / \mathrm{cm}^{3}\right)$ & $1.90 \pm 0.02$ \\
& OPyC density $\left(\mathrm{g} / \mathrm{cm}^{3}\right)$ & $1.90 \pm 0.02$ \\
& IPyC/OPyC BAF & $1.05 \pm 0.005$ \\
\hline
\end{tabular}

Table 3.13: Stress correlation factors and effective mean strength.

\begin{tabular}{ccccccc}
\hline \multirow{2}{*}{ Conditions } & Intact & \multicolumn{2}{c}{ IPyC cracking } & \multicolumn{3}{c}{ Asphericity } \\
\cline { 2 - 7 } & $\sigma_{\mathrm{ms}}(\mathrm{MPa})$ & $\sigma_{\mathrm{ms}}(\mathrm{MPa})$ & $-\frac{\overline{\boldsymbol{\sigma}}_{2 \mathrm{D}}}{\bar{\sigma}_{1 \mathrm{D}}}$ & $\sigma_{\mathrm{ms}}(\mathrm{MPa})$ & $\frac{\overline{\boldsymbol{\sigma}}_{2 \mathrm{D}}}{\bar{\sigma}_{1 \mathrm{D}}}$ & $\frac{\Delta \overline{\boldsymbol{\sigma}}_{2 \mathrm{D}}}{\Delta \overline{\boldsymbol{\sigma}}_{1 \mathrm{D}}}$ \\
\hline 1 & 711 & 1198 & -0.87 & 993 & 1.41 & 1.22 \\
2 & 719 & 1198 & -0.97 & 1041 & 1.59 & 1.41 \\
3 & 719 & 1198 & -0.97 & 1095 & 1.60 & 1.48 \\
\hline
\end{tabular}

Table 3.14: Failure probability.

\begin{tabular}{ccccc}
\hline \multirow{2}{*}{ Conditions } & \multicolumn{2}{c}{ BISON } & \multicolumn{2}{c}{ PARFUME } \\
\cline { 2 - 5 } & IPyC cracking & SiC failure & IPyC cracking & SiC failure \\
\hline 1 & $9.11 \times 10^{-1}$ & $0.75 \times 10^{-3}$ & $8.94 \times 10^{-1}$ & $1.03 \times 10^{-3}$ \\
2 & $7.10 \times 10^{-2}$ & $6.10 \times 10^{-6}$ & $5.48 \times 10^{-2}$ & $7.80 \times 10^{-6}$ \\
3 & $1.01 \times 10^{-3}$ & N/A & $1.16 \times 10^{-3}$ & $1.64 \times 10^{-8}$ \\
\hline
\end{tabular}




\section{Verification}

Verification is the software development process for assuring that the code contains no programming errors and that it computes correct solutions for carefully designed problems.

This chapter discusses results for two approaches for verifying TRISO modeling capabilities in BISON. The first, strictly speaking, is better characterized as code-to-code benchmarking than verification. In this discussion, a comparison is made between BISON results and results from other codes. The second approach is more mathematically rigorous and meets the expectations of true verification. That discussion includes a demonstration of computing the correct solution to a prescribed problem using a series of more refined FE meshes.

\subsection{Code Comparison: CRP-6}

\subsubsection{Previously Run Fuel Performance Cases: 1-8, 10, 11}

The IAEA-sponsored CRP-6 explored the modeling of TRISO fuel [1]. One outcome of the work was a set of fuel performance benchmark cases. Three of these were simple enough to have analytical solutions using elastic material models. The others were more complex, using nonlinear material models and increasingly realistic boundary conditions. Several of these cases were originally run with BISON as part of [19]. Here, we present those results, along with results using the current version of BISON. Small differences are seen in the results between the original BISON calculations and the current calculations due to minor changes in modeling practices. All current results reported here use one-dimensional FEs with spherical symmetry. Details on all the cases are available in $[1,19]$.

The first three cases, as mentioned, have simple elastic material models. Results are shown in Table 4.1. The results match the analytical values very well.

Cases 4a, 4b, 4c, 4d, 5, 6, and 7 involve IPyC and SiC layers. Results are in Table 4.2. In every case, the results are within the ranges of values reported by the CRP- 6 participants. As noted in [19], values in the ranges were extracted from plots in [1].

For Case 8, the particle temperature was ramped between 873 and $1273 \mathrm{~K}$, a total of ten times. Results from this analysis are in Fig. 4.1. The BISON results match those of other codes extremely well.

The final two cases in the CRP-6 set originally run with BISON are Cases 10 and 11 . These cases are quite different from the cases already discussed. For Cases 10 and 11, the particle's 
Table 4.1: Comparison of analytical solution values for maximum tangential stress (MPa) to original (BISON13) and current BISON results for Cases 1-3.

\begin{tabular}{llrrrrr}
\hline Case & Layer & Analytical & BISON13 & Error (\%) & BISON & Error (\%) \\
\hline 1 & SiC & 125.190 & 125.230 & 0.032 & 125.130 & 0.048 \\
2 & IPyC & 50.200 & 50.287 & 0.173 & 50.176 & 0.048 \\
3 & IPyC & 8.800 & 8.700 & 1.140 & 8.700 & 1.140 \\
& SiC & 104.400 & 104.500 & 0.100 & 104.500 & 0.100 \\
\hline
\end{tabular}

Table 4.2: Comparison of the range of values reported by CRP-6 participants to original (BISON13) and current BISON results for Cases $4 \mathrm{a}-7$.

\begin{tabular}{|c|c|c|c|c|c|}
\hline Case & Layer & $\begin{array}{r}\text { CRP-6 codes } \\
\text { [range] }\end{array}$ & BISON13 & BISON & $\begin{array}{r}\text { Pictorial representation } \\
(\bullet \text { for BISON13; } \bullet \text { for BISON) }\end{array}$ \\
\hline \multirow[t]{2}{*}{$4 \mathrm{a}$} & IPyC & {$[925,970]$} & 928.0 & 925.0 & -1 \\
\hline & $\mathrm{SiC}$ & {$[-850,-775]$} & -819.0 & -816.0 & \\
\hline \multirow[t]{2}{*}{$4 b$} & IPyC & {$[-25,-25]$} & -25.0 & -25.0 & \\
\hline & $\mathrm{SiC}$ & {$[138,142]$} & 139.0 & 139.0 & ⺊ \\
\hline \multirow[t]{2}{*}{$4 \mathrm{c}$} & IPyC & {$[25,27]$} & 26.0 & 25.8 & -1 \\
\hline & $\mathrm{SiC}$ & {$[83,92]$} & 89.4 & 89.2 & $\longmapsto$ \\
\hline \multirow[t]{2}{*}{$4 d$} & IPyC & {$[25,35]$} & 27.8 & 30.3 & $\longmapsto$ \\
\hline & $\mathrm{SiC}$ & {$[71,88]$} & 87.0 & 84.5 & $\longrightarrow 1$ \\
\hline \multirow[t]{2}{*}{5} & IPyC & {$[40,58]$} & 41.9 & 44.2 & $\mapsto$ \\
\hline & $\mathrm{SiC}$ & {$[-56,-28]$} & -32.2 & -32.1 & $\longmapsto$ \\
\hline \multirow[t]{2}{*}{6} & IPyC & {$[27,38]$} & 29.2 & 31.4 & $\longmapsto \longmapsto 1$ \\
\hline & $\mathrm{SiC}$ & {$[28,48]$} & 44.9 & 41.9 & $\because-1$ \\
\hline \multirow[t]{2}{*}{7} & IPyC & {$[37,50]$} & 38.0 & 42.5 & $1 \bullet$ \\
\hline & $\mathrm{SiC}$ & {$[10,25]$} & 24.6 & 21.7 & $\longrightarrow-9$ \\
\hline
\end{tabular}

internal pressure was set by the FGR and CO-production models in the individual codes. Since different codes used different models for FGR and CO production, the codes produced fairly different results. Results for gas pressure and tangential stress in the SiC layer are in Fig. 4.2. The BISON results compare well to the range of results from other codes. 


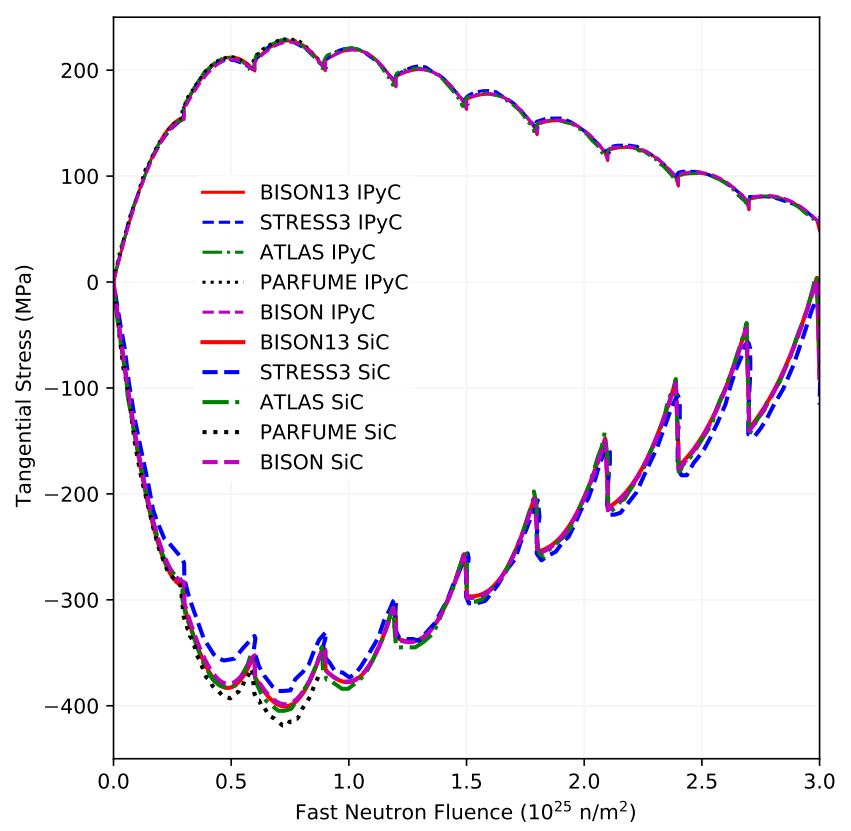

Figure 4.1: Comparison of code results for inner-wall tangential stress in IPyC and $\mathrm{SiC}$ for Case 8. Both the original (BISON13) and current BISON results match those of other codes very well.

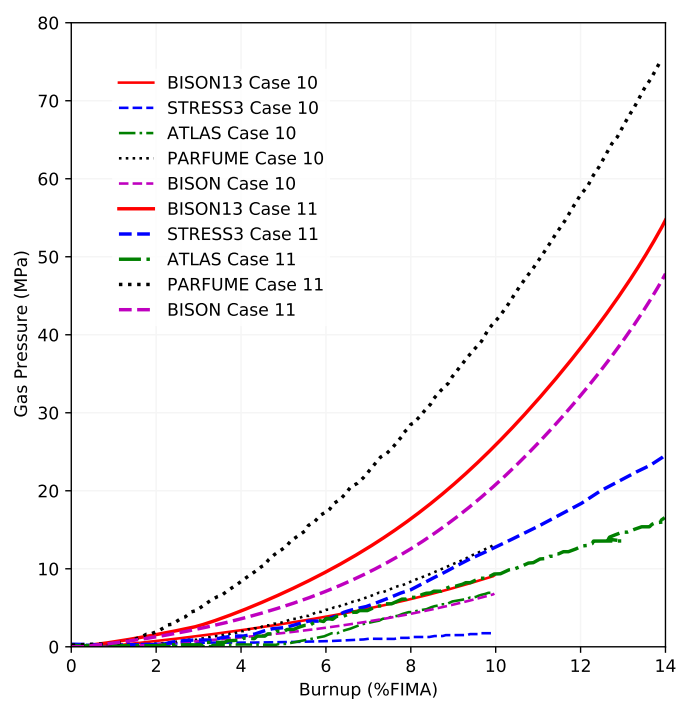

(a) Comparison of code results for gas pressure for Cases 10 and 11.

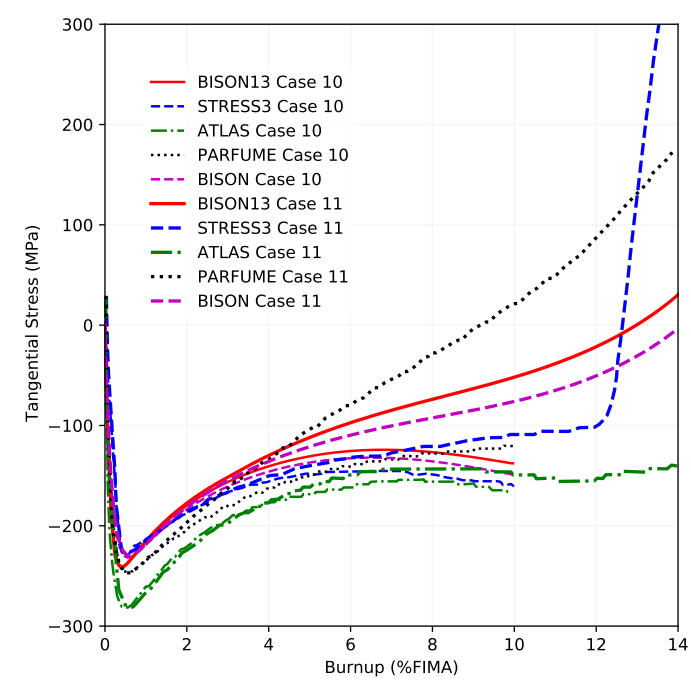

(b) Comparison of code results for inner-wall tangential stress in $\mathrm{SiC}$ for Cases 10 and 11.

Figure 4.2: Results for Cases 10 and 11. Both the original (BISON13) and current BISON results are within the range of results from other codes. 


\subsubsection{New Fuel Performance Cases: 9, 12, 13}

The remaining fuel performance cases of CRP-6-Cases 9, 12, and 13-are similar to Cases 10 and 11. For these three cases, the mean particle geometry, burnup level, and fluence level are prescribed, along with material properties. FGR and CO production are left to the analysis teams. Given the differences in those models, differences in gas pressure and tangential stress in the $\mathrm{SiC}$ layer are expected.

Case 9 has a fuel radius of $272 \mu \mathrm{m}$, with 89 days of irradiation to reach 4.79\% FIMA and a fluence of $2.1 \times 10^{25} \mathrm{n} / \mathrm{m}^{2}$. Results are shown in Fig. 4.3. The BISON results are comparable to those of other codes.

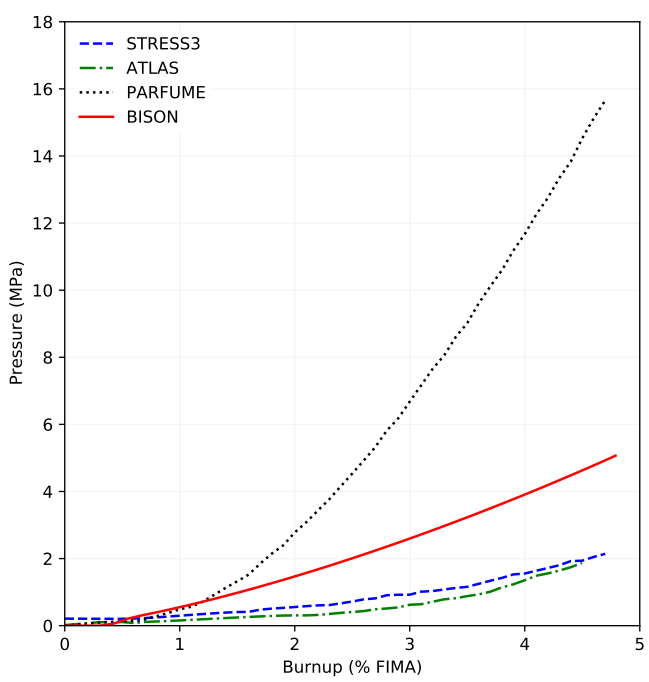

(a) Internal pressure for Case 9.

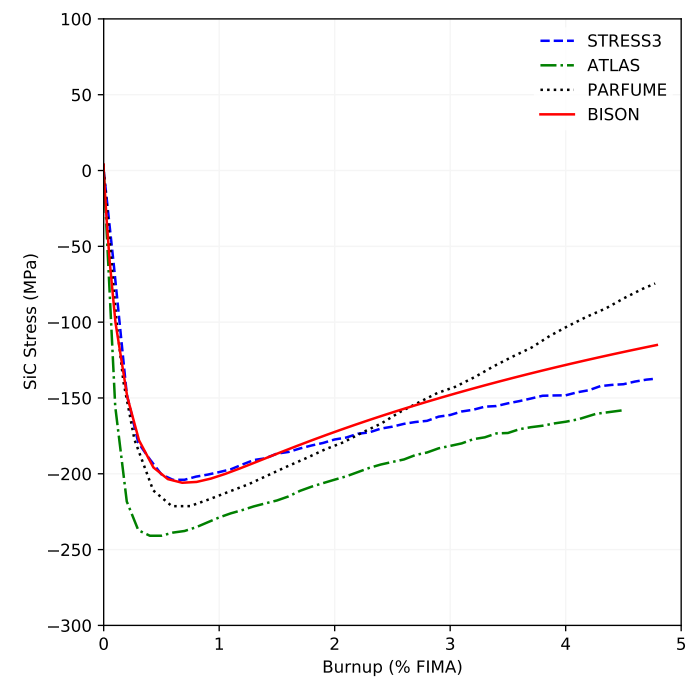

(b) Tangential stress in the $\mathrm{SiC}$ layer for Case 9.

Figure 4.3: Results for Case 9. Results from other codes extracted from [1].

Case 12 is somewhat unique among the CRP-6 benchmark cases in that (1) its fuel is UCO rather than $\mathrm{UO}_{2}$ and (2) the fuel is highly enriched. The fuel radius is $100 \mu \mathrm{m}$ and reaches $79 \%$ FIMA and a fluence of $3.8 \times 10^{25} \mathrm{n} / \mathrm{m}^{2}$ in 170 days of irradiation. Results are shown in Fig. 4.4. The BISON results are comparable to those of other codes.

Case 13 has a fuel radius of $251 \mu \mathrm{m}$ and reaches $20 \%$ FIMA and a fluence of $5.4 \times 10^{25} \mathrm{n} / \mathrm{m}^{2}$ in 600 days of irradiation. Results are shown in Fig. 4.5. The BISON results are comparable to those of other codes.

For all of the last 5 cases, one metric for comparison is the fraction of failed particles with increasing burnup. That comparison has not yet been made and is left as a future investigation. 


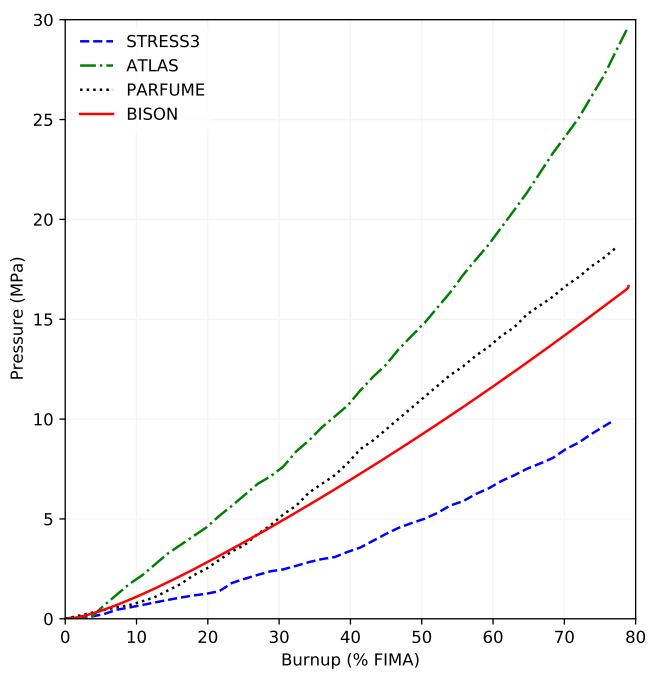

(a) Internal pressure for Case 12.

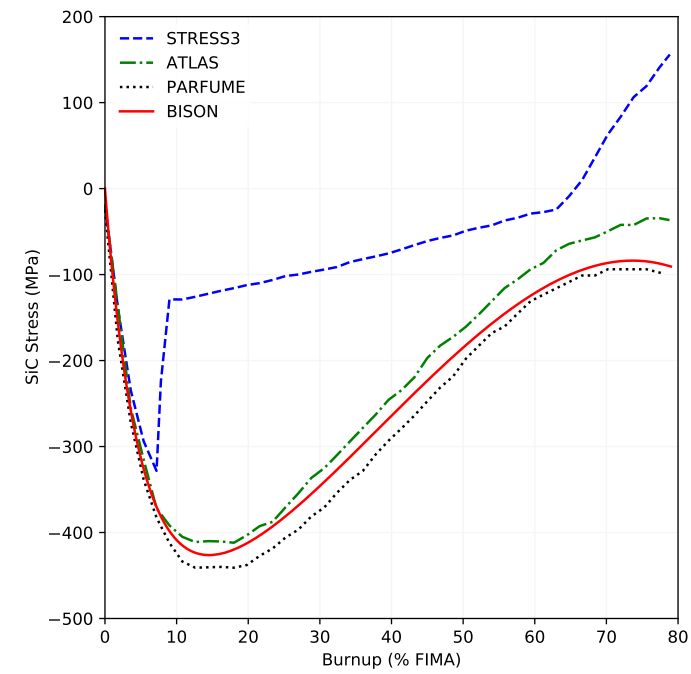

(b) Tangential stress in the SiC layer for Case 12.

Figure 4.4: Results for Case 12. Results from other codes extracted from [1].

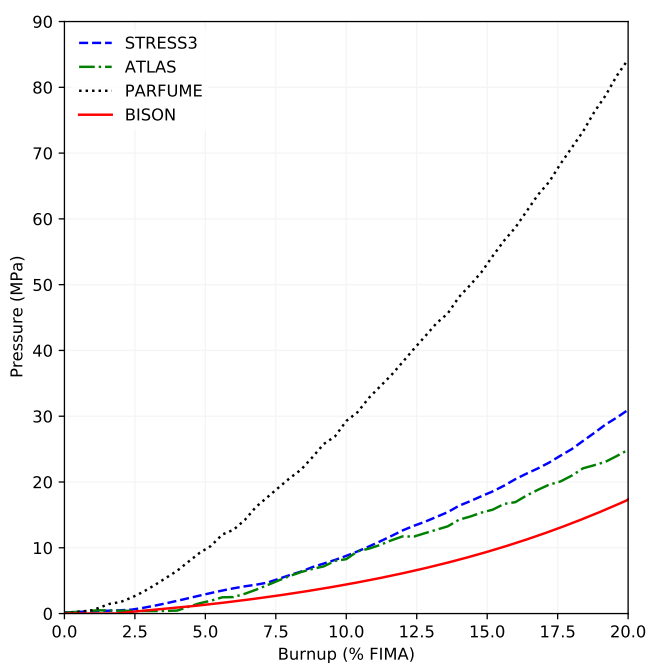

(a) Internal pressure for Case 13 .

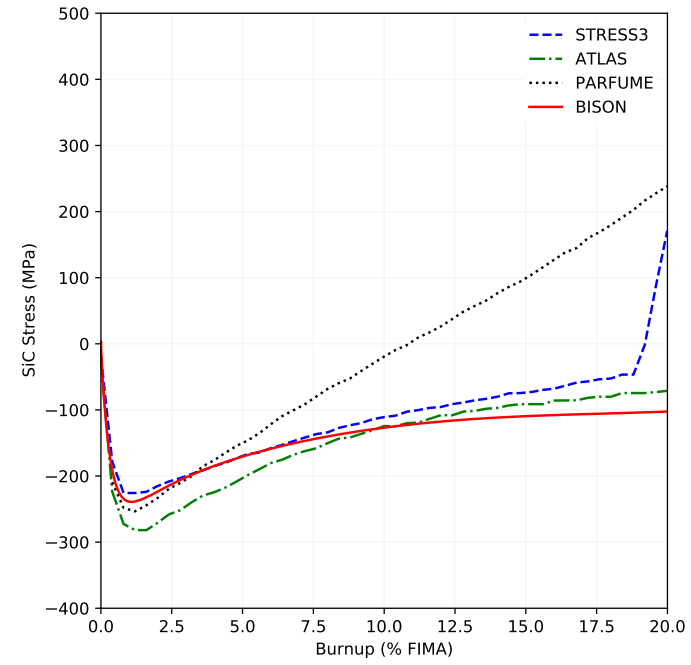

(b) Tangential stress in the $\mathrm{SiC}$ layer for Case 13.

Figure 4.5: Results for Case 13. Results from other codes extracted from [1].

\subsubsection{New Diffusion Cases: 1-5}

CRP-6 also includes a set of cases intended to exercise diffusion capabilities. These cases included a set based on assumed model parameters, along with a set based on heating tests. The first set, Cases 1 through 5, have been evaluated with BISON. For these cases, diffusion of one or more fission products is measured via the fractional release. 
Cases $1 \mathrm{a}$ and $1 \mathrm{~b}$ are for the diffusion of pre-existing cesium in a bare kernel. Case 1a has a temperature of $1200^{\circ} \mathrm{C}$, and Case $1 \mathrm{~b}$ has a temperature of $1600^{\circ} \mathrm{C}$. The simulation time is 200 hours for both cases. Results are shown in Table 4.3.

Table 4.3: Comparison of the range of values reported by CRP-6 participants to BISON results for Case 1, fractional release of cesium from a bare kernel.

\begin{tabular}{lrrr}
\hline Case & $\begin{array}{r}\text { CRP-6 codes } \\
\text { [range] }\end{array}$ & BISON & $\begin{array}{r}\text { Pictorial representation } \\
(\bullet \text { for BISON })\end{array}$ \\
\hline $1 \mathrm{a}$ & {$[0.453,0.498]$} & 0.466 & $\longmapsto$ \\
$1 \mathrm{~b}$ & {$[0.970,1.000]$} & 1.000 & $\longmapsto$ \\
\hline
\end{tabular}

Cases $2 \mathrm{a}$ and $2 \mathrm{~b}$ are similar to $1 \mathrm{a}$ and $1 \mathrm{~b}$ except that the particle includes the kernel, buffer, and inner PyC. Results are in Table 4.4.

Table 4.4: Comparison of the range of values reported by CRP-6 participants to BISON results for Case 2, fractional release of cesium from the kernel, buffer, and inner PyC.

\begin{tabular}{lrrr}
\hline Case & $\begin{array}{r}\text { CRP-6 codes } \\
\text { [range] }\end{array}$ & BISON & $\begin{array}{r}\text { Pictorial representation } \\
(\bullet \text { for BISON) }\end{array}$ \\
\hline $2 \mathrm{a}$ & {$[0.006,0.030]$} & 0.027 & 1 \\
$2 \mathrm{~b}$ & {$[0.968,0.996]$} & 0.995 & 1 \\
\hline
\end{tabular}

Similar to Cases 1 and 2, Case 3 evaluates diffusion of pre-existing fission products. Case 3, however, involves a complete TRISO particle. In Case 3a, the temperature is $1600^{\circ} \mathrm{C}$ held for 200 hours. In Case $3 \mathrm{~b}$, the temperature is $1800^{\circ} \mathrm{C}$. In Case $3 \mathrm{c}$, the temperature is $1600^{\circ} \mathrm{C}$ for 200 hours, followed by a temperature of $1800^{\circ} \mathrm{C}$ for 200 hours.

Cases $3 \mathrm{~d}$ and $3 \mathrm{e}$ have the same temperature conditions as Case 3c. In Case $3 \mathrm{~d}$, the $\mathrm{SiC}$ layer cracks at $1800^{\circ} \mathrm{C}$. In Case $3 \mathrm{e}$, the $\mathrm{SiC}$ layer cracks at $1600^{\circ} \mathrm{C}$, and the inner and outer PyC layers crack at $1800^{\circ} \mathrm{C}$. Cracked layers have their diffusivities set to $1 \times 10^{-6}$.

Results for Case 3 are in Table 4.5.

Case 4 does not involve a pre-existing inventory of fission products. Instead, fission products are built up over 500 days of irradiation at $1000^{\circ} \mathrm{C}$, reaching a burnup of $10 \%$ FIMA and a fast neutron fluence of $2 \times 10^{25} \mathrm{n} / \mathrm{m}^{2}$. Case $4 \mathrm{a}$ ends with 200 hours at $1600^{\circ} \mathrm{C}$, and Case $4 \mathrm{~b}$ ends with 200 hours at $1800^{\circ} \mathrm{C}$. Case $4 \mathrm{c}$ has 200 hours at $1600^{\circ} \mathrm{C}$, followed by 200 hours at $1800^{\circ} \mathrm{C}$. Case $4 \mathrm{~d}$ is much like Case $4 \mathrm{c}$, but the $\mathrm{SiC}$ layer cracks at $1600^{\circ} \mathrm{C}$, and the inner and outer $\mathrm{PyC}$ layers crack at $1800^{\circ} \mathrm{C}$. Cracked layers have their diffusivities set to $1 \times 10^{-6}$.

Results for Case 4 are in Table 4.6. 
Table 4.5: Comparison of the range of values reported by CRP-6 participants to BISON results for Case 3, fractional release of cesium from the TRISO particle.

\begin{tabular}{lrrr}
\hline Case & $\begin{array}{r}\text { CRP-6 codes } \\
{[\text { range }]}\end{array}$ & BISON & $\begin{array}{r}\text { Pictorial representation } \\
(\bullet \text { for BISON })\end{array}$ \\
\hline $3 \mathrm{a}$ & {$\left[6.59 \times 10^{-5}, 1.13 \times 10^{-3}\right]$} & $1.33 \times 10^{-4}$ & \\
$3 \mathrm{~b}$ & {$[0.203,0.218]$} & 0.210 & $\longmapsto$ \\
$3 \mathrm{c}$ & {$[0.220,0.239]$} & 0.225 & $\longmapsto$ \\
$3 \mathrm{~d}$ & {$[0.999,1.000]$} & 1.000 & $\longmapsto$ \\
$3 \mathrm{e}$ (cesium) & {$[0.970,1.000]$} & 1.000 & $\longmapsto$ \\
$3 \mathrm{e}$ (gases) & {$[0.980,1.000]$} & 1.000 & $\longmapsto$ \\
\hline
\end{tabular}

Table 4.6: Comparison of the range of values reported by CRP-6 participants to BISON results for Case 4, fractional release of cesium and silver from the TRISO particle with 500 days of irradiation.

\begin{tabular}{llrrr}
\hline Case & $\begin{array}{r}\text { CRP-6 codes } \\
\text { [range] }\end{array}$ & BISON & $\begin{array}{r}\text { Pictorial representation } \\
(\bullet \text { for BISON) }\end{array}$ \\
\hline Cesium & $4 \mathrm{a}$ & {$\left[1.64 \times 10^{-4}, 1.47 \times 10^{-3}\right]$} & $2.44 \times 10^{-4}$ & \\
& $4 \mathrm{~b}$ & {$[0.20,0.23]$} & 0.20 & \\
& $4 \mathrm{c}$ & {$[0.21,0.24]$} & 0.22 & \\
& $4 \mathrm{~d}$ & {$[1.00,1.00]$} & 1.00 & \\
\hline \multirow{2}{*}{ Silver } & $4 \mathrm{a}$ & {$[0.27,0.55]$} & 0.38 & \\
& $4 \mathrm{~b}$ & {$[0.58,0.95]$} & 0.78 & \\
& $4 \mathrm{c}$ & {$[0.92,0.98]$} & 0.90 & \\
\hline
\end{tabular}

Like Case 4, Case 5 builds up fission products over a period of irradiation. In Case 5, the irradiation period is 1,000 days. That time is divided into 10 cycles, during each of which the temperature ramps up from 600 to $1000^{\circ} \mathrm{C}$. The final burnup is $10 \%$ FIMA, and the final fast neutron fluence is $2 \times 10^{25} \mathrm{n} / \mathrm{m}^{2}$. Case 5 a considers cesium and silver diffusion after 1,000 days, while Case $5 \mathrm{~b}$ adds 200 hours at $1600^{\circ} \mathrm{C}$.

Results are in Table 4.7.

As demonstrated by these results, BISON's predictions of fission product diffusion in TRISO fuel particles compare very well to those of other codes, providing evidence that BISON's dif- 
Table 4.7: Comparison of the range of values reported by CRP-6 participants to BISON results for Case 5, fractional release of cesium and silver from TRISO particle with 10 cycles of irradiation.

\begin{tabular}{lcrrr}
\hline Case & $\begin{array}{r}\text { CRP-6 codes } \\
\text { [range] }\end{array}$ & BISON & $\begin{array}{r}\text { Pictorial representation } \\
(\bullet \text { for BISON })\end{array}$ \\
\hline Cesium & $5 \mathrm{a}$ & {$\left[2.19 \times 10^{-19}, 1.92 \times 10^{-11}\right]$} & $2.45 \times 10^{-18}$ & \\
& $5 \mathrm{~b}$ & {$\left[3.07 \times 10^{-4}, 1.22 \times 10^{-3}\right]$} & $1.49 \times 10^{-4}$ & $\bullet$ \\
\hline Silver & $5 \mathrm{a}$ & {$\left[5.55 \times 10^{-6}, 5.06 \times 10^{-5}\right]$} & $6.51 \times 10^{-6}$ & \\
& $5 \mathrm{~b}$ & {$[0.14,0.54]$} & 0.40 & \\
\hline
\end{tabular}

fusion calculations meet expectations.

The CRP-6 benchmark cases have been extremely valuable as a means of exercising BISON's TRISO capabilities and determining best practices for particle analysis. That BISON's results match published results so well, for both stress and diffusion, indicates that the relevant equations were coded correctly.

\subsection{Code Comparison: AGR-5/6/7 Irradiation Test Predictions}

DOE's Advanced Gas Reactor program planned a series of irradiation experiments on TRISO fuel. The AGR-5/6/7 experiments test TRISO particles with low-enriched UCO fuel. The AGR5/6/7 test train houses 5 capsules, with each capsule containing compacts filled with TRISO particles. The TRISO particle geometry and material properties are listed in Table 4.8. The details of these experiments can be found in [38].

Failure probability calculations were performed on three compacts in Capsule 5. Their irradiation conditions are listed in Table 4.9. For these compacts, the inner-wall tangential stresses in $\mathrm{SiC}$ of an intact particle are plotted in Fig. 4.6. The plots show good agreement between BISON and PARFUME. The evolution of the buffer outer and IPyC inner radii and buffer-IPyC gap is plotted in Fig. 4.7. Since the buffer-IPyC gap contributes a significant fraction of the thermal resistance in a TRISO particle, increasing gap size ought to result in increased temperature in the fuel kernel. This is verified in Fig. 4.8.

In earlier work at INL, a researcher computed the probability of IPyC layer cracking and SiC failure using PARFUME [39]. In those calculations, Abaqus was used to perform the FE stress analyses needed to capture the multidimensional effects of asphericity and IPyC cracking. In addition, a correlation function that includes higher-order terms was used to determine multidimensional stress. To make a commensurable comparison of BISON and PARFUME results, both codes should use the same linear stress correlation function and effective mean strength. 
Table 4.8: Fuel parameters.

\begin{tabular}{llc}
\hline Category & \multicolumn{1}{c}{ Parameter } & $\begin{array}{c}\text { Nominal values } \\
\text { Standard Deviation }\end{array}$ \\
\hline \multirow{5}{*}{ Fuel characteristics } & ${ }^{235}$ U enrichment $(\mathrm{wt} \%)$ & 15.5 \\
& Carbon/uranium (atomic ratio) & 0.4 \\
& Oxygen/uranium (atomic ratio) & 1.5 \\
\hline \multirow{5}{*}{ Particle geometry } & Kernel diameter $(\mu \mathrm{m})$ & $425 \pm 10$ \\
& Buffer thickness $(\mu \mathrm{m})$ & $100 \pm 15$ \\
& IPyC thickness $(\mu \mathrm{m})$ & $40 \pm 4$ \\
& SiC thickness $(\mu \mathrm{m})$ & $35 \pm 3$ \\
& OPyC thickness $(\mu \mathrm{m})$ & $40 \pm 4$ \\
\hline & Kernel density $\left(\mathrm{g} / \mathrm{cm}^{3}\right)$ & 11.0 \\
& Kernel theoretical density $\left(\mathrm{g} / \mathrm{cm}^{3}\right)$ & 11.4 \\
& Buffer density $\left(\mathrm{g} / \mathrm{cm}^{3}\right)$ & $1.05 \pm 0.1$ \\
& Buffer theoretical density $\left(\mathrm{g} / \mathrm{cm}^{3}\right)$ & 2.25 \\
& IPyC density $\left(\mathrm{g} / \mathrm{cm}^{3}\right)$ & $1.90 \pm 0.05$ \\
& OPyC density $\left(\mathrm{g} / \mathrm{cm}^{3}\right)$ & $1.90 \pm 0.05$ \\
& IPyC BAF & $1.045 \pm 0.005$ \\
& OPyC BAF & $1.035 \pm 0.005$ \\
\hline
\end{tabular}

The stress correlation factors and effective mean strength used in this comparison are precomputed for each compact by BISON, and their values are provided in Table 4.10. The probability of IPyC cracking and $\mathrm{SiC}$ failure was recomputed by PARFUME using the stress correlation factors and effective mean strength from BISON. Table 4.11 summarizes the comparison of results, showing good agreement between the two codes.

The evolution of $\mathrm{SiC}$ failure probability is shown in Fig. 4.9. The $\mathrm{SiC}$ failures of all three compacts in Capsule 5 are due to IPyC cracking, causing localized tensile stress in the $\mathrm{SiC}$ layer. Assuming 2,275 fuel particles per compact, the estimated number of failed particle is 1,2, and 1 in Compacts 5-1-3, 5-2-3, and 5-6-1, respectively. This is the same number of failed particles per compact that PARFUME predicted.

BISON's failure predictions compare very well to the results computed by PARFUME for AGR$5 / 6 / 7$. This provides evidence that the relevant equations were coded correctly and indicates that BISON is an appropriate simulation tool for TRISO failure analysis.

\subsection{Code Verification}

A formal proof that an analysis ran correctly is generally impossible because the expected code output cannot be precisely determined [40]. This is due to the fact that code results are depen- 


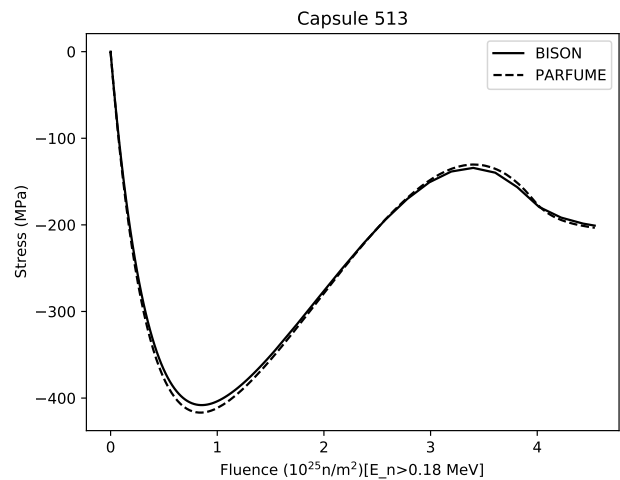

(a) Capsule 5-1-3.

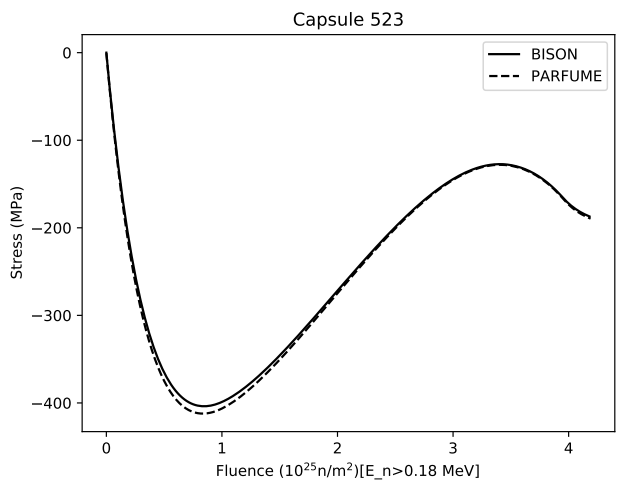

(b) Capsule 5-2-3.

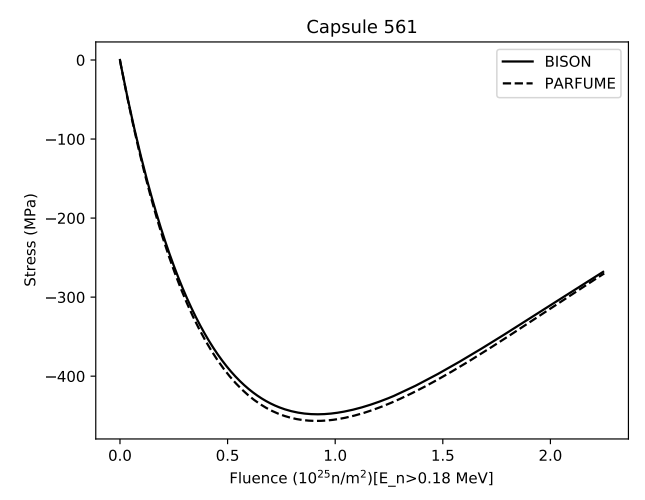

(c) Capsule 5-6-1.

Figure 4.6: Comparison of BISON and PARFUME results for inner-wall tangential stress in SiC. 


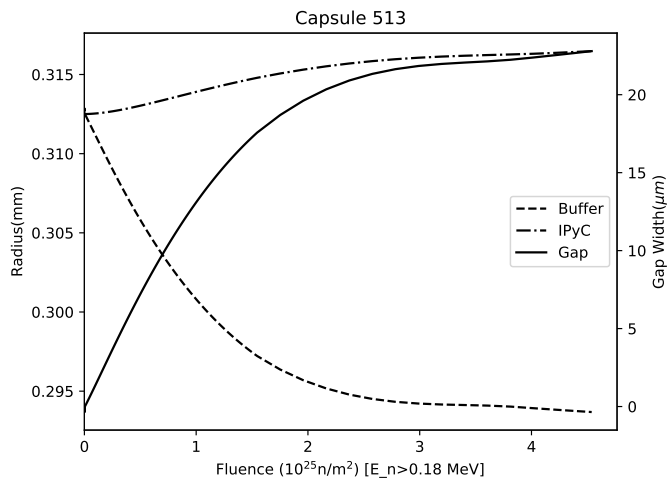

(a) Capsule 5-1-3.

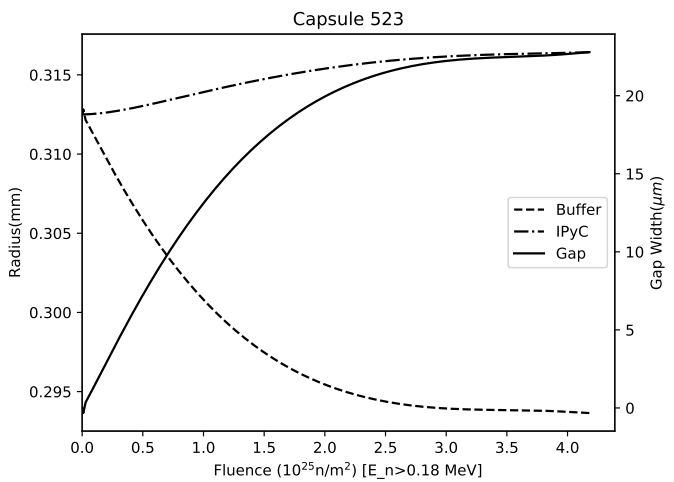

(b) Capsule 5-2-3.

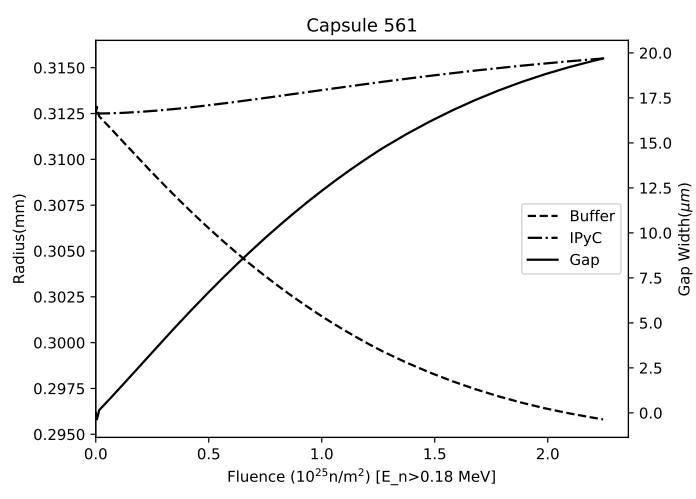

(c) Capsule 5-6-1.

Figure 4.7: Buffer outer and IPyC inner radii during irradiation. Gap is computed as their difference. 


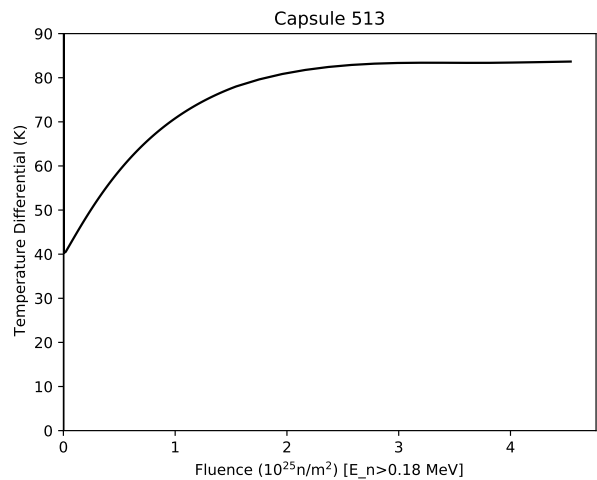

(a) Capsule 5-1-3.

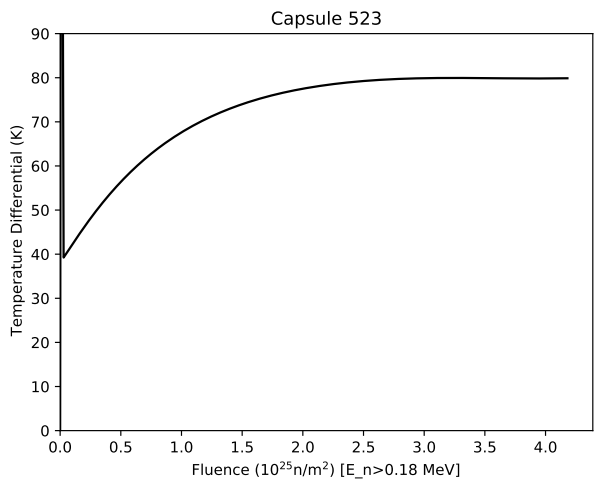

(b) Capsule 5-2-3.

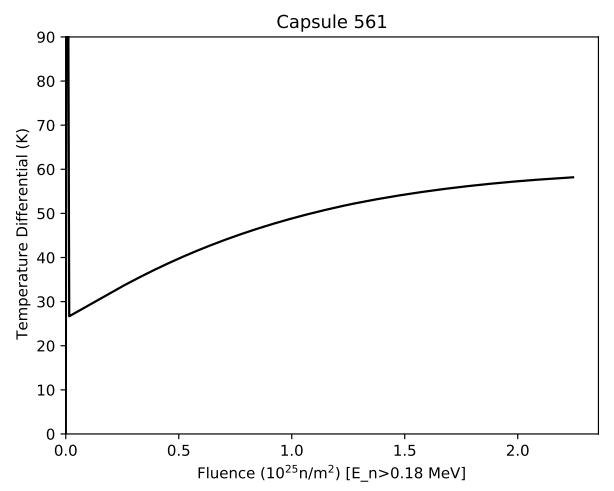

(c) Capsule 5-6-1.

Figure 4.8: Temperature from the kernel centerline to the outer surface of the OPyC . 


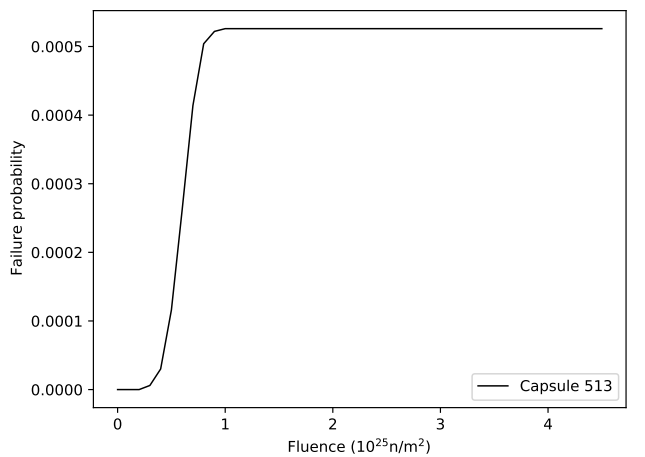

(a) Capsule 5-1-3.

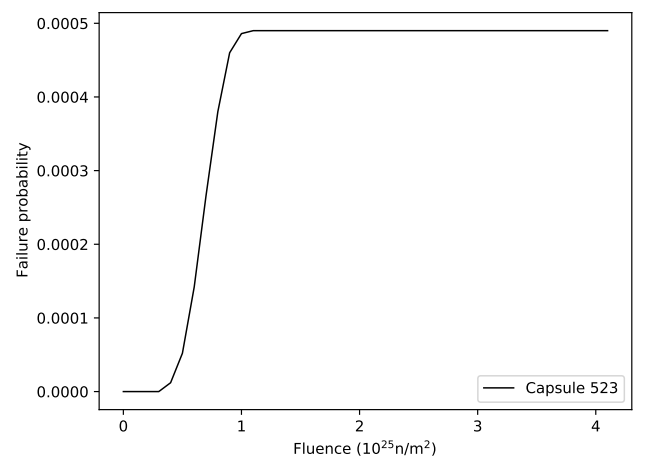

(b) Capsule 5-2-3.

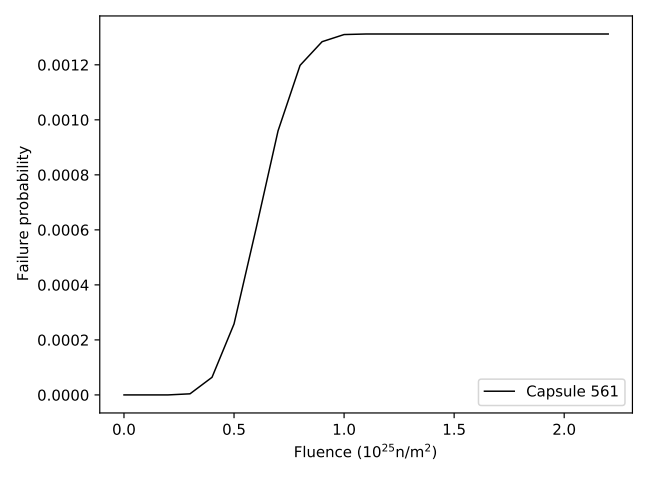

(c) Capsule 5-6-1.

Figure 4.9: Evolution of $\mathrm{SiC}$ failure probability. 
Table 4.9: Irradiation conditions.

\begin{tabular}{lcccc}
\hline Compact & EFPD & $\begin{array}{c}\text { Burnup } \\
(\% \text { FIMA })\end{array}$ & $\begin{array}{c}\text { Fast fluence } \\
\left(\times 10^{25} \mathbf{n} / \mathbf{m}^{2}\right)\end{array}$ & $\begin{array}{c}\text { Irradiation } \\
\text { temperature }\left({ }^{\circ} \mathbf{C}\right)\end{array}$ \\
\hline $5-1-3$ & 510 & 12.67 & 4.54 & 803 \\
$5-2-3$ & 510 & 12.17 & 4.19 & 812 \\
$5-6-1$ & 510 & 8.24 & 2.25 & 696 \\
\hline
\end{tabular}

Table 4.10: Stress correlation factors and effective mean strength.

\begin{tabular}{ccccccc}
\hline \multirow{2}{*}{ Compact } & Intact & \multicolumn{2}{c}{ IPyC cracking } & \multicolumn{3}{c}{ Asphericity } \\
\cline { 2 - 7 } & $\sigma_{\mathrm{ms}}(\mathrm{MPa})$ & $\sigma_{\mathrm{ms}}(\mathrm{MPa})$ & $-\frac{\bar{\sigma}_{2 \mathrm{D}}}{\bar{\sigma}_{1 \mathrm{D}}}$ & $\sigma_{\mathrm{ms}}(\mathrm{MPa})$ & $\frac{\bar{\sigma}_{2 \mathrm{D}}}{\bar{\sigma}_{1 \mathrm{D}}}$ & $\frac{\Delta \bar{\sigma}_{2 \mathrm{D}}}{\Delta \bar{\sigma}_{1 \mathrm{D}}}$ \\
\hline $5-1-3$ & 710 & 1199 & -0.96 & 988 & 1.53 & 1.33 \\
$5-2-3$ & 711 & 1199 & -0.96 & 998 & 1.53 & 1.34 \\
$5-6-1$ & 708 & 1199 & -0.96 & 1034 & 1.51 & 1.06 \\
\hline
\end{tabular}

Table 4.11: Failure probability.

\begin{tabular}{ccccc}
\hline \multirow{2}{*}{ Compact } & \multicolumn{2}{c}{ BISON } & \multicolumn{2}{c}{ PARFUME } \\
\cline { 2 - 5 } & IPyC cracking & SiC failure & IPyC cracking & SiC failure \\
\hline $5-1-3$ & $7.45 \times 10^{-1}$ & $7.34 \times 10^{-4}$ & $5.96 \times 10^{-1}$ & $5.88 \times 10^{-4}$ \\
$5-2-3$ & $6.55 \times 10^{-1}$ & $4.9 \times 10^{-4}$ & $5.62 \times 10^{-1}$ & $5.26 \times 10^{-4}$ \\
$5-6-1$ & $9.15 \times 10^{-1}$ & $1.31 \times 10^{-3}$ & $8.52 \times 10^{-1}$ & $1.33 \times 10^{-3}$ \\
\hline
\end{tabular}

dent upon many factors (e.g., the selected numerical algorithm, temporal/spatial discretization, iterative tolerances, machine precision, etc.). In the absence of a formal proof of correctness, code verification can instill confidence in the correctness of a scientific code. This is achieved through a quantified demonstration that the code results converge to a reference solution at the theoretical rate as the computational mesh and time discretization are refined.

Selection of a reference solution is the first task in the verification procedure. Here, we employ two methods for code verification:

1. The method of exact solutions (MES) [41] in which a known solution to the intended mathematical model is found as some nonlinear system operator $(\mathcal{L})$ (Eq. 4.1):

$$
\mathcal{L}[f(\vec{x}, t)]=0
$$

where the solution, $f(\vec{x}, t)$, is a function of space $(\vec{x})$ and time $(t)$.

Finding a non-trivial analytic solution to a complex nonlinear differential equation is dif- 
ficult. The solution to such equations often requires significant simplifying assumptions. For example, many analytic solutions require that one or more terms in the partial differential equation (PDE) be trivial and thus eliminated from the solution. This process becomes even more difficult when a system of nonlinear equations is considered.

2. The method of manufactured solutions (MMS) [40-43] in which we manufacture a particular form of the solution $M(\vec{x}, t)$, then seek the necessary source term, $Q(\vec{x}, t)$, that would result in the manufactured solution:

$$
\mathcal{L}[M(\vec{x}, t)]=Q(\vec{x}, t) .
$$

The source term $Q(\vec{x}, t)$ is implemented in the simulation tool, and then the verification process is performed. This methodology requires that the manufactured solution is formulated using continuous, smooth functions sufficiently complex to reveal nonlinearity in the governing equations. The chosen manufactured solution can be physically unrealistic, as it is intended only to test the underlying numerical algorithms.

Any necessary boundary conditions or initial conditions can be derived directly from the manufactured solution $M(\vec{x}, t)$. Any equations of state, varying properties, or nonlinear sources can be incorporated into the MMS process by implementing them in the nonlinear operator $\mathcal{L}$. This allows all relevant code options to be tested in different combinations. In addition, MMS does not require the complex analytic solutions formed for MES, greatly reducing the labor required for the verification process.

The theoretical rate of convergence (or the formal order of accuracy) can be determined through analysis of the linear truncation error (LTE) ${ }^{1}$. After we select the method to obtain solutions, the theoretical convergence rate of the numerical algorithm is established. Then, a numerical representation of the mathematical model is formulated and solved on at least three consecutive meshes. Global errors between the numerical solutions and the reference solution are calculated using error norms. For example, the $L_{2}$ norm (or Euclidean norm) of the error over the solution domain $\Omega$ is defined as:

$$
\|q\|=\left[\int_{\Omega}(q-\tilde{q})^{2} \mathrm{~d} \Omega\right]^{1 / 2}
$$

where the reference solution is represented by the primary variable $q$ (e.g., temperature for heat conduction, displacement for mechanics, etc.) and the numerical approximation is $\tilde{q}$.

The local LTE converges at some rate; therefore, the global error will converge at the same rate if the mesh size is (1) small enough to eliminate higher-order LTE terms and (2) large enough that numerical error is not dominant. In practical applications, this convergence behavior is characterized by three regions as shown in Fig. 4.10 [45]. To relate the error to the characteristic element size $h$, we use the following:

$$
\|q\|=C h^{\hat{p}}
$$

\footnotetext{
${ }^{1}$ The formal order of accuracy as derived for the finite element method can be found in [44].
} 
where $C$ is an arbitrary problem-dependent constant and $\hat{p}$ is the observed order of accuracy. Eq. 4.3 is in a power law form; therefore, the slope of the error on a log-log plot is equivalent to the observed order of accuracy $\hat{p}$ :

$$
\hat{p}=\frac{\log \left(\|q\|_{r h} /\|q\|_{h}\right)}{\log (r)}=\log _{r}\left(\frac{\|q\|_{r h}}{\|q\|_{h}}\right)
$$

where $\|q\|_{h}$ is the norm of $q$ at some mesh size $(h)$ and $r$ is the mesh refinement factor $(r \geq 2)$.

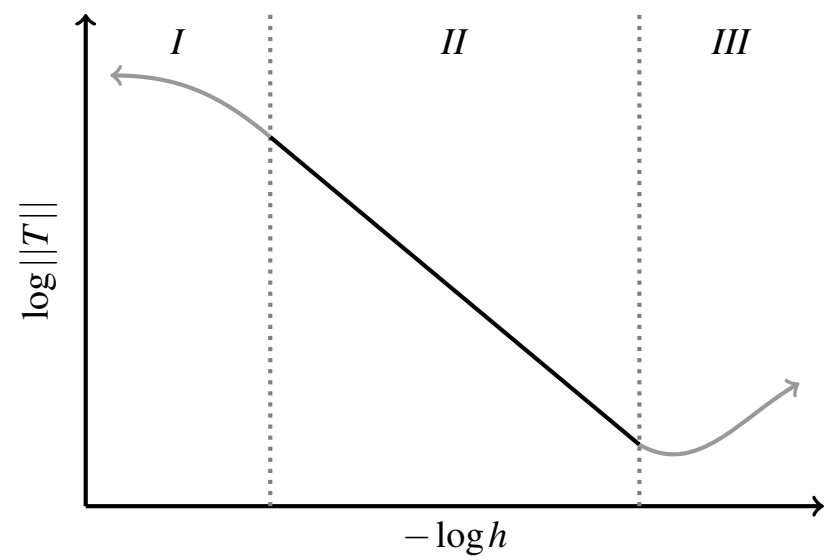

Figure 4.10: A pictorial representation of expected convergence behavior [44]. Region I represents coarse meshes, Region II is the asymptotic region, and Region III is caused by numerical error. The desirable region for the numerical solutions is the asymptotic region.

The code verification exercise is successful if the observed order of accuracy matches the formal order of accuracy. This provides confidence in the implemented numerical algorithm. Then, the problem is used as supporting evidence that the particular combination of physics, discretization, geometry, boundary conditions, and initial conditions is free of coding mistakes.

In this work, we demonstrate each method via a single problem: Problem 4.3.1 for the MES and Problem 4.3.2 for the MMS. Then, we examine many meshes to understand the convergence behavior.

Problem 4.3.1. A solid sphere has spatially dependent internal heating:

$$
q^{\prime \prime \prime}=q_{o}^{\prime \prime \prime}\left(1-\beta \frac{r^{2}}{r_{o}^{2}}\right)
$$

where the radius of the sphere is $r_{o}\left(0 \leq r \leq r_{o}\right)$ and $k$ is the constant thermal conductivity. It has a constant temperature imposed on its boundary: $T\left(r=r_{o}\right)=T_{o}$. The sphere reaches thermal equilibrium, and the analytic solution for the temperature distribution is [46, 47]:

$$
T(r)=T_{o}+\frac{q_{o}^{\prime \prime \prime} r_{o}^{2}}{6 k}\left[\left(1-\frac{r^{2}}{r_{o}^{2}}\right)-\frac{3 \beta}{10}\left(1-\frac{r^{4}}{r_{o}^{4}}\right)\right] .
$$


This problem is obtained from [44] and solved in BISON on the domain $\vec{X} \in[0,1]$. The center of the sphere uses a Neumann boundary condition (finiteness requirement), and the surface has a Dirichlet condition: $(d T / d r)_{r=0}=0$ and $T(1)=T_{R}=300 \mathrm{~K}$. Steady-state heat conduction is considered in the sphere with constant thermal conductivity $k=1 \mathrm{~W} / \mathrm{m} / \mathrm{K}$.

In Fig. 4.11a, the exact and computed solutions are shown for three different meshes and two FE types (first order, or linear, with two nodes per element; second order, or quadratic, with three nodes per element). A convergence study is conducted with a refinement factor of two $\left(r_{r}=2\right)$. The computed norms for each FE type and boundary condition type are plotted in Fig. 4.11b. Note that the $L_{2}$ norm quantifies convergence of the temperature distribution (solid lines) and the $H_{1}$ norm quantifies convergence of the heat flux (dashed lines). Slopes of first-, second-, and third-order convergence are indicated. The formal order of accuracy is two for linear FEs and three for quadratic FEs for the temperature solution and one less for the heat flux solution. In the asymptotic region for both cases, the linear and quadratic FE solutions converge to the exact solution with the correct order of accuracy.

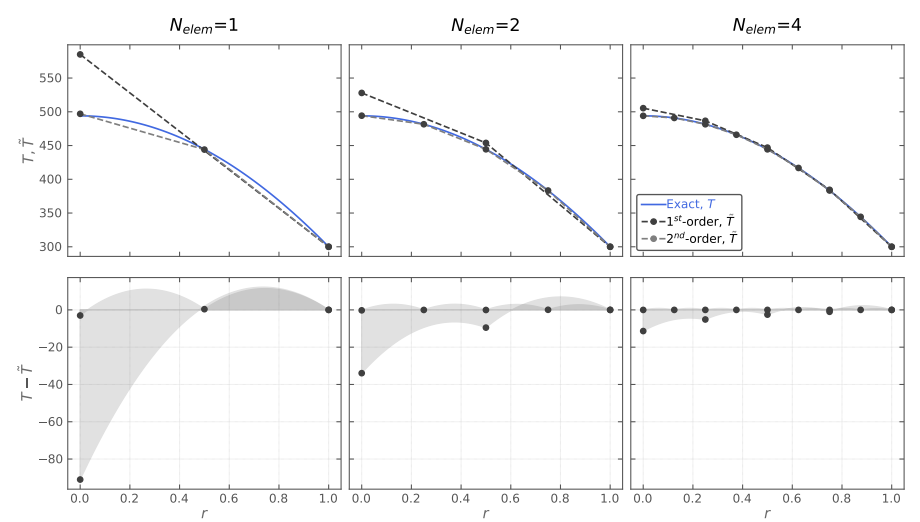

(a) Temperature distributions (first row) and residual (second row) for different meshes and finite elements.

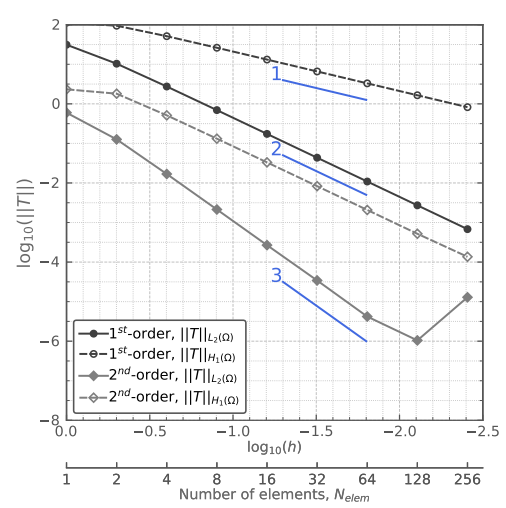

(b) Convergence plot $\left(r_{r}=2\right)$.

Figure 4.11: BISON results for Problem 4.3.1 using one-dimensional finite elements.

Problem 4.3.2. Fick's second law of diffusion follows the same mathematical model as the heat equation. A PDE with constant material properties can be written as:

$$
\frac{\partial u}{\partial t}=\kappa \nabla^{2} u
$$

where $u$ is temperature and $\kappa$ is thermal diffusivity for the heat conduction equation and $u$ is concentration and $\kappa$ is the diffusion coefficient for the diffusion equation.

Here, we analyze a steady-state one-dimensional heat conduction (or diffusion) equation using the manufactured solution:

$$
u(r)=\sin (a \pi r)
$$


This simple function is suitable for use in an MMS problem because it is continuous and infinitely differentiable. To find the source term $Q$ that produces the solution $u$, the steady-state heat conduction operator $\mathcal{L}=-\nabla \cdot \nabla$ is applied to $u$. This results in the following source term:

$$
Q=\mathcal{L}(u)=-\frac{2 a \pi}{r} \cos (a \pi r)+a^{2} \pi^{2} \sin (a \pi r) .
$$

The problem is solved with BISON on the domain $\vec{X} \in[0,1]$. The manufactured solution is shown in Fig. 4.12a using $a=2$, which is the value used in the BISON implementation. Steadystate heat conduction is considered through a homogeneous solid using the external source in Eq. 4.10. Dirichlet boundary conditions are derived from the manufactured solution using $a=2$.

The exact and computed solutions in Fig. 4.12 are shown for three different meshes and two FE types (first order, or linear, with two nodes per element; second order, or quadratic, with three nodes per element). A convergence study is conducted with a refinement factor of two $\left(r_{r}=2\right)$. The computed norms for each FE type and boundary condition type are plotted in Fig. 4.12b. Note that the $L_{2}$ norm quantifies convergence of the temperature distribution (solid lines) and the $H_{1}$ norm quantifies convergence of the heat flux (dashed lines). Slopes of first-, second-, and third-order convergence are indicated. The formal order of accuracy is two for linear FEs and three for quadratic FEs for the temperature solution and one less for the heat flux solution. In the asymptotic region for both cases, the linear and quadratic FE solutions converge to the exact solution with the correct order of accuracy.

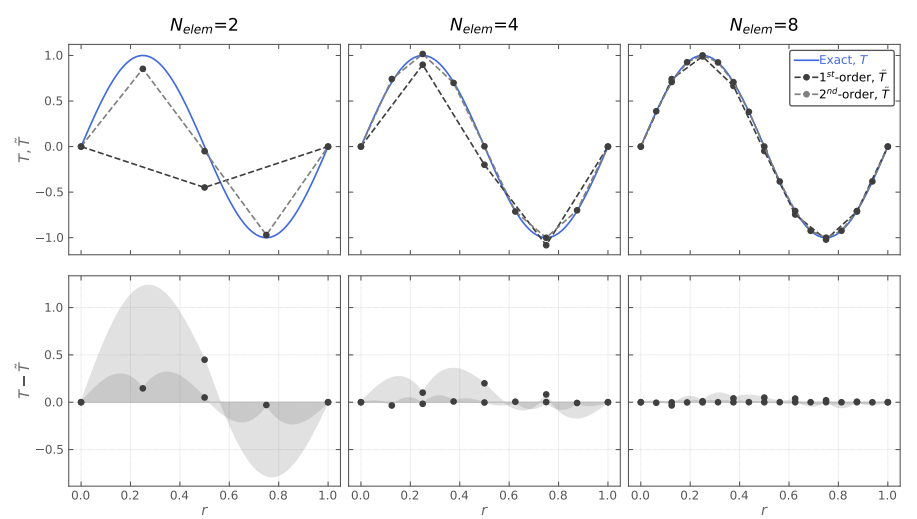

(a) Temperature distributions (first row) and residual (second row) for different meshes and finite elements.

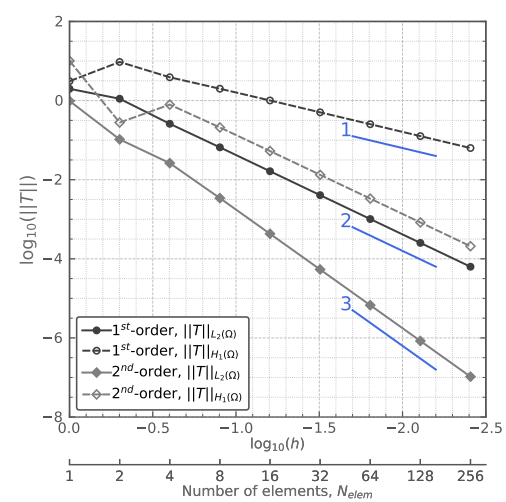

(b) Convergence plot $\left(r_{r}=2\right)$.

Figure 4.12: BISON results for the Problem 4.3.2 using one-dimensional finite elements.

These results show that the implementation of the heat equation (diffusion equation) with onedimensional finite elements is correct in BISON. For BISON results for similar verification problems, see [44]. 


\section{Validation}

Validation is the software development process for determining whether computed values match real-world conditions. It is the process of comparing code results to experimental data and is an essential part of determining whether a code is suitable for use as a design tool.

This chapter discusses fission product diffusion based on AGR-1 data.

\subsection{AGR-1}

DOE's Advanced Gas Reactor program sponsored a series of irradiation tests on TRISO particle fuel. The first of these, AGR-1, included post-irradiation examination (PIE) of the release of silver, cesium, and strontium. The details of AGR-1 are documented elsewhere (see [34] and the references therein), so only a brief orientation is given here.

The AGR-1 experiment was configured in capsules. Each capsule housed 12 fueled compacts as three stacks of four vertically aligned compacts. At six capsules, the experiment included a total of 72 compacts, each identified by a capsule number, vertical level within the capsule, and stack number. Irradiation conditions, including power level and compact-average temperature, are available for each day of irradiation.

The analysis procedure is as follows. Given that power and temperature are known only as average values at the compact level, a single particle is analyzed for each compact. The internal temperature of the particle depends on the power level, which provides an energy input due to fission in the fuel kernel, and the temperature at the outer surface of the particle, which is set to the compact average temperature. Fission product transport depends on fission yields (see Section 3.2.5.3), diffusion coefficients (see Section 3.2.3), and the computed temperature. At the outer surface of the particle, the mass passed outside the particle for any fission product is:

$$
r=\iint-D \nabla C \cdot n \mathrm{~d} t \mathrm{~d} A
$$

where $D$ is the Arrhenius diffusion coefficient for the given fission product (see Section 3.2.3), $C$ represents the concentration of the fission product, $n$ is the vector normal to the exterior surface, and $A$ is the outer surface of the particle.

The total fission product production is:

$$
p=\iint \Gamma \dot{F} \mathrm{~d} t \mathrm{~d} V
$$


where $\Gamma$ is the fission yield (atom/fission), $\dot{F}$ is the fission rate (fission/ $/ \mathrm{m}^{3}$-s), and $V$ represents the volume of the fuel kernel.

The release fraction is defined as $r / p$. The diffusion coefficient in the compact outside the particles is high enough to justify assuming that all fission products that left the particles will have left the compacts also.

The comparisons that follow for silver, cesium, and strontium are among the first fission product diffusion validation results using BISON for TRISO analysis. Other researchers have done similar work [48], but those results have not been independently checked and added to the BISON code repository.

\subsubsection{Silver Release}

To compare experimentally measured release fractions of silver, we follow the approach in [34]. In particular, we analyzed the 17 compacts listed in Table 3 of that work and plotted the results in a manner similar to Fig. 5 of that work. Our results, shown in Fig. 5.1, include values from PIE, PARFUME, and BISON. As seen from the figure, the BISON results compare very favorably with the PARFUME results.

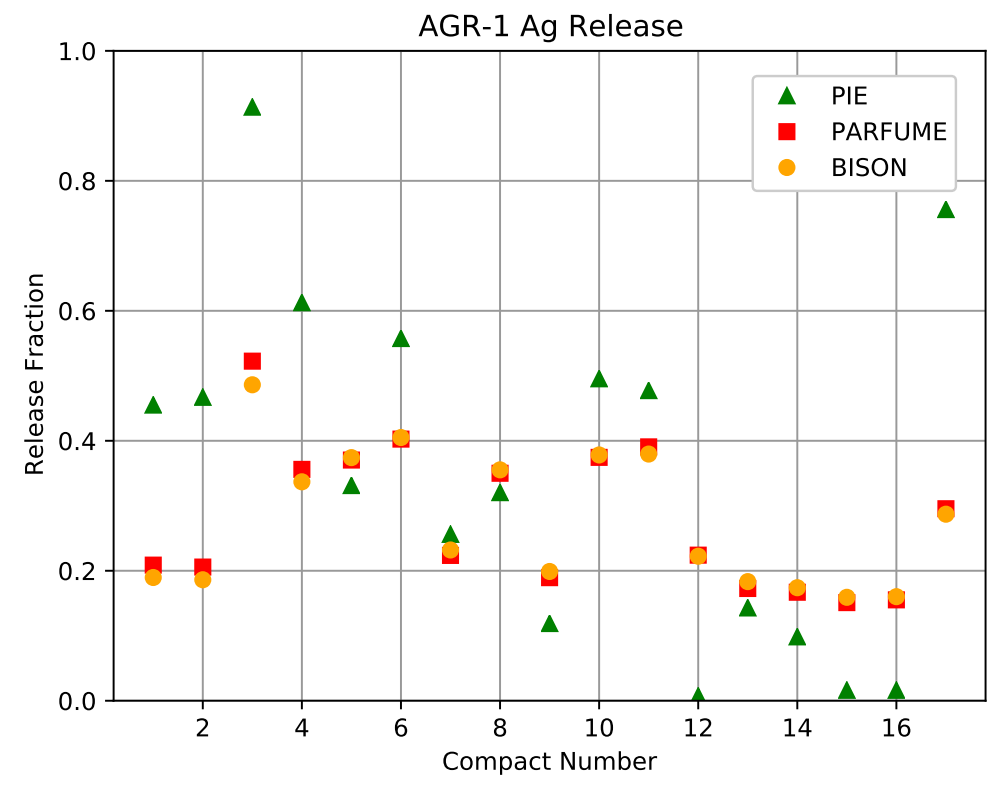

Figure 5.1: Comparison of measured and computed silver release fractions for seventeen compacts $(6-4-3,6-4-1,6-2-1,6-1-1,5-3-3,5-2-3,5-1-3,5-3-1,4-4-3,4-3-3,4-3-2$, 4-1-2, 4-4-1, 3-2-3, 3-3-1, 3-2-1, and 1-3-1).

Fig. 5.2 shows the concentration of silver in Compact 6-1-1 growing significantly over time. The sharp drop in concentration at about $0.8 \mathrm{r} / \mathrm{r}_{0}$ is due to the low diffusivity of silver in $\mathrm{SiC}$. 


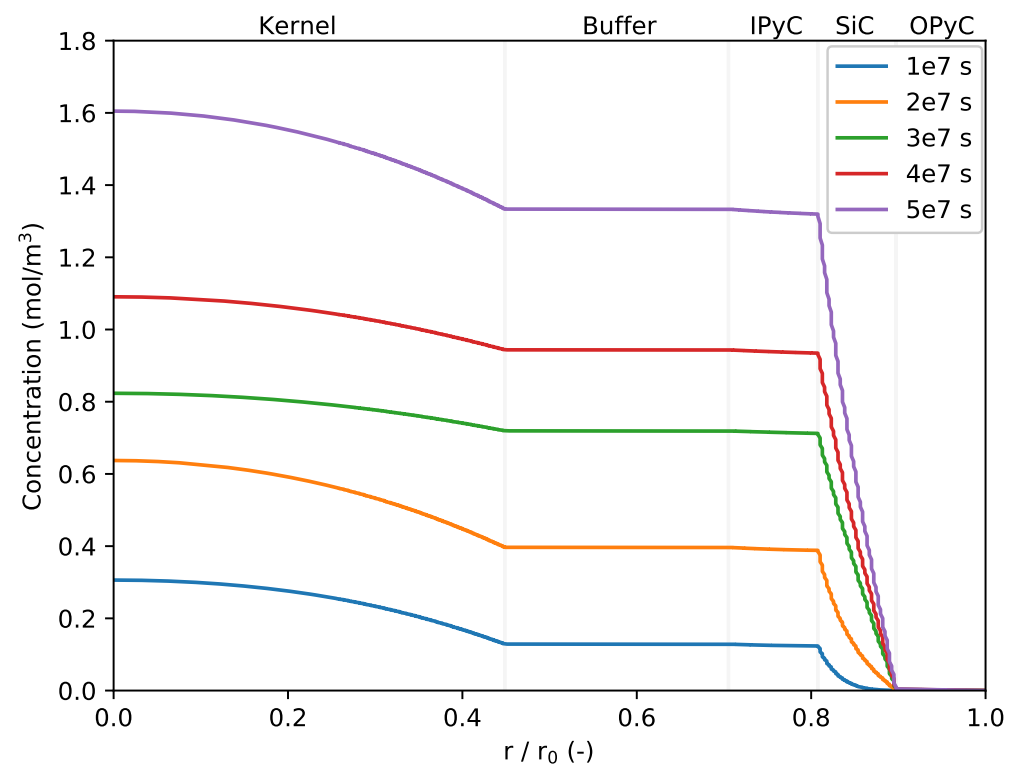

Figure 5.2: Concentration of silver in AGR-1 compact 6-1-1 over time. The abrupt drop in concentration is due to the low diffusivity of silver in $\mathrm{SiC}$.

\subsubsection{Cesium and Strontium Release}

The evaluation of cesium and strontium release mostly follows the same approach as for silver release. However, the comparisons for each fission product are made in two parts. In one set of comparisons, only compacts with no known particle failures are included. In the other comparisons, compacts with either one or two failed particles are included.

The analysis procedure for compacts with no known particle failures is exactly the same as that described for silver.

Two analyses were run for each compact with failed particles. The first was the standard analysis already described. In the second, which targeted failed particles, the fission product diffusivity of the $\mathrm{SiC}$ layer was set to a large value $\left(10^{-6} \mathrm{~m}^{2} / \mathrm{s}\right)$. The release fraction becomes:

$$
f=\frac{f_{i}\left(n-n_{f}\right)+f_{f} n_{f}}{n}
$$

where $f$ is the release fraction, $f_{i}$ is the release fraction from the intact particle, $f_{f}$ is the release fraction from the failed particle, $n$ is the total number of particles in the compact, and $n_{f}$ is the number of failed particles in the compact.

First, consider Fig. 5.3, which shows, for Compact 6-1-1, the concentrations of silver, cesium, and strontium at $5 \times 10^{7}$ seconds (approximately 580 days). The data for silver is the same as in Fig. 5.2. The concentrations of cesium and strontium are significantly higher than for silver. It is 
also worth noting that the concentration of strontium across the particle is qualitatively different than the concentration of cesium (and of silver, as seen in Fig. 5.2). Whereas the other two fission product concentrations drop dramatically at the $\mathrm{SiC}$ layer, the concentration of strontium drops dramatically at the interface between the kernel and the buffer. This is due to a very low diffusion coefficient for strontium in the kernel. Little strontium is released from the kernel.

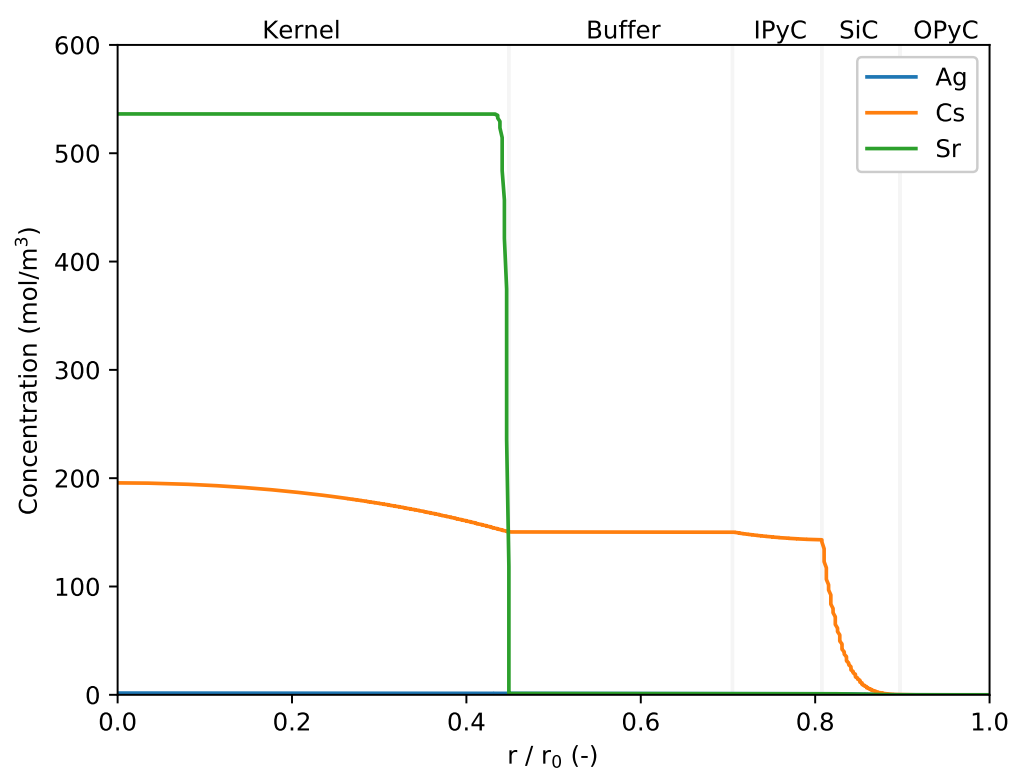

Figure 5.3: Concentration of silver, cesium, and strontium in Compact 6-1-1 at $5 \times 10^{7}$ seconds. The concentration of silver is better seen in Fig. 5.2.

The results for cesium release with intact particles are found in Fig. 5.4, and results for cesium release with failed particles are in Fig. 5.5. Aside from Compact 4-4-2, both PARFUME and BISON compute a higher release than seen in PIE for compacts with no failed particles (although for Compact 3-2-1, BISON's prediction nearly matches the maximum PIE value). Both PARFUME and BISON compute a higher release than seen in PIE for compacts with failed particles. Compared to PARFUME, BISON predicts slightly lower release for cesium with intact particles and slightly higher release for cesium with failed particles.

The results for strontium release with intact particles are in Fig. 5.6, and results for strontium release with failed particles are in Fig. 5.7. For compacts with intact particles, the PARFUME and BISON results are fairly consistent from compact to compact, while the PIE results show a large variation. These results show room for improvement, perhaps in the values of the diffusion coefficients. For compacts with failed particles, both PARFUME and BISON compute a higher release than seen in PIE. Compared to PARFUME, BISON predicts a slightly higher release for strontium, both with intact particles and with failed particles. In the case of strontium, it is interesting to note that predictions change only very slightly with the inclusion of failed particles. This is due to the fact that most of the strontium is held in the fuel kernel, never 


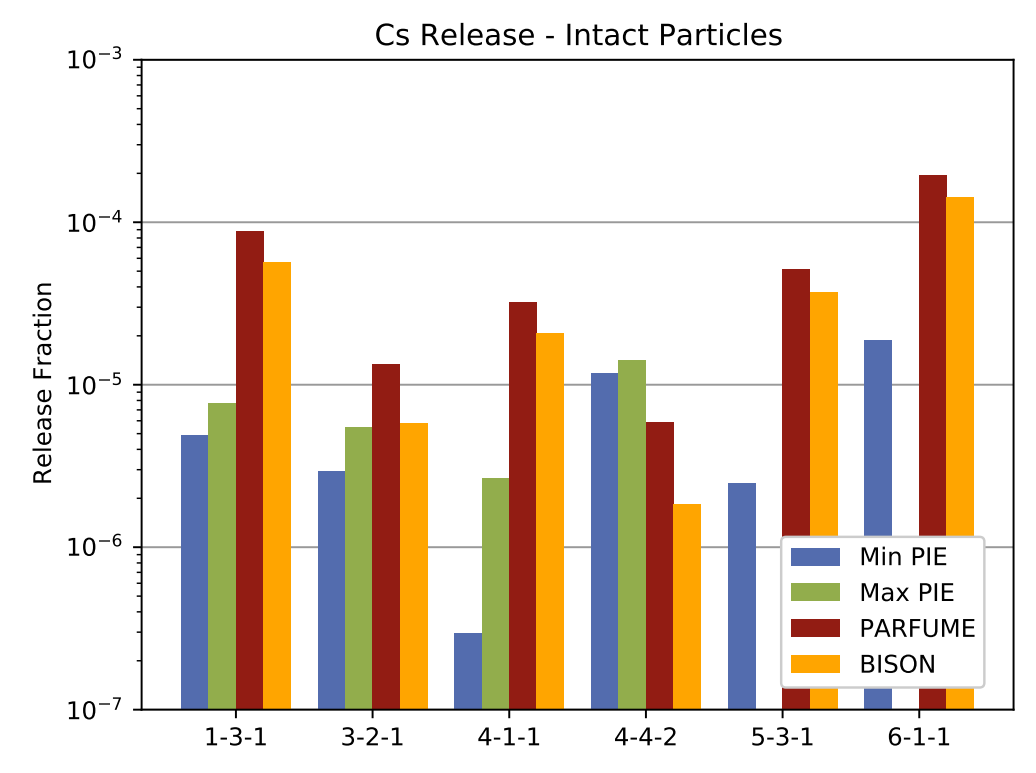

Figure 5.4: Comparison of measured and computed cesium release fractions for six compacts with no failed particles.

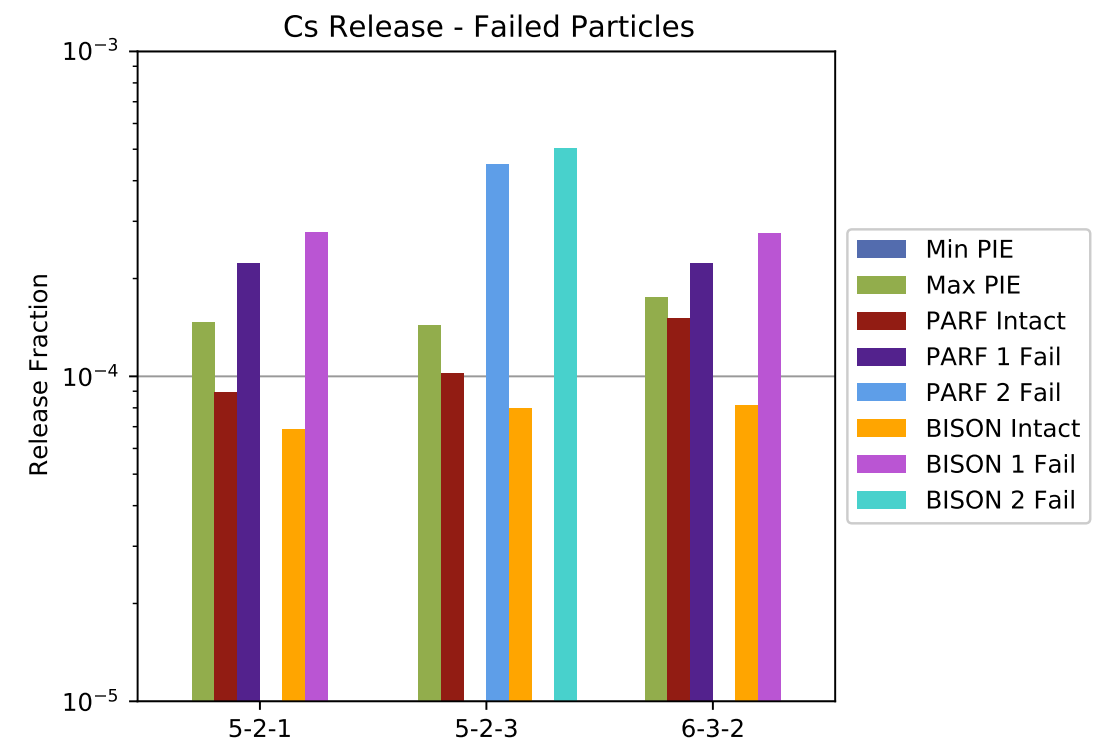

Figure 5.5: Comparison of measured and computed cesium release fractions for three compacts with one or two failed particles. 


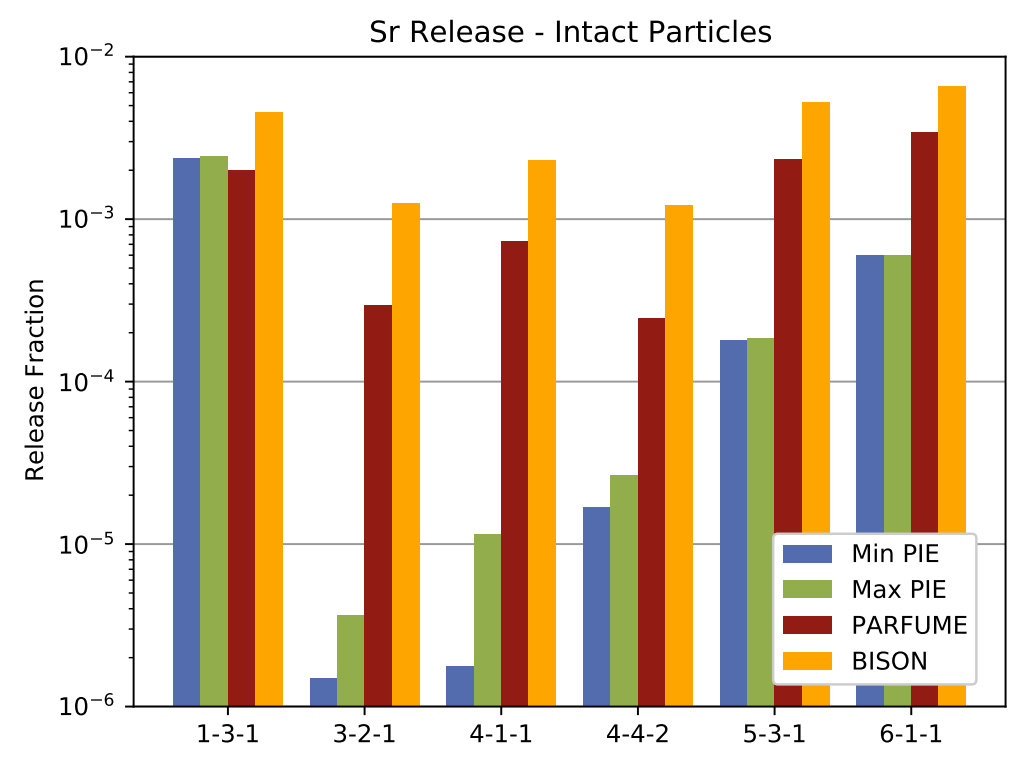

Figure 5.6: Comparison of measured and computed strontium release fractions for six compacts with no failed particles.

migrating outward to where a failed SiC layer would affect its release.

Differences in cesium and strontium diffusion between intact and failed particles can be seen in Fig. 5.8. This figure shows concentrations in Compact 6-3-2 at $5 \times 10^{7} \mathrm{~s}$ (approximately 580 days) for an intact particle and one in which the diffusion coefficient of the SiC layer was set to $10^{-6}$. For cesium in the intact case, the concentration drops dramatically across the $\mathrm{SiC}$ layer, as previously shown. For cesium in the case of a failed particle, the concentration hardly drops across the $\mathrm{SiC}$ layer (the dashed line is horizontal across the $\mathrm{SiC}$ layer). However, for strontium, the intact and failed results are indistinguishable. Small differences do exist but are not visible at this scale. The reason for this is that the kernel retains nearly all the strontium. The higher diffusion coefficient in the $\mathrm{SiC}$ layer has very little effect. Again, this effect is manifest in Fig. 5.7.

For silver, cesium, and strontium diffusion in AGR-1, BISON's computed results compare very favorably to PIE values and values computed by PARFUME. These results are evidence that BISON is an appropriate design tool for TRISO analysis. 


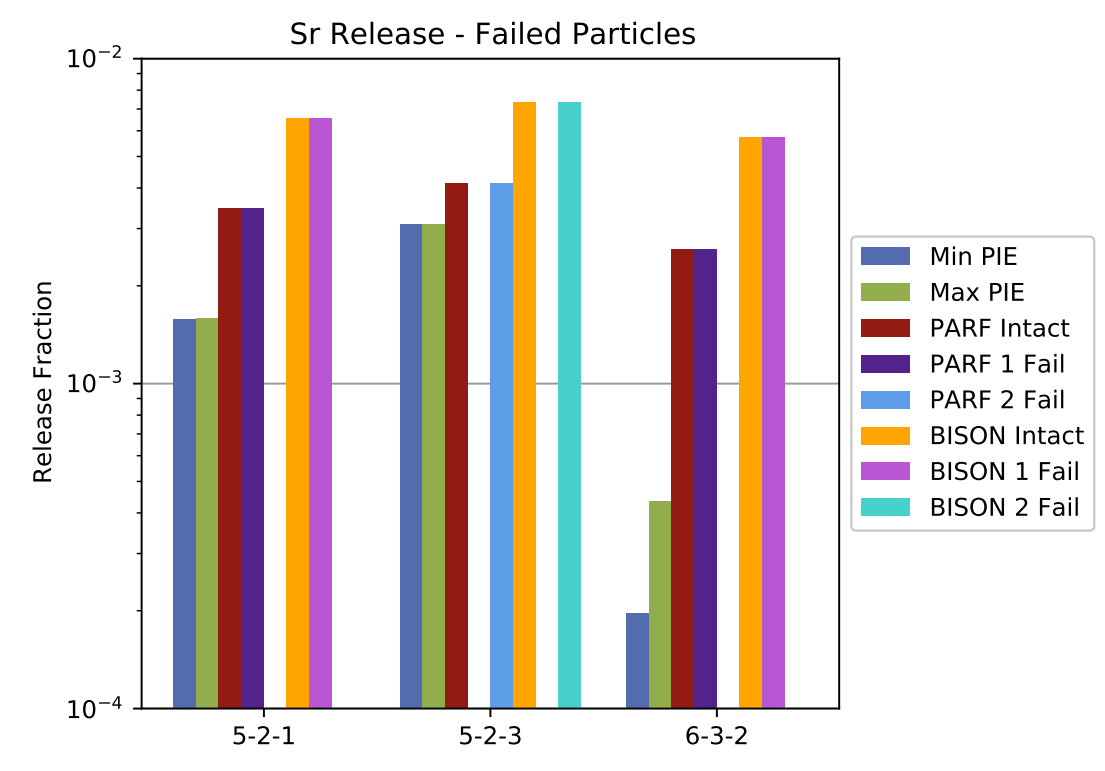

Figure 5.7: Comparison of measured and computed strontium release fractions for three compacts with one or two failed particles.

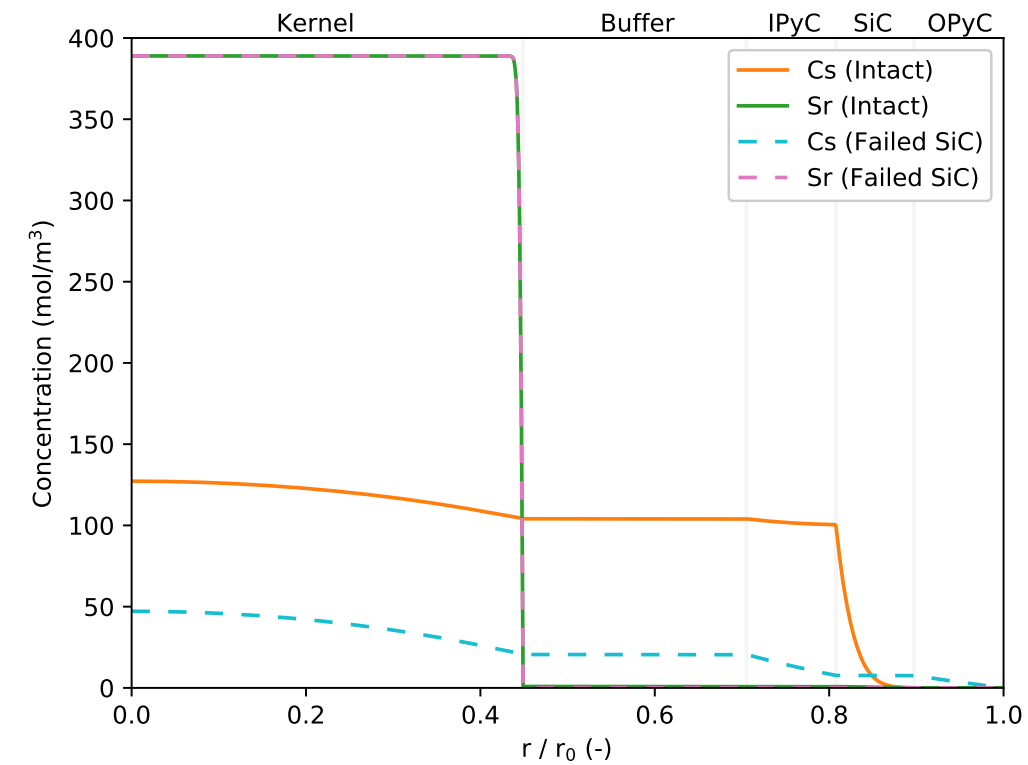

Figure 5.8: Concentrations of cesium and strontium for an intact and a failed particle in Compact 6-3-2. 


\section{Documentation and Training}

One advantage of MOOSE is its ability to integrate code, tests, and examples into web-based documentation. BISON takes advantage of this for its documentation (Fig. 6.1). The online documentation is freely available at https://mooseframework.org/bison and is updated periodically. Up-to-date documentation is available to those with an INL HPC account at https://hpcsc.inl.gov/ssl/BISON/site/. Finally, individual users on their local computers may build the documentation pages themselves. The input for the documentation is contained in the same code repository that houses the software and tests.

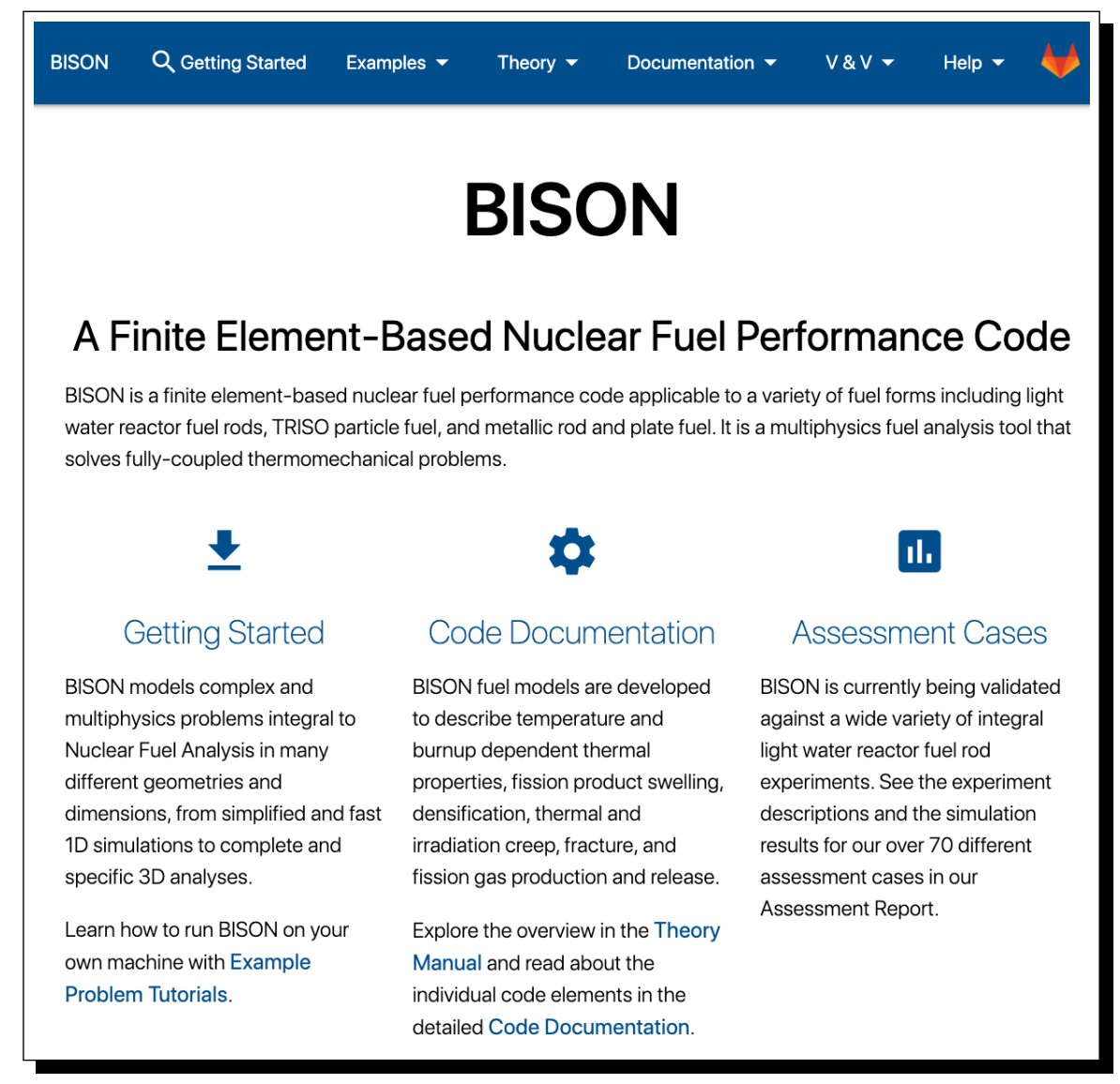

Figure 6.1: BISON homepage, https://mooseframework.org/bison. The most current version is available on INL's HPC. The documentation may also be built locally by individual users. 
The documentation includes a "Getting Started" section that walks users through the process of downloading BISON onto their computers and configuring it to run properly. The "Examples" section of the documentation shows working examples of input files for TRISO, metallic, mixed oxide, accident-tolerant LWR, and several traditional LWR fuel analyses. Other instructive material is also provided in that section.

The "Theory" section of the online documentation covers topics such as fundamental PDEs, equations of solid mechanics, nonlinear mechanical models, smeared cracking of ceramic fuels, and heat transfer across the fuel-cladding gap in LWRs.

The main content in the "Documentation" section is the BISON Specific Manual, a listing of all possible input options, with descriptions and examples linked to working input files. This is the main resource to help users understand BISON's input file. Also important are the links to workshop materials. These online workshop pages are the ones used when BISON training is presented. Finally, this section also includes information about software quality practices.

The "V\&V" section discusses verification and validation. The validation portion has dozens of working examples that users can run themselves.

\subsection{Tutorial on TRISO Analysis in BISON}

The BISON webpage includes a number of tutorials for new users. One of these focuses on TRISO fuel analysis (see Fig. 6.2). The tutorial discusses each of the major considerations when preparing a BISON input file for TRISO analysis. Most of these are associated with the materials in the particle. See, for example, Fig. 6.3. The tutorial includes a working input file that users can run and modify as they see fit (see Fig. 6.4).

\subsection{TRISO Workshop Material}

The BISON TRISO Workshop material includes an introduction to BISON and TRISO modeling, a review of code tests and benchmark cases, and a summary of major TRISO-related code features. It is intended to help users become familiar with the code so they can understand more in-depth documentation and begin to run their own TRISO analyses. Examples of the workshop material are in Fig. 6.5. This material is freely available at https: //mooseframework.org/bison_triso_workshop.

\subsection{Recent Training Workshops}

For several years, the BISON team has presented about two workshops each year to teach users how to run and modify the code. When requested, the team has also made special presentations 


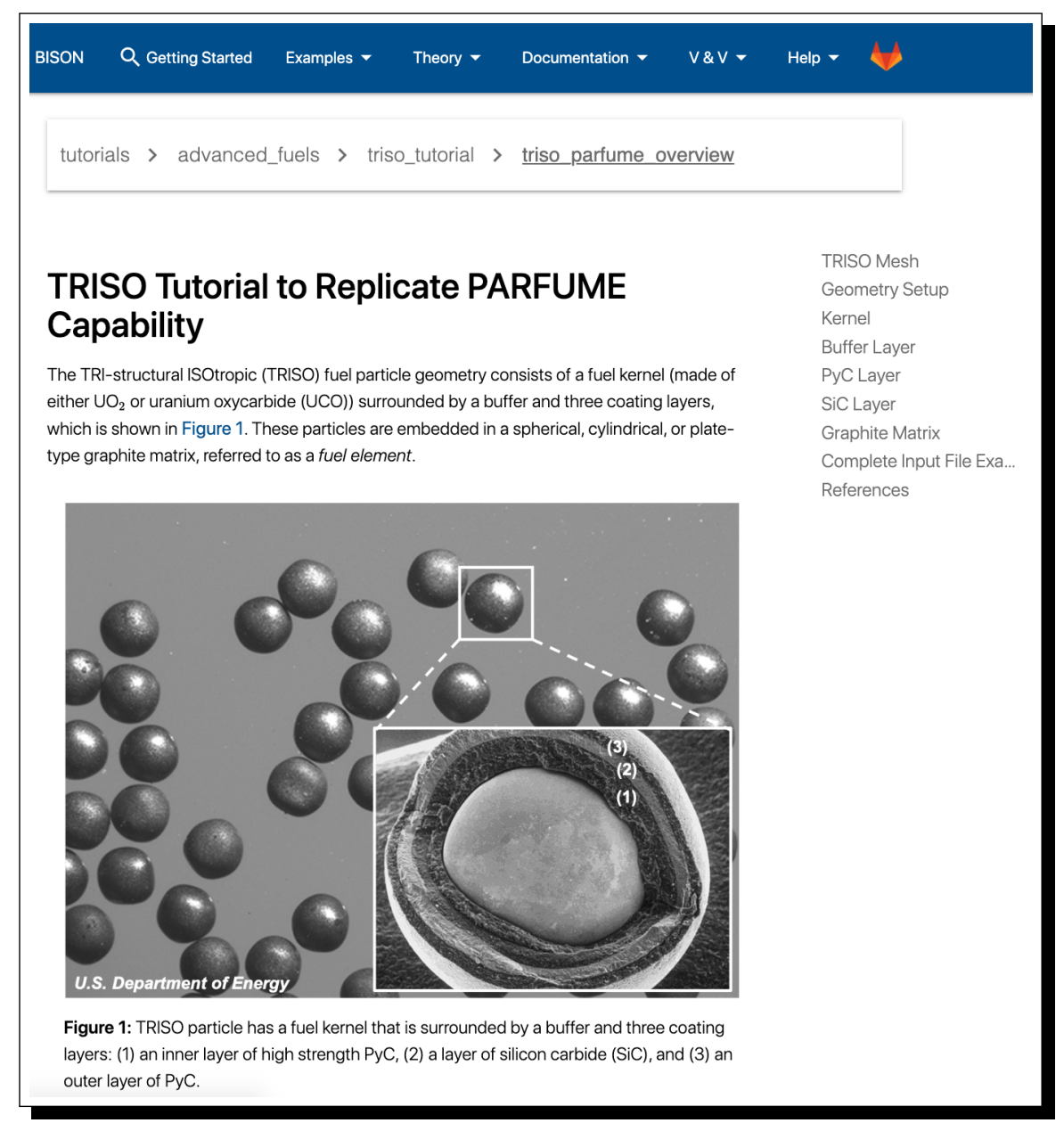

Figure 6.2: TRISO tutorial at https://mooseframework.org/bison/tutorials/ advanced_fuels/triso_tutorial/triso_parfume_overview.html.

to interested groups. In February 2020, two BISON team members traveled to Cranberry, Pennsylvania, to meet with Westinghouse Electric Company engineers and discuss TRISO modeling in BISON. This was the first time TRISO-specific BISON training was provided anywhere. Westinghouse engineers continue to explore BISON with the intention of using it for TRISO fuel performance analysis of their advanced reactor designs.

In June 2020, similar training was provided to NRC staff. Like Westinghouse, NRC is interested in BISON's TRISO capabilities. Due in part to Kairos's stated intention to use BISON, NRC wishes to become familiar with the physics and statistical modeling approaches in BISON. Due to travel restrictions, this training occurred remotely via teleconference software.

In workshops such as those mentioned, the BISON team uses materials described in this chapter. With these materials available online, those interested in BISON can explore the software at their own pace outside a classroom setting, and many more have access than would otherwise 


\begin{tabular}{|c|c|}
\hline $\begin{array}{l}\text { Kernel } \\
\text { Thermal and mechanical properties of } \mathrm{UCO} \text { are: } \\
\text { - Thermal properties } \\
\text { - Thermal expansion coefficient is set to } 10.0 \times 10^{-6}-/ \mathrm{K} \text { (value for } \mathrm{UO}_{2} \text { from Olander } \\
\text { (1976). } \\
\text { - Elastic properties } \\
\text { Burnup and fissiotion gas production and release models are: } \\
\text { - Burnup } \\
\text { - Fission gas release } \\
\text { Buffer Layer } \\
\text { Thermal and mechanical properties of the buffer are: } \\
\text { - Thermal properties } \\
\text { - Thermal expansion eigenstrain } \\
\text { - Clastic properties } \\
\text { - Irradiation-induced eigenstrain }\end{array}$ & $\begin{array}{l}\text { TRISO Mesh } \\
\text { Geometry Setup } \\
\text { | Kernel } \\
\text { Buffer Layer } \\
\text { PyC Layer } \\
\text { SiC Layer } \\
\text { Graphite Matrix } \\
\text { Complete Input File Exa... } \\
\text { References }\end{array}$ \\
\hline
\end{tabular}

Figure 6.3: Discussion of the kernel and buffer layer at the TRISO tutorial page at https://mooseframework.org/bison/tutorials/advanced_fuels/triso_ tutorial/triso_parfume_overview.html.

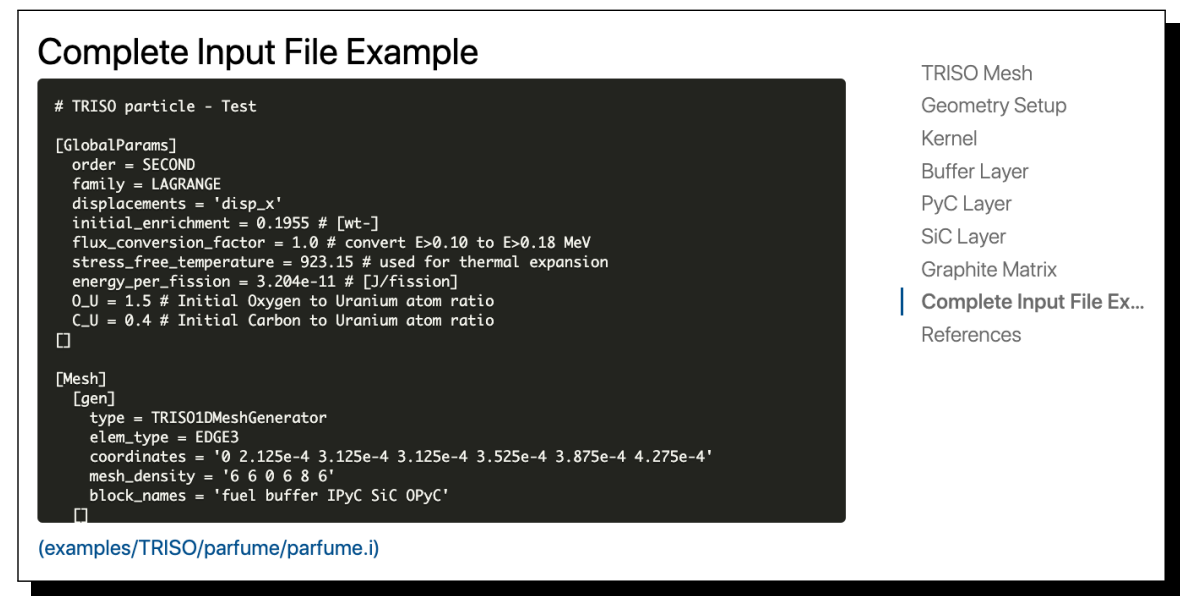

Figure 6.4: A working input file is part of the TRISO tutorial page at https: //mooseframework.org/bison/tutorials/advanced_fuels/triso_ tutorial/triso_parfume_overview.html.

be possible. Having the training materials linked to the source code and tests in the repository makes them much easier to maintain and improve. 
Recent TRISO Development A more complete set of models from PARFUME

- Elastic properties $\quad$ PyC

$\begin{array}{ll}- \text { Elastic properties } & \cdot \text { Pyc } \\ \text { - Thermal properties } & - \text { Creep }\end{array}$

- Mass diffusion properties - Irradiation strain $\begin{array}{lll}- \text { Mass diffusion properties } \quad \text { Kernel } & \text { Sic }\end{array}$

- Swelling

- Fission

- Buffer
- Creep

- Irradiation strai

Sic

- Palladium penetration

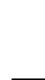

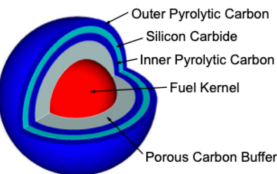

Asphericity

Stress correlation function: $\sigma_{c}=\frac{\bar{\partial} 2 D}{\bar{\sigma}_{1 D}-\min }+\frac{\Delta \bar{\sigma}_{D D} D}{\Delta \sigma_{1 D}} \Delta \sigma_{1 D}$
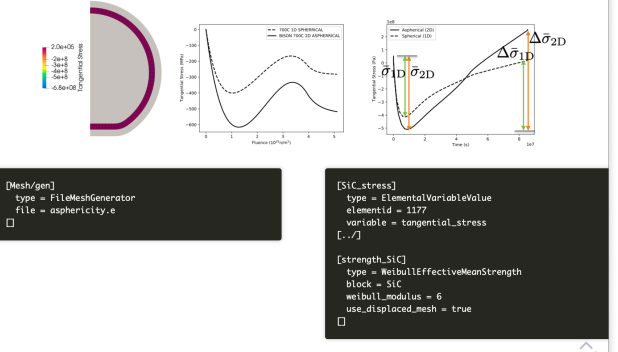

Mesh Generation

- Clearly mesh generation depends on the type of the analysis to be run - 1D (fast, spherically symmetric) 2D (medium, axisymmetric)

(STlow symmetry across planes or not symmetric)
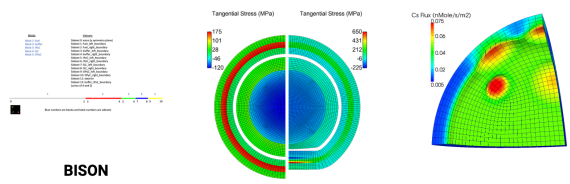

BISON/CUBIT

CUBIT

TRISO Failure modes

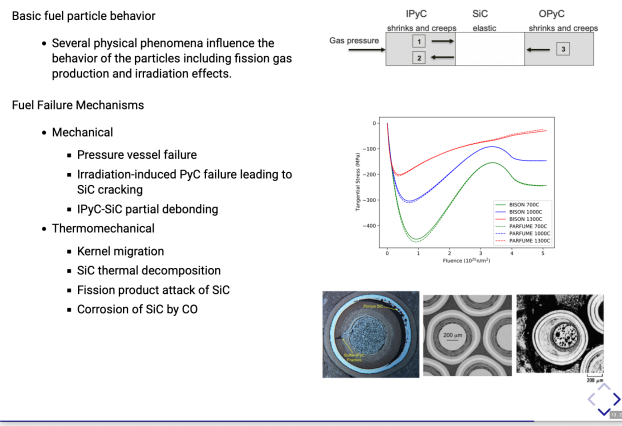

Figure 6.5: Representative slides from the TRISO workshop at https://mooseframework. org/bison_triso_workshop. 


\section{Conclusions and Future Work}

While the finite-element nuclear fuel performance code BISON has had a TRISO analysis capability for several years, only in the last couple of years has the advanced reactor community shown interest in the code. This increased interest has spurred increased TRISO development through NEAMS and collaborations with private industry.

One of the most significant events regarding TRISO capabilities in BISON is the development of a close partnership between the BISON team and Kairos Power. Connections through NEAMS and a successful U.S. DOE Funding Opportunity Announcement application have enabled work that is clearly in Kairos Power's interest while also advancing capabilities applicable to the industry at large. Kairos Power has been instrumental in guiding new development, providing initial code implementations and documentation for new features, reviewing proposed software, and helping INL staff better understand TRISO fuel.

Significant advances in BISON's capabilities have been made. BISON is able to build a onedimensional or two-dimensional TRISO mesh automatically. This dramatically speeds up analysis and enables automated sensitivity and parameter exploration studies.

Many new material models are available for the fuel kernel, buffer, pyrolytic carbon, $\mathrm{SiC}$, and matrix. These models include mechanical (elastic, creep, irradiation strain), thermal (thermal conductivity, specific heat), mass diffusion, and fission gas production and release models.

Equally important is the development of a new capability for statistical analysis of TRISO fuel particle failure. This capability is essential for modeling the thousands of particles in a reactor, only a very few of which will fail. This capability relies on Weibull analysis, two-dimensional and three-dimensional analysis of failure modes, the ability to tie those higher-dimensional failure modes to fast-running one-dimensional analyses, and parallel computing.

Major advances were also made in ensuring that solutions computed by BISON are correct. Ten benchmark cases for fuel performance from the IAEA CRP-6 originally run with BISON several years ago were re-run. The remaining three cases were run for the first time. In addition, several diffusion cases from CRP-6 were run for the first time. In all cases, results either matched analytic solutions or, where no analytic solution exists, were comparable to results computed from other codes. In addition, a rigorous verification exercise was completed in which BISON was shown to converge to the correct analytic solution upon mesh refinement for a set of nontrivial problems.

BISON results have been validated against experimentally obtained fission product diffusion data. The AGR-1 experiment, in which a number of capsules loaded with TRISO particles were irradiated and fission product diffusion measured, provides data on the movement of silver, 
cesium, and strontium. For all three species, the BISON results compare favorably with the experimental data and with results computed by PARFUME. This represents a milestone in the development of BISON, demonstrating that BISON results match real-world conditions.

Finally, a significant effort has been completed to make BISON's documentation more widely and readily available. Documentation on material and other models, a tutorial, and online workshop slides are freely available to anyone through https://moose framework.org/bison, and a set of example and benchmarking problems are included in the BISON repository. Training was provided in FY20 to Westinghouse and NRC staff, with follow-on discussions anticipated. Others who have shown interest include NASA, BWXT, X-energy, and General Atomics. The close collaboration with Kairos Power was emphasized in this report.

While a great deal has been accomplished-moving BISON's TRISO capability from being a preliminary demonstration to handling statistical failure and being validated on fission product diffusion data-much remains to be done. The understanding of fission product diffusion requires significant investigation, in no small part due to the fact that diffusion is often not the mechanism by which fission products transverse a fuel particle. In many cases, movement is accelerated by chemical changes, grain boundaries, or cracks. These phenomena are not understood well and are not modeled at present. Modeling these mechanisms will require close coordination with lower-length-scale researchers. Another area that needs further development is improving the statistical failure capability by adding support for more failure modes, such as chemical attack on the $\mathrm{SiC}$ layer. Finally, more validation work is needed. In particular, the Advanced Gas Reactor (AGR) test series includes much more data, and BISON's ability to predict the response of TRISO fuel will be checked against that data. 


\section{Bibliography}

[1] IAEA, "Advances in high temperature gas cooled reactor fuel technology," Tech. Rep. IAEA-TECDOC-1674, International Atomic Energy Agency, 2012.

[2] R. Gontard and H. Nabielek, "Performance Evaluation of Modern HTR TRISO Fuels," Report HTA-1B-05/90, Forschungszentrums Juelich, July 1990.

[3] U. Littmark and J. F. Ziegler, "Handbook of Range Distributions for Energetic Ions in All Elements," tech. rep., Pergamon Press, 1980.

[4] C. J. Permann, D. R. Gaston, D. Andrš, R. W. Carlsen, F. Kong, A. D. Lindsay, J. M. Miller, J. W. Peterson, A. E. Slaughter, R. H. Stogner, and R. C. Martineau, "MOOSE: Enabling massively parallel multiphysics simulation,” SoftwareX, vol. 11, p. 100430, 2020.

[5] K. J. Geelhood and W. G. Luscher, "FRAPCON-3.5: A computer code for the calculation of steady-state, thermal-mechanical behavior of oxide fuel rods for high burnup," Tech. Rep. NUREG/CR-7022, PNNL-19418 Vol.1, Rev. 1, Pacific Northwest National Laboratory, 2014.

[6] K. J. Geelhood, W. G. Luscher, and J. M. Cuta, "FRAPTRAN-1.5: A computer code for the transient analysis of oxide fuel rods," Tech. Rep. NUREG/CR-7023 Vol.1, PNNL-19400 Vol.1, Pacific Northwest National Laboratory, 2014.

[7] "Fuel Analysis and Licensing Code : FALCON MOD01- Volume 1: Theoretical and Numerical Bases," Tech. Rep. EPRI - 1011307, Electric Power Research Institute, Palo Alto, CA, USA, December 2004.

[8] K. Lassmann, "TRANSURANUS: a fuel rod analysis code ready for use," Journal of $\mathrm{Nu}$ clear Materials, vol. 188, pp. 295-302, 1992.

[9] A. N. Laboratory, "LIFE/LIFEMETAL Fuels Performance Analysis Codes." http://www.osti.gov/bridge/servlets/purl/885496-PjEy9G/885496.PDF.

[10] T. Kobayashi, M. Kinoshita, S. Hattori, T. Ogawa, Y. Tsuboi, M. Ishida, S. Ogawa, and H. Saito, "Development of the sesame metallic fuel performance code," Nuclear Technology, vol. 89, no. 2, pp. 183-193, 1990.

[11] W. Hwang, C. Nam, T. S. Byun, and Y. C. Kim, "Macsis: A metallic fuel performance analysis code for simulating in-reactor behavior under steady-state conditions," Nuclear Technology, vol. 123, no. 2, pp. 130-141, 1998. 
[12] G. K. Miller, D. A. Petti, J. T. Maki, D. L. Knudsen, and W. F. Skerjanc, "PARFUME theory and model basis report," Tech. Rep. INL/EXT-08-14497, Idaho National Laboratory, September 2009.

[13] J. Wang, An Integrated Performance Model for High Temperature Gas Cooled Reactor Coated Particle. PhD thesis, Massachuseets Institute of Technology, 2004.

[14] D. G. Martin, "Considerations pertaining to the achievement of high burn-ups in HTR fuel," Nuclear Engineering and Design, vol. 213, pp. 241-258, 2002.

[15] M. Phelip, F. Michel, M. Pelletier, G. Degeneve, and P. Guillermier, "The ATLAS HTR fuel simulation code objectives, description and first results," in 2nd International Topical Meeting on High Temperature Reactor Technology, (Beijing, China), pp. 1-10, 2004.

[16] R. L. Williamson, J. D. Hales, S. R. Novascone, M. R. Tonks, D. R. Gaston, C. J. Permann, D. Andrs, and R. C. Martineau, "Multidimensional multiphysics simulation of nuclear fuel behavior," Journal of Nuclear Materials, vol. 423, pp. 149-163, 2012.

[17] Abaqus, Abaqus 6.9 Documentation. Dassault Systèmes, 2009.

[18] D. Petti, P. Martin, M. Phelip, and R. Ballinger, "Development of improved models and designs for coated-particle gas reactor fuels," Tech. Rep. INL/EXT-05-02615, Idaho National Engineering and Environmental Laboratory, December 2004.

[19] J. D. Hales, R. L. Williamson, S. R. Novascone, D. M. Perez, B. W. Spencer, and G. Pastore, "Multidimensional multiphysics simulation of TRISO particle fuel," Journal of $\mathrm{Nu}$ clear Materials, vol. 443, pp. 531-543, November 2013.

[20] Sandia National Laboratories, "CUBIT: Geometry and mesh generation toolkit." http://cubit.sandia.gov, 2008.

[21] H. Nabielek, K. Fukuda, K. Minato, and T. Ogawa, "Calculation of Particle Temperatures in NSRR Tests," unpublished draft, Japanese Atomic Energy Agency, March 1992.

[22] J. K. Fink, "Thermophysical properties of uranium dioxide," Journal of Nuclear Materials, vol. 279, pp. 1-18, 2000.

[23] G. K. Miller, D. A. Petti, J. T. Maki, D. L. Knudson, , and W. F. Skerjanc, "PARFUME Theory and Model Basis Report," Report INL/EXT-08-14497 (Rev.1), Idaho National Laboratory, September 2018.

[24] F. Ho, "NP-MHTGR: Material Models of Pyrocarbon and Pyrolytic Silicon Carbide," Report CEGA-002820 Rev. 1, CEGA Corporation, July 1993.

[25] V. Barabash, I. Mazul, R. Latypov, A. Pokrovsky, and C. H. Wu, "The effect of low temperature neutron irradiation and annealing on the thermal conductivity of advanced carbonbased materials," Journal of Nuclear Materials, vol. 307-311, pp. 1300-1304, 2002. 
[26] L. L. Snead, T. Nozawa, Y. Katoh, T.-S. Byun, S. Kondo, and D. A. Petti, "Handbook of sic properties for fuel performance modeling," Journal of Nuclear Materials, vol. 371, pp. 329-377, 2007.

[27] J. G. Stone, R. Schleicher, C. P. Deck, G. M. Jacobsen, H. E. Khalifa, and C. A. Back, "Stress analysis and probabilistic assessment of multi-layer sic-based accident tolerant nuclear fuel cladding," Journal of Nuclear Materials, vol. 466, pp. 682-697, 2015.

[28] C. Folsom, C. Xing, C. Jenson, H. Ban, and D. Marshall, "Experimental measurement and numerical modeling of the effective thermal conductivity of TRISO fuel compacts," Journal of Nuclear Materials, vol. 458, pp. 198-205, 2015.

[29] IAEA, "Heat Transport and Afterheat Removal for Gas Cooled Reactors Under Accident Conditions," Tech. Rep. TECDOC-1163, International Atomic Energy Agency, 2000.

[30] D. R. Olander, Fundamental aspects of nuclear reactor fuel elements. Technical Information Center, Energy Research and Development Administration, 1976.

[31] J. J. Powers and B. D. Wirth, "A review of TRISO fuel performance models," J Nuclear Materials, vol. 405, no. 1, pp. 74-82, 2010.

[32] K. Verfondern, H. Nabielek, M. J. Kania, and H.-J. Allelein, "High-Quality Thorium TRISO Fuel Performance in HTGRs," tech. rep., Forschungszentrums Juelich, 2013.

[33] T. Burchell, "AGC-1 Irradiation Creep Strain Data Analysis," Report ORNL/TM2014/255, Oak Ridge National Laboratory, September 2014.

[34] B. P. Collin, D. A. Petti, P. A. Demkowicz, and J. T. Maki, "Comparison of silver, cesium, and strontium release predictions using parfume with results from the agr-1 irradiation experiment," Journal of Nuclear Materials, vol. 466, pp. 426 - 442, 2015.

[35] "Fuel performance and fission product behavior in gas cooled reactors," Tech. Rep. IAEATECDOC-978, IAEA, 1997.

[36] A. H. Booth, "A method of calculating gas diffusion from $\mathrm{UO}_{2}$ fuel and its application to the X-2-f loop test," Tech. Rep. AECL-496, Atomic Energy of Canada Ltd., 1957.

[37] J. A. Turnbull, C. A. Friskney, J. R. Findlay, F. A. Johnson, and A. J. Walter, "The diffusion coefficients of gaseous and volatile species during the irradiation of uranium dioxide," Journal of Nuclear Materials, vol. 107, pp. 168-184, 1982.

[38] B. P.Colin, "Agr-5/6/7 irradiation experiment test plan,” Tech. Rep. INL/MIS-17-43095, Idaho National Laboratory, September 2017.

[39] W. F. Skerjanc, "AGR-5/6/7 irradiation test predictions using PARFUME," Tech. Rep. INL/EXT-17-43189, Idaho National Laboratory, September 2017.

[40] P. J. Roache, Verification and Validation in Computational Science and Engineering. Albuquerque, NM: Hermosa Publishing, 1998. 
[41] K. Salari and P. Knupp, "Code verification by the method of manufactured solutions," Tech. Rep. SAND2000-1444, Sandia National Laboratories, June 2000.

[42] W. L. Oberkampf and C. J. Roy, Verification and Validation in Scientific Computing. Cambridge, UK: Cambridge University Press, first ed., November 2010.

[43] M. P. McHale et al., "Standard for verification and validation in computational fluid dynamics and heat transfer," Standard ASME V\&V 20-2009, American Society of Mechanical Engineers, 2009.

[44] A. Toptan, N. W. Porter, J. D. Hales, R. L. Williamson, and M. Pilch, "FY20 verification of BISON using analytic and manufactured solutions," Tech. Rep. CASL-U-2020-1939-000; SAND2020-3887R, CASL, March 2020.

[45] D. S. Burnett, Finite Element Analysis from Concepts to Applications. Reading, MA: Addison-Wesley Publishing Company, 1987.

[46] J. H. VanSant, “Conduction heat transfer solutions," Tech. Rep. UCRL—52863-Rev.l; DE87 012387, Lawrence Livermore National Lab., August 1983.

[47] R. B. Bird, W. E. Stewart, and E. N. Lightfoot, Transport Phenomena. New York, New York: John Wiley \& Sons, Inc., 1960.

[48] D. Schappel, K. Terrani, L. Snead, and B. Wirth, "Modeling radionuclide release of triso bearing fuel compacts during post-irradiation annealing tests," Nuclear Engineering and Design, vol. 357, p. 110428, 2020. 